\title{
Modeling A 300 kHz Bathymetric Sonar System
}

by

Kenneth Alan Malmquist

B.S., Drexel University, Philadelphia (1985)

Submitted in partial fulfillment of the

requirements for the degree of

MASTER OF SCIENCE IN OCEANOGRAPHIC ENGINEERING

at the

MASSACHUSETTS INSTITUTE OF TECHNOLOGY

and the

WOODS HOLE OCEANOGRAPHIC INSTITUTION

September 1992

(c) Kenneth Alan Malmquist 1992

The author hereby grants to MIT and WHOI permission to reproduce

and distribute copies of this thesis document in whole or part.

Signature of Author

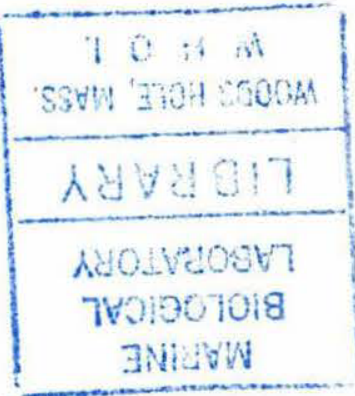

Joint Program in Oceanographic Engineering

Massachusetts Institue of Technology/

Woods Hole Oceanographic Institution

September 1992

Certified by

W. Kenneth Stewart, Jr., PhD.

Woods Hole Oceanographic Institution

Thesis Supervisor

Accepted by

Àrthur B. Baggeroer, $\mathrm{PhD}$.

Chairman, Joint Committee for Applied Ocean Science and Engineering

Massachusetts Institute of Technology/

Woods Hole Oceanographic Institution

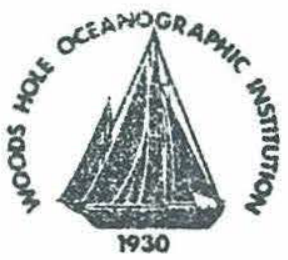




\title{
Modeling A 300 kHz Bathymetric Sonar System
}

\author{
by \\ KENNETH ALAN MALMQUIST
}
Submitted to the Massachusetts Institute of Technology/
Woods Hole Oceanographic Institution
Joint Program in Oceanographic Engineering
on September 1, 1992 in partial fulfillment of the
requirements for the degree of

MASTER OF SCIENCE IN OCEANOGRAPHIC ENGINEERING

\begin{abstract}
The Deep Submergence Laboratory has developed a family of calibrated high frequency bathymetric sonar systems for underwater survey. It is useful to have a detailed mathematical description of these systems to assist in data processing.

A model of a generalized sonar system is developed first. This model then is made specific to the DSL $300-\mathrm{kHz}$ forward scanning sonar and is implemented using the MATLAB software package. The model consists of a cascaded series of filters representing the electrical and mechanical components of the system. The model is adjusted after comparison to the transmitted pulse. The results are then inverted to demonstrate how the corrupting effects of the system can be reversed. A technique is developed for applying this reverse model to actual data.
\end{abstract}

The results showed that a good representation of the system can be implemented using relatively simple descriptions of each component. The most important components are the band-limiting filter and the transducer. It is possible to reverse model these components with good results.

Thesis Supervisor: Dr. W. Kenneth Stewart

Associate Scientist

Woods Hole Oceanographic Institution 


\section{Acknowledgements}

This thesis was made possible by the generous support provided by my friends and colleagues at the Deep Submergence Laboratory. In particular, I would like to thank my advisor, W. Kenneth Stewart, for his thoughtful commentary, feedback and patience. Steve Lerner was very helpful and accommodating while I conducted experiments, for which I am grateful. Hanu Singh and Bob Elder gave their time generously in answering my questions. John Kusters donated his time and computer equipment in helping with the production.

I would like to give special thanks to Skip Kolve of the University of Washington's Applied Physics Laboratory for his patience and generosity while answering my questions during numerous telephone conversations. His valuable insights and suggestions made this work possible.

The United States Navy is gratefully acknowledged for the graduate education opportunity provided. 


\section{Author's Biographical Note}

LT Kenneth A. Malmquist, USN, completed his undergraduate studies at Drexel University, where he received a Bachelor of Science in Physics in 1985. Following commission in the U.S. Navy, he completed nuclear propulsion and submarine training in 1987. LT Malmquist was assigned to the USS GUARDFISH (SSN-612), where he completed Submarine Warfare qualification in 1989. On GUARDFISH he served as Electrical Officer, Reactor Controls Assistant, Chemistry and Radiological Controls Assistant, and Communications Officer. He is presently assigned to the Massachusetts Institute of Technology/ Woods Hole Oceanographic Institution Joint Program in Oceanographic Engineering.

LT Malmquist's next assignment, following completion of his graduate studies, will be the Submarine Officers Advanced Course in Groton, Connecticut. Following this school, he will be assigned as a Department Head aboard a U.S. Navy submarine. 


\section{Contents}

\section{Chapter 1. Introduction}

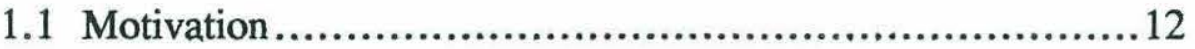

1.2 Research Objectives.............................................13

1.3 Outline Of Thesis..................................................13

Chapter 2. Background On Sidescan Sonar

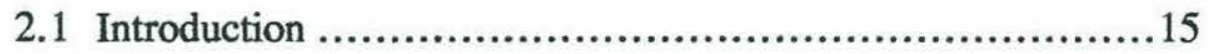

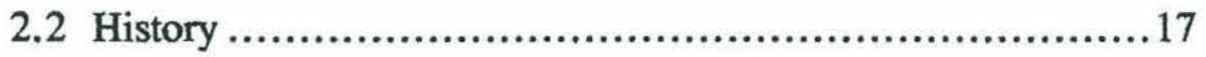

2.3 Modeling....................................................... 19

Chapter 3. Generalized Model Of A Sonar System

3.1 Introduction .....................................................23

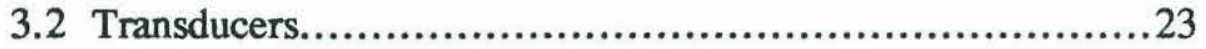

3.3 Pulse Generation.................................................27

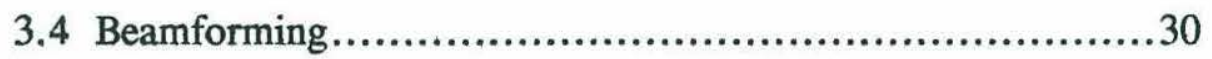

3.5 Receivers .................................................... 32

3.6 Post Processing ................................................. 34

Chapter 4 The DSL Bathymetric Sonar System

4.1 Introduction ........................................................ 37

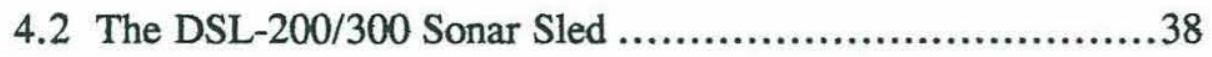

4.3 DSL $300 \mathrm{kHz}$ Sonar Operation...............................40 
Chapter 5 The DSL $300 \mathrm{kHz}$ Sonar Model

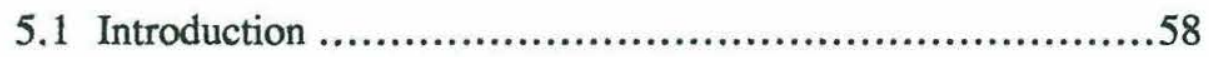

5.2 The Transmitter Section ........................................61

5.3 The Receiver Section ...................................... 78

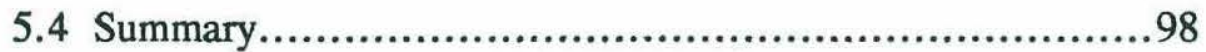

\section{Chapter 6 Inverse Modeling}

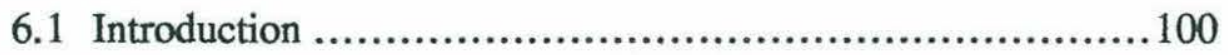

6.2 The Transducer ................................................. 101

6.3 The Bandpass Filter ........................................... 107

6.4 The Phase-Compensating Filters .............................110

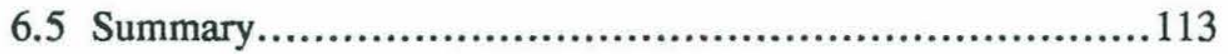

Chapter 7 Summary, Conclusions, and Recommendations

Appendix - MATLAB Macros For DSL-300 Model 


\section{Figures}

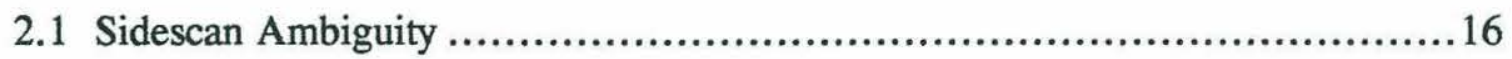

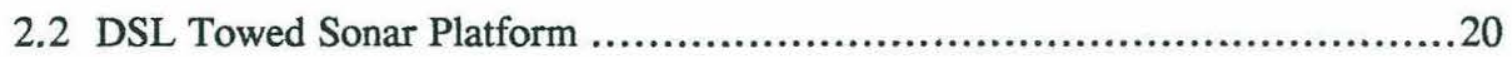

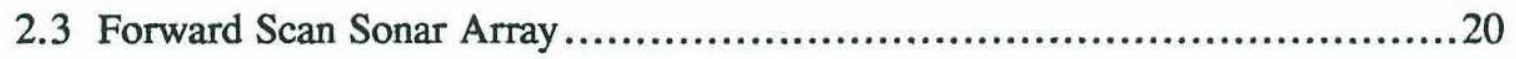

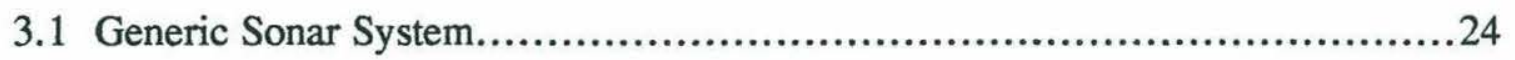

3.2 Mechanical Oscillator And Electrical Analog ......................................25

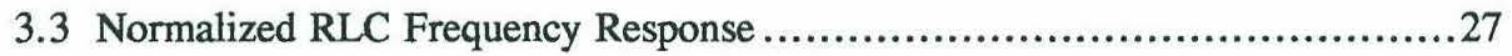

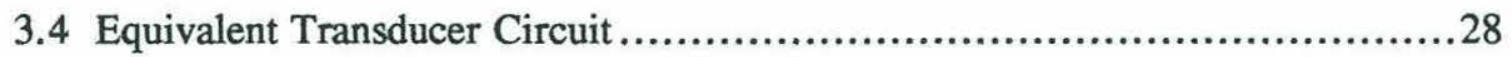

3.5 Finite Linear Array ......................................................................

3.6 Fourier Transform Of Square Signal ....................................................3

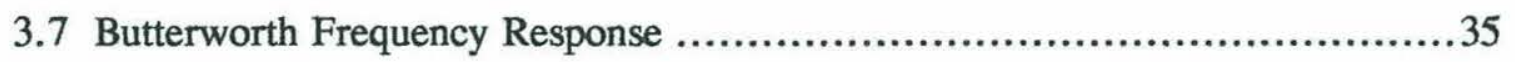

4.1 DSL Calibrated Bathymetric Sonar System............................................

4.2 DSL-200/300 Towed Sonar Platform........................................................ 39

4.3 DSL-200/300 Block Diagram............................................................... 40

4.4 Class B Type Amplifier...................................................................

4.5 Transmit/Receive Network..............................................................43

4.6 Measured Transmit Response Of Transducer............................................45

4.7 Measured Receive Response Of Transducer ...........................................47

4.8 Vertical Beam Pattern........................................................................ 48

4.9 Horizontal Beam Pattern................................................................... 49

4.10 Angle Ambiguity …................................................................... 49

4.11 Non-Inverting And Inverting Amplifier Configurations...........................50

4.12 Ideal Sample-And-Hold Amplifier.......................................................53

4.13 Intentional Undersampling Without Aliasing ….....................................55

4.14 Quadrature Downsampling Scheme ……...........................................56

5.1 DSL-300 Model Flow Chart ............................................................... 


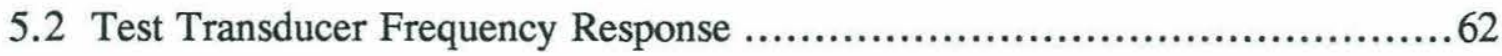

5.3 Experimental Setup For Determining Pulse Characteristics.....................62

5.4 Transmitted Pulse Envelope...................................................... 63

5.5 Model Transmitted Pulse Before Transducer ......................................65

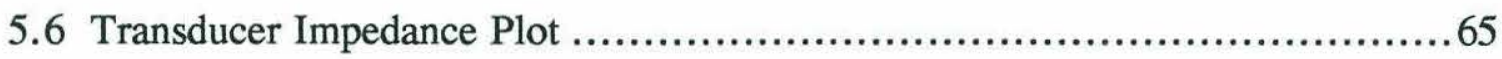

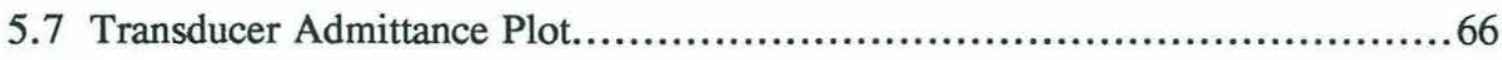

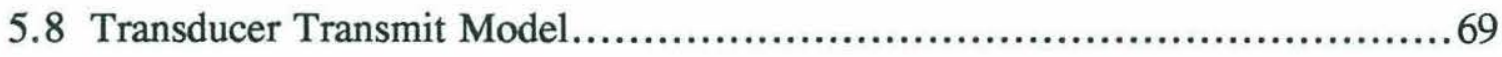

5.9 Transmitting Transducer Frequency Response …..............................71

5.10 Transmitting Transducer Phase Response .....................................71

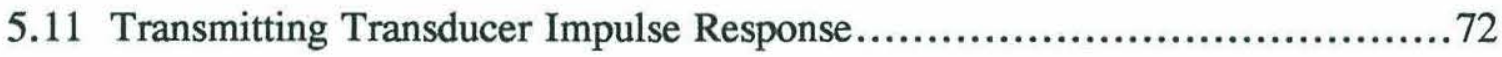

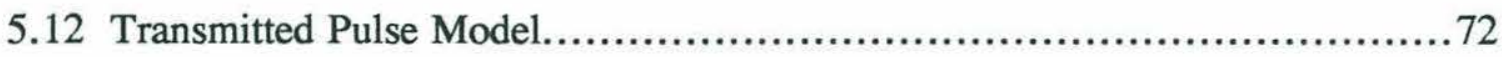

5.13 Transmitted Pulse Power.......................................................... 73

5.14 Modified Transmitted Pulse .................................................... 74

5.15 Normalized Spectrum Of Transmitted Pulse ................................... 75

5.16 Filter To Compensate For Test Hydrophone ................................... 76

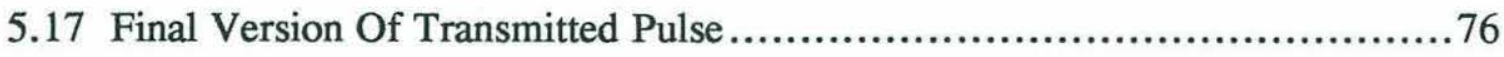

5.18 Final Transmitted Pulse Power................................................... 77

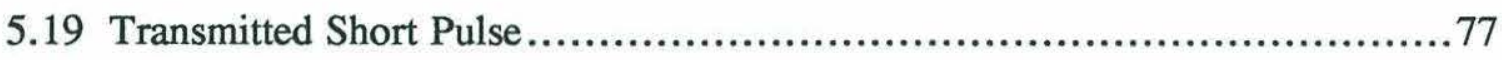

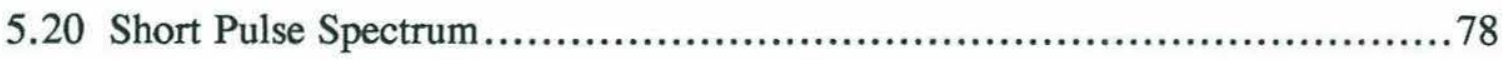

5.21 Receiving Transducer Equivalent Circuit........................................79

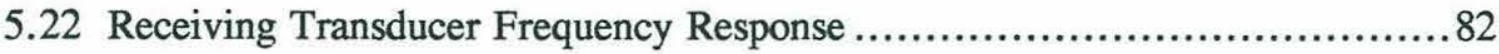

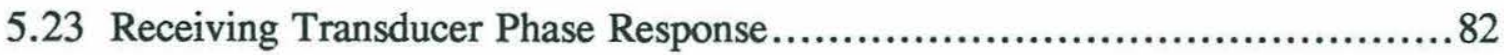

5.24 Receiving Transducer Impulse Response ..................................... 83

5.25 Returned Pulse At First-Stage Input............................................. 84

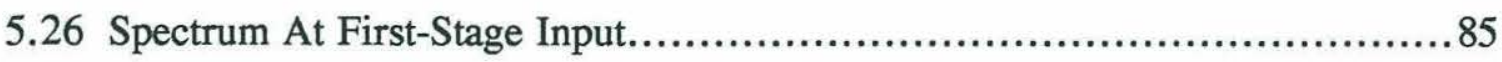

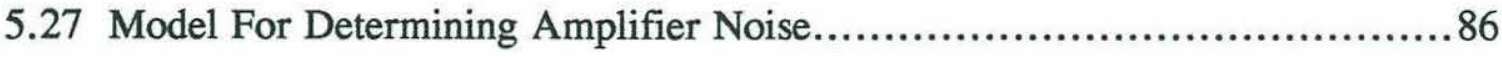

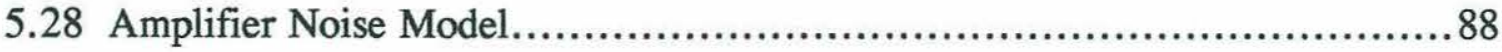




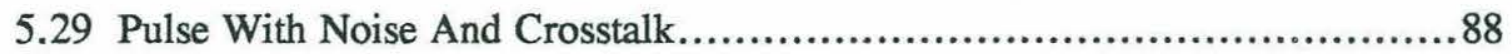

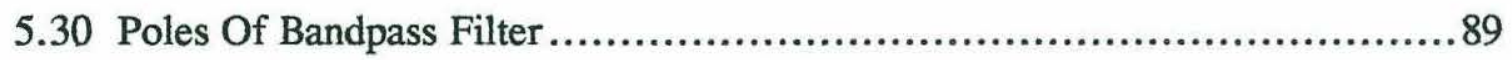

5.31 Bandpass Filter Frequency Response..........................................90

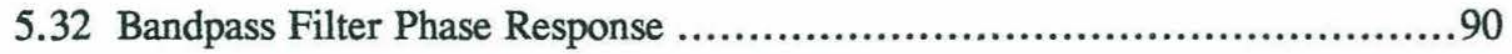

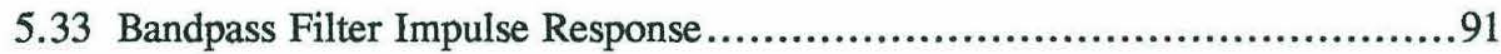

5.34 Received Signal After Bandpass Filter .......................................91

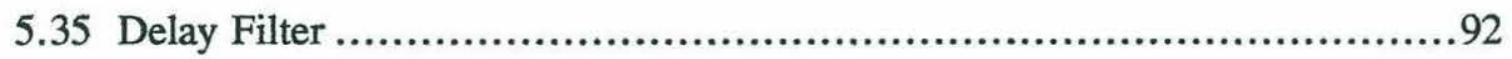

5.36 Compensating Filter Equivalent Circuit .....................................93

5.37 Compensating Filter Frequency Response .....................................95

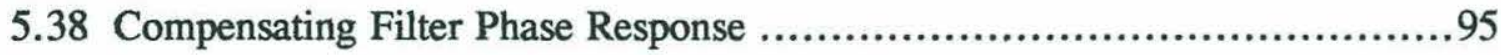

5.39 Compensating Filter Impulse Response ......................................96

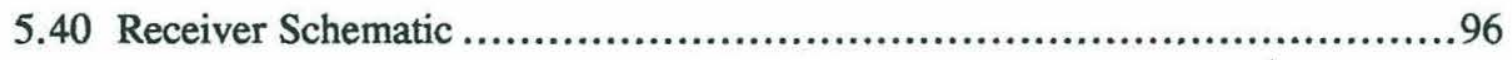

5.41 Final Signal Before Sampling …............................................. 97

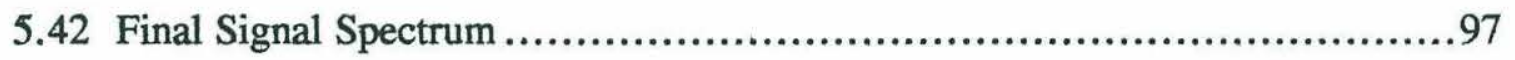

5.43 Final Signal When Transmitted Pulse Is Fed Back.............................98

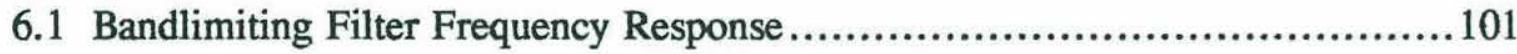

6.2 Inverse Transducer Frequency Response.........................................104

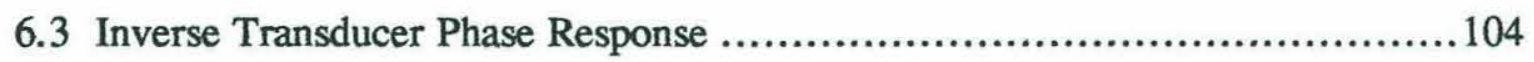

6.4 Forward And Reverse Filtering Of Square Pulse By Inverse Transducer .........105

6.5 Inverse Butterworth Frequency Response .......................................108

6.6 Inverse Butterworth Phase Response............................................ 108

6.7 Forward And Reverse Filtering Of Square Pulse By Inverse Butterworth ........109

6.8 Compensating Filter Inverse Function..........................................112 


\section{Tables}

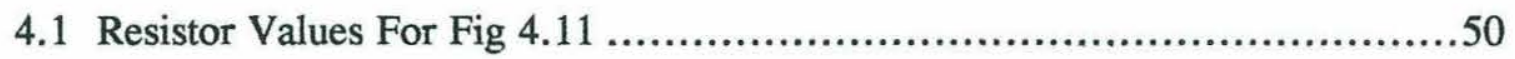

5.1 Component Values For Model Transducer ................................67

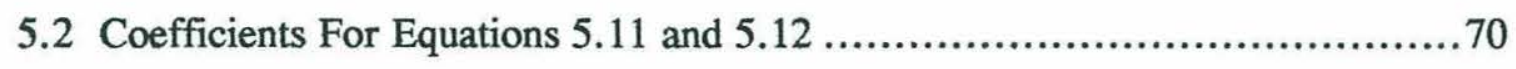

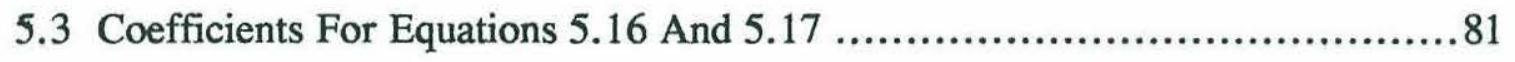

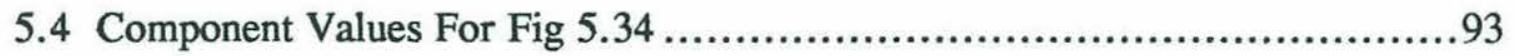

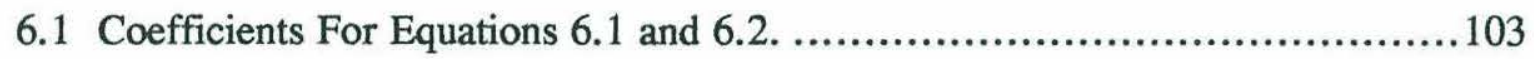

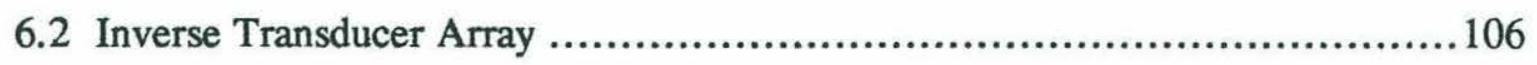

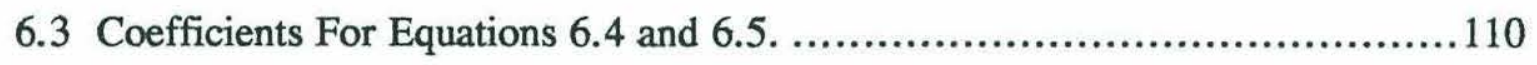

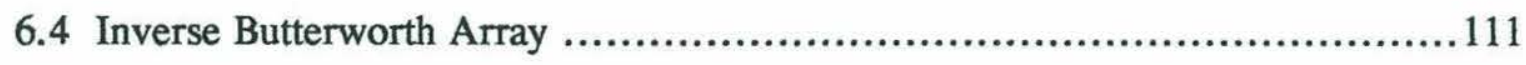

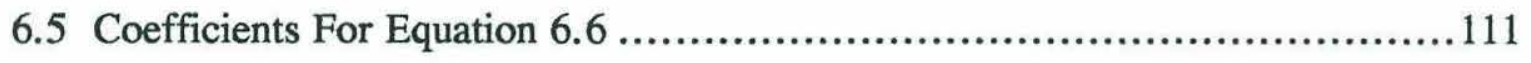




\section{Chapter 1}

\section{Introduction}

\subsection{Motivation}

Exploration of the seafloor has been one of mankind's most important endeavors ever since ocean navigation became important to the survival and prosperity of people and nations. Accurate knowledge of underwater topography has long been important for the safe and accurate navigation of ships. In more recent times, the advent of the submarine as a weapon of war has resulted in the need for even more detailed and accurate survey information. Modern science and archaeology also benefit greatly from the ability to conduct underwater searches and produce high-quality sonar images.

The Woods Hole Oceanographic Institution (WHOI) maintains a fleet of underwater vehicles capable of underwater surveying and imaging. The most famous of these is undoubtedly the remotely controlled JASON vehicle, which has been involved in such well publicized endeavors as the discovery of the World War II battleship Bismark. A variety of towed vehicles exists, used for more routine tasks such as harbor-bottom surveys. These vehicles use a variety of survey instruments, such as underwater cameras and lasers, but among the more versatile sensors is sonar.

The Deep Submergence Laboratory (DSL) has recently developed a family of calibrated high frequency bathymetric sonar systems. This includes a $120-\mathrm{kHz}$ and a $200-\mathrm{kHz}$ system designed for high-resolution sidescan, and a $300-\mathrm{kHz}$ forward 
scanning unit. These systems are similar in design and provide quantitative and well as qualitative data. To extract as much information as possible from this data, it is desirable to have a detailed signal-processing model of the system itself, including the transducers and receiver electronics. This model can be used to remove some of the system-dependent effects from the data. This research is motivated by the need to develop such a model.

\subsection{Research Objectives}

There are two major sections of the system to model: the transmitter and the receiver. The transmitter model attempts to reproduce the process by which the outgoing signal is formed and generates a mathematical description of the pulse. The receiver model simulates the step by step process by which the return is amplified and preprocessed for digital conversion.

The objectives of this research are to analyize the construction and operation of the sonar system and develop this information into a mathematical model A further objective is to explore how this forward model can be used to develop an inverse model suitable for application to received data.

\subsection{Outline of Thesis}

Chapter 2 presents a brief history of bathymetric sonar systems and places the systems under discussion here in their proper historical context. Chapter 3 discusses sonar systems in general and indicates how they can be modeled. Chapter 4 discusses 
the construction and operation of the WHOI $300-\mathrm{kHz}$ sonar. Chapter 5 translates the information from chapter 4 into a detailed mathematical model. Chapter 6 shows how this model can be inverted to recover the original signal. Chapter 7 summarizes thesis results and recommends further research. 


\section{CHAPTER 2}

\section{Background on Sidescan Sonar}

\subsection{Introduction}

Of the many uses man has found for sonar, one of the most important and fundamental for the military, commercial, and scientific applications has been the surveying and charting of the seabed. This has traditionally been done using an echo sounder mounted on a ship's hull. This method, however, is not very effective since only a narrow strip of terrain in the immediate vicinity of the ship can be surveyed. It is possible to partially overcome this limitation through use of a multiple beam system. Even this requires a large number of parallel passes to survey a large area [1].

One way around this difficulty is to tow a submerged echo sounder at a specified height above the bottom with its beam pointed perpendicular to the direction of motion. Such a system can survey a large area in a single swath and can be made to produce an image analogous to an aerial photograph. The drawback to such a system is that it is ambiguous. As can be seen from Fig 2.1, irregular terrain features can lead to incorrect interpretations because the operator has no direct knowledge of the angle from which a particular return is received. Only the travel time, and hence the range, is known directly [1].

There are a number of ways around this problem. For example, two separate sonar arrays, stationed a distance apart, can have their returns combined to create a 


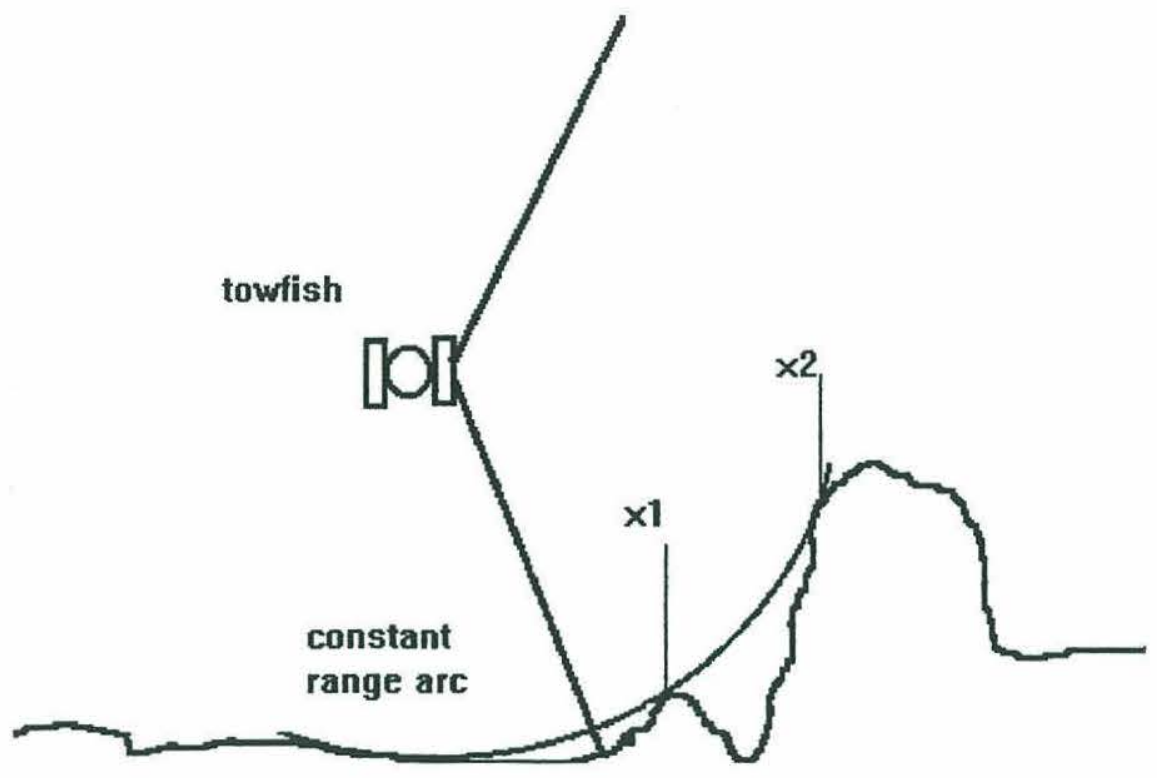

Fig 2.1 With range information only, the actual location from which a return is received is ambiguous when the terrain is irregular. Either $\mathrm{x} 1$ or $\mathrm{x} 2$ could be the actual bottom location.

stereoscopic image. This, however, has not proven very effective due to the large distances required between arrays to achieve sufficient parallax. Moving these arrays through the water while keeping them properly aligned presents tremendous practical difficulties. Even if this could be done, experiments have shown that the correlation in returns between the two arrays tends to be poor due to the complex interference patterns produced by the numerous scatterers present within the ensonified volume [2].

Another possible approach is to combine the data from a ship-mounted multibeam system with the data from a towed sidescan system. This method has been demonstrated by the coordinated operation of the $12-\mathrm{kHz}$ SeaMARC II sidescan sonar and a hull mounted 12-kHz system known as Sea Beam. Although this method has shown promise its overall resolution is limited [1].

The most promising and simplest approach has been to combine the returns from two separate arrays placed a fraction of a wavelength apart. By measuring the phase 
difference between the received signals at the two arrays, the deflection angle from which the return originated can be determined. This method is known as bathymetric sidescan sonar or simply swath bathymetry [3].

\subsection{History}

The idea that depth information could be obtained from the phase differences in received sidescan signals was first developed in the 1960 s by Chesterman et al. [4] and Heaton and Haslett [5]. It was well known that fringe patterns could often be detected in sidescan data due to interference between the direct path return signal and the return signal reflected from the top of the water column. This phenomenon is most apparent when the surface is smooth and is known as the Lloyd mirror effect [5]. The original insight made by these researchers was that each fringe corresponded directly to a particular declination angle and that it is possible to obtain depth information from them. The problem was that such smooth surface conditions seldom exist in practice. The process was further developed by Stubbs et al. [6] by incorporating a reflector above the receiving transducer. Known as "Telesounding", this technique produced a reliable fringe pattern. This was refined by eliminating the reflector and producing the fringe pattern through the use of two closely spaced receivers [3].

The next step was taken by Denbigh [2], who described a system that eliminated the production of interference patterns and measured the phase differences directly. Known by the acronym BASS, for "bathymetric sidescan sonar", this was the first of the "modern" swath-bathymetry systems. Operating at a frequency of $410 \mathrm{kHz}$, BASS used two receive arrays spaced three wavelengths apart along with a separate transmit array. In 1980 a bathymetric system was mounted on a fully instrumented towfish for 
the first time by the Norwegian Continental Shelf Institute (IKU). Known as TOPOSSS , this system operated at $160 \mathrm{kHz}$ and used a two-wavelength transducer separation. At the same time, the University of Bath introduced a $300-\mathrm{kHz}$ system that operated with a 14-wavelength separation for greater resolution (unfortunately, this wider separation results in increased receive-angle ambiguity, as described below). This system produced the first survey results considered reliable enough for inclusion on navigational charts. Members of the University of Bath team later formed a commercial venture known as Bathymetrics Ltd, which produced a product known as the Bathyscan 300. In 1983, an American firm, International Submarine Technology, introduced SeaMARC II, a long range bathymetric sidescan sonar operating at $12 \mathrm{kHz}$. Other commercial units are now available [3].

Work in progress at the Woods Hole Oceanographic Institution's Deep Submergence Laboratory (DSL) aims to build a real-time, three-dimensional model of the undersea environment by combining data from several sensors. These include sidescan and forward-scan sonar, imaging lasers, and stereo photography. Such a model could be used, for example, by a free-swimming autonomous vehicle to navigate. Such an application would require automatic extraction of calibrated, quantitative data. Since previous bathymetric sidescan sonars had provided only qualitative information, new systems are needed to provide calibrated backscatter measurements. Working with Acoustic Marine Systems (AMS) of Redmond, WA and with the University of Washington's Applied Physics Laboratory (APL), DSL has developed and tested several such systems. Among these have been an APL modular $200-\mathrm{kHz}$ sonar designed for use aboard deep-submergence vehicles such as JASON and ARGO. Another is the AMS 120, a stand-alone towed $120-\mathrm{kHz}$ bathymetric sidescan system [7]. 
One of the latest initiatives at DSL has been to combine the operations of a bathymetric sidescan sonar with a forward looking, mechanically scanned bathymetric sonar on a single instrumented, towed sled. This platform is illustrated in Fig 2.2. It employs a new $200-\mathrm{kHz}$ sidescan sonar along with a $300-\mathrm{kHz}$ forward scanning sonar. The forward scan is achieved by mounting the transducer array on a programmable stepper motor. This motor is designed to pause at user specified increments. During this pause, a sonar pulse is transmitted and its return is recorded. The $200-\mathrm{kHz}$ and the $300-\mathrm{kHz}$ systems are similar in design and both were developed at APL. The $300-\mathrm{kHz}$ system contains four identical split-beam arrays, vertically stacked, allowing four horizontally narrow beams to be transmitted simultaneous in different directions, thus reducing the number of step increments required to scan a particular sector (Fig 2.3). This thesis is concerned principally with the design of this $300-\mathrm{kHz}$ system, although most of the results obtained could easily be applied to the $200-\mathrm{kHz}$ system due to their fundamentally similar design.

\subsection{Modeling}

In its broadest sense, modeling is the process of combining available information to build a mathematical description of some system or phenomenon. For underwater mapping, the sources of such information include sonar, laser, photography, or historical knowledge. If more information is added to the description, a more accurate or higher resolution model can be produced.

Stewart [1] describes a methodology, known as "Stochastic Backprojection", for incorporating information with finite uncertainties into an overall model. In developing 


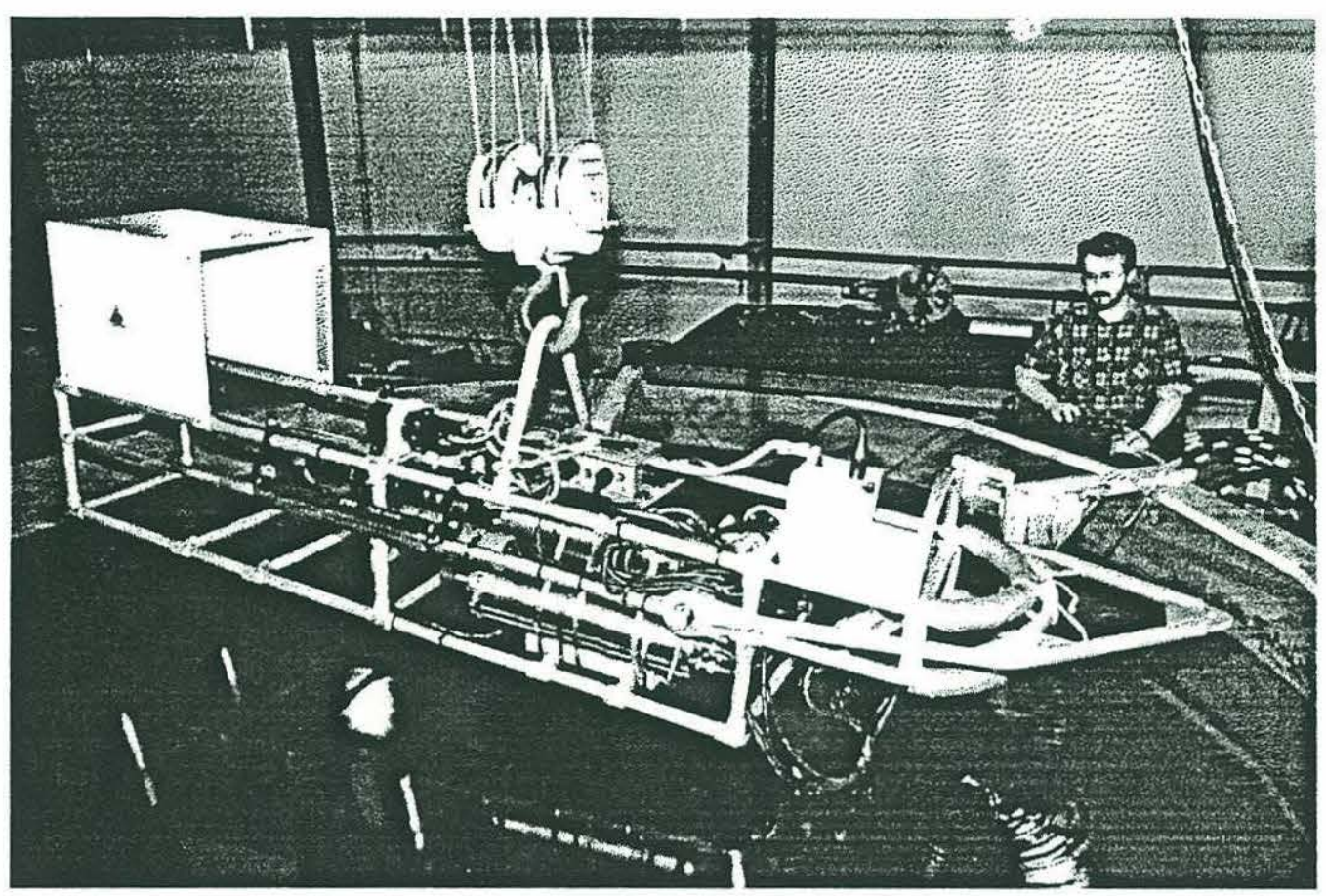

Fig 2.2 The DSL-200/300 towed sonar platform.

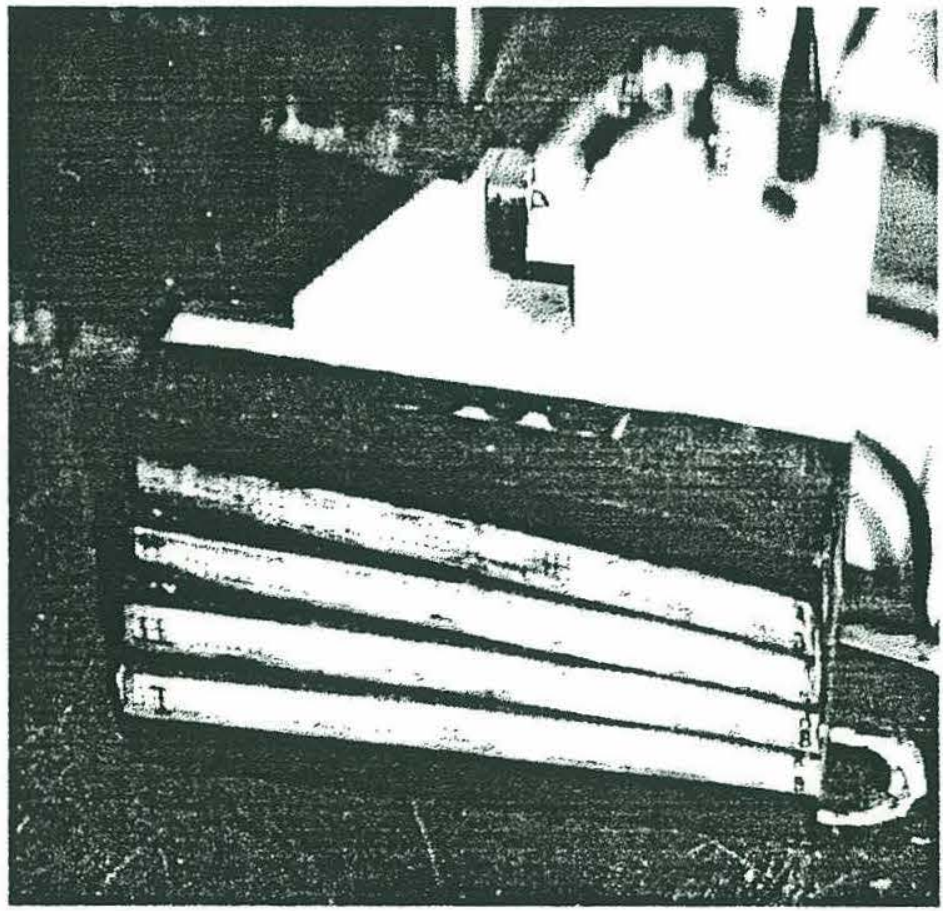

Fig 2.3 The $300 \mathrm{kHz}$ array consists of four vertically stacked bathymetric arrays. 
his model, Stewart develops a particular form of the sonar equation:

$$
\mathrm{RL}=\mathrm{PL}+\mathrm{DI}_{\mathrm{t}}-\mathrm{TL} t+\mathrm{S}-\mathrm{TL} r+\mathrm{DI}_{\mathrm{r}}
$$

where

$$
\begin{array}{ll}
\mathrm{RL}=\text { receiver level } & \mathrm{TL}=\text { transmission losses } \\
\mathrm{PL}=\text { power level } & \mathrm{S}=\text { scattering strength } \\
\mathrm{DI}=\text { directivity index } &
\end{array}
$$

The subscripts $t$ and $s$ represent the transmit and receive characteristics of the system, respectively. Given the assumption of a uniform medium with spherical spreading and absorption losses only, this equation is recast in parametric form:

$$
\mathrm{S}(\tau, \mathrm{xs})=\operatorname{RL}\left(\tau, \mathrm{xr}_{\mathrm{r}}\right)-\left[\mathrm{PL}\left(\tau, \mathrm{x}_{\mathrm{t}}\right)+\operatorname{DIt}\left(\tau, \alpha_{\mathrm{t}}\right)+\operatorname{DI} \mathrm{r}\left(\tau, \alpha_{\mathrm{r}}\right)-\mathrm{TL}(\tau)\right]
$$

Here, alpha is a vector of directional cosines, $\mathrm{x}$ is a position vector, and $\tau$ is the roundtrip travel time for a pulse. The term in brackets, which is a measure of our knowledge of the system and the propagation path, is referred to by Stewart as the "detectivity". The objective is to invert this equation to determine the actual location from which a return is received. Although this gives the impression that the process is completely deterministic, there are also stochastic effects to consider. These include non-zero beam widths, fluctuations in the propagating medium, target scattering variations, and processing differences among various sonar systems.

In order to characterize both the deterministic and stochastic elements of the system, each aspect can be modeled in turn. Much work continues to be done on modeling the physical environment. Such models include sound propagation under various conditions of temperature, pressure, and salinity; characteristics of reflecting 
surfaces; environmental noise; and so on. Often, only simple models of the sonar system itself have been used, representing only such fundamental characteristics such as operating frequency and beamwidth. In this thesis I develop a more comprehensive system model. 


\section{CHAPTER 3}

\section{Generalized Model Of A Sonar System}

\subsection{Introduction}

All sonars, from the simplest depth finder to the most complex military systems, rely on the same basic physical principles and thus have many things in common. The object of this chapter is to describe a "generic" sonar system and to discuss how it can be modeled analytically.

Fig 3.1 shows a simple model of an active sonar system. Some systems will not contain all modules shown here, and some complicated systems will contain many more. In some cases, the functions shown separately here will be combined into one. Beam patterns, for example, are often determined by the physical shape of the transducer elements rather than through separate hardware or software. However, the functions pictured here are important and general enough to be dealt with in detail [8].

\subsection{Transducers}

The most basic element to any system is the transducer. This is simply a device for converting between electrical and acoustic energy. A transmit transducer, often called a projector, converts electrical energy to acoustic energy, while a receive transducer, often called a hydrophone, converts acoustic energy into an electrical signal. A typical application will use a single transducer for both functions. Transducers come in many 


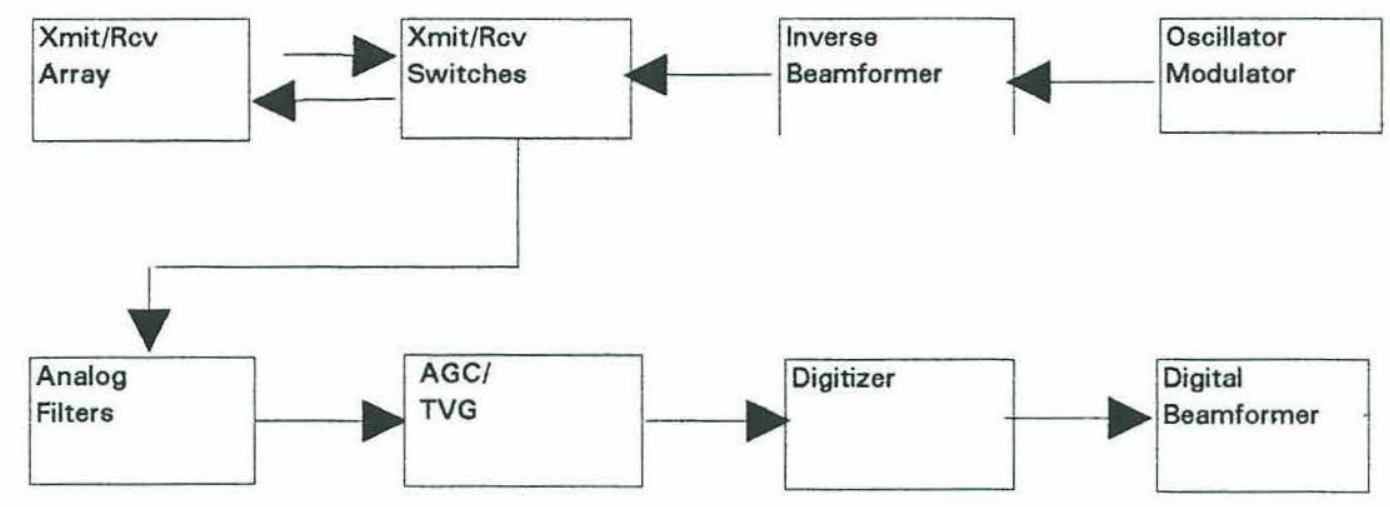

Fig 3.1 A generic active sonar system. Some units, such as beamformers and AGC/TVG (Automatic Gain Control/Time Varying Gain) are not present in all sonar systems.

different sizes and shapes, depending on their application. Low frequency or high directivity, for example, require large transducers. In many cases, two or more transducers will be combined into arrays. Suitable amplitude and phase tapering of these arrays allows specialized beam patterns and spatial focusing.

Three basic types of transducer are in use: (a) magnetostrictive, (b) electrostrictive and (c) piezoelectric. The magnetostrictive type uses a piece of magnetic material that tends to contract along the axis of an applied magnetic field. Thus, application of a magnetic field producing current will establish a corresponding physical vibration in the material, which is then transmitted into the fluid. When the process is allowed to operate in reverse, these devices can also act as receivers. The response of a magnetostrictive material depends only on the magnitude of the applied field and not on the polarity, which results in an overall nonlinear response for the transducer. To obtain a linear response an external polarizing field must be added. An electrostrictive transducer is similar, except that active material responds to changes in the electric rather than the magnetic field. It also suffers the disadvantage of a nonlinear response. Electrostrictive transducers are characterized by a low impedance, which is an 
advantage for an active system where large acoustic power transmission is required. A piezoelectric transducer is similar but has a much higher impedance. It also has a linear response, making it a popular choice in many systems [9].

An important characteristic for any transducer is its bandwidth. This is characterized by the quality or "Q" factor. A high value of $\mathrm{Q}$ indicates a narrow bandwidth, and vice versa. When a short pulse must be transmitted without distortion, a wide bandwidth and thus a low value of $\mathrm{Q}$ is required.

As discussed in Chapter 5, the $300-\mathrm{kHz}$ system being examined here uses piezoelectric transducers. These devices can be modeled as a damped harmonic oscillators (Fig 3.2). A quantity known as the transformation ratio, $\alpha$, can be defined:

$$
\alpha=\frac{\mathrm{Ae}}{1}
$$

Here $\mathbf{A}$ is the surface area of the active element, 1 is its thickness, and $\mathrm{e}$ is the piezoelectric stress coefficient, defined as the stress produced per unit applied electric
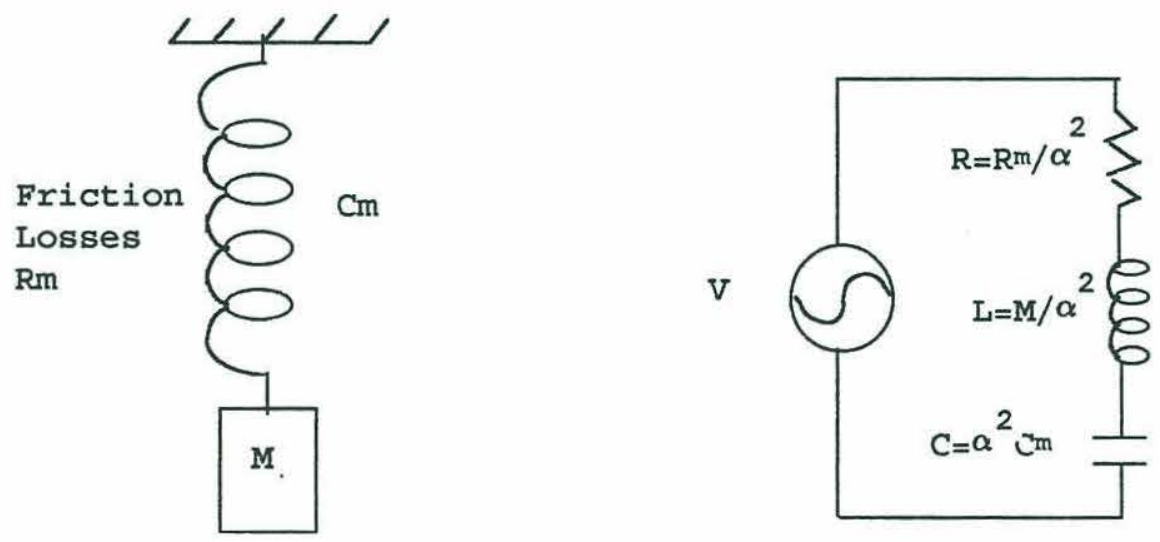

Fig 3.2 A mechanical oscillator and it's electrical analog. This circuit can be used to simulate the response of a transducer. 
field. The transformation ratio relates attributes of the mechanical oscillator to attributes of it's electrical analog. Referring to Fig 3.2, it can be shown that the following relationships hold [9]:

$$
\begin{gathered}
\mathrm{R}=\frac{\mathrm{Rm}}{\alpha^{2}} \\
\mathrm{~L}=\frac{\mathrm{m}}{\alpha^{2}} \\
\mathrm{C}=\alpha^{2} \mathrm{C}_{\mathrm{m}}
\end{gathered}
$$

The above analysis suggests that the series RLC circuit can be used as a basic model for a transducer. The resonant frequency of such a circuit is given by

$$
\omega_{0}=\frac{1}{\sqrt{\mathrm{LC}}}
$$

while the $\mathbf{Q}$ factor is given by:

$$
\mathrm{Q}=\frac{\omega_{0} \mathrm{~L}}{\mathrm{R}}
$$

The resonant frequency should match the operating frequency of the sonar, while $\mathrm{Q}$ can be adjusted to achieve the desired bandwidth. If the band limits are defined by the half-power frequencies with respect to resonance, the bandwidth is given by:

$$
\beta=\frac{\omega_{0}}{Q}
$$




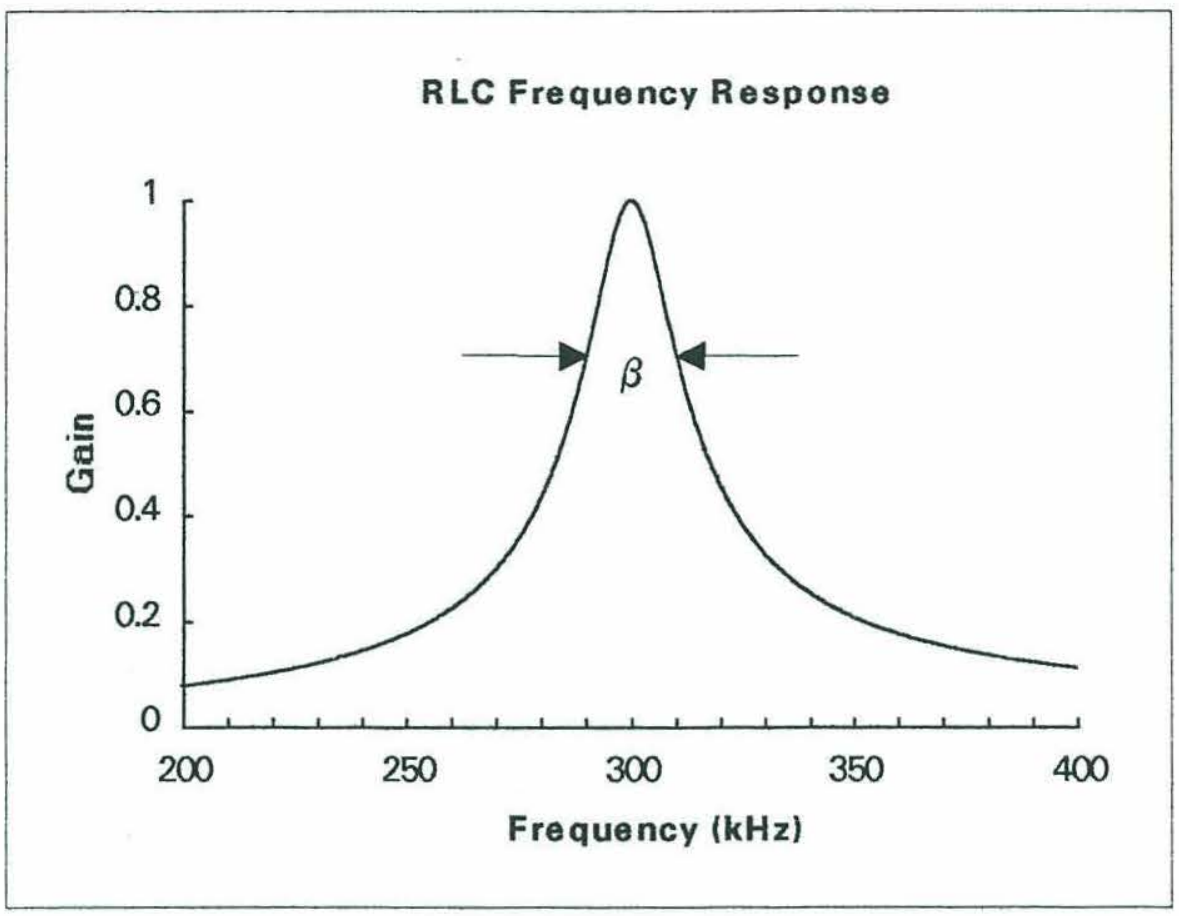

Fig 3.3 Normalized RLC frequency response for a circuit resonant at $300 \mathrm{kHz}$. This response can be used to model a transducer.

The response is illustrated in Fig 3.3 [9].

When constructing the transducer equivalent circuit, account must also be taken of the intrinsic capacitance and resistance of the element. This is illustrated in Fig 3.4, where $C_{o}$ represents the element capacitance and $R_{0}$ represents the element resistance. In practice, the intrinsic capacitance is often tuned out by a shunt inductor [9].

\subsection{Pulse Generation}

Since an active sonar system depends on the transmission of energy into the water, an important part of the generic model is a mechanism for generating a pulse of 


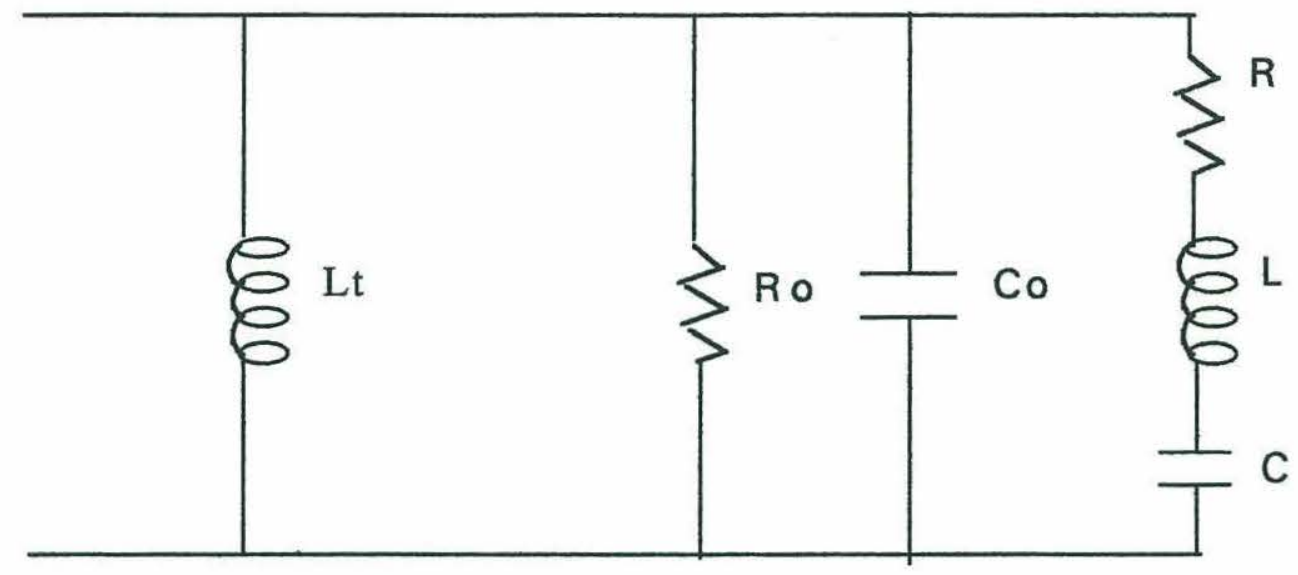

Fig 3.4 Equivalent transducer circuit, with parallel resistance Ro and capacitance Co added. Lt is an optional parallel inductance added by the designer to tune the circuit.

appropriate amplitude, length, shape, and frequency. These parameters depend largely on the sonar's application.

Since a relatively large amplitude means a relatively large amount of energy transmitted into the water, a high amplitude signal is desirable from the point of view of maximizing the energy returned from the target and thus detectability. On the other hand, a large amplitude also tends to increase the reverberation return from the surface, the bottom, and volume scatterers. Furthermore, large amplitudes can also lead to certain undesirable nonlinear effects. Different systems employ a wide range of pulse amplitudes, depending largely on the system at hand. A high-frequency imaging sonar, such as the one discussed in the next chapter, generally requires a large-amplitude pulse to obtain adequate signal-to-noise ratio at long range [8].

A long pulse length is advantagous because it puts a relatively large amount of energy into the water, once again increasing the chance of target detection. Also, a longer pulse requires less amplifier and transducer bandwidth to produce. 
Unfortunately, range resolution is inversely proportional to pulse length. Since high resolution is important for an imaging sonar, its pulse length will be relatively short.

A rectangular pulse is ideal for maximizing target resolution, because it delivers its energy in the shortest possible time. In practice, however, the pulse must be transmitted through amplifiers and transducers with finite bandwidths, so the result will never be ideal.

If two transducers have the same physical dimensions, the one with the higher carrier frequency generally results in narrower beam patterns and thus higher angular resolution. Resolution is further improved because the width of the diffraction pattern produced by the reflecting object is inversely proportional to the sonar wavelength [10]. Unfortunately, higher frequency generally correlates to shorter effective range, due to the oceans tendency to operate as a low-pass filter [11]. Since high resolution is required, the range of the sonars considered here will be limited.

The required signal can easily be generated, for example, with a crystal-controlled oscillator and a suitable high-power, wideband, amplifier. The signal is modulated (with a gating mechanism, for example) to produce the required pulses [8]. Due to the wide bandwidth of modern amplifiers, the pulse delivered to the transducer can be modeled as a nearly square pulse. With an eye toward chapter 5 , and considering the above discussion, a relatively high frequency $(300 \mathrm{kHz})$ will be chosen, along with a short pulse length (300 microseconds). No real amplifier can achieve an instantaneous rise or decay time, but these can be made very short when compared to the pulse length. A large amplitude is required, again anticipating the application in the next chapter. 


\subsection{Beamforming}

Many sonar applications require the transmitted energy to be focused into a narrow beam. This enables greater effective ranges and higher target detectability in the preferred direction. It also reduces the reverberation noise returned from nonpreferred directions. Beamforming techniques are also applied on the receive side, improving resolution and rejecting much of the omnidirectional ambient noise [11].

Consider a continuous and finite line array as depicted in Fig 5.5. If $\mathrm{z}$ represents the spatial coordinate and $\lambda$ is the operating wavelength of the sonar system, a nondimensional spatial parameter $u$ can be defined:

$$
\mathrm{u}=\frac{\mathrm{z}}{\lambda}
$$

A nondimensional angular parameter $\mathrm{s}$ can be defined by reference to the angle $\varphi$ between the normal to the array and the direction of interest:

$$
\mathrm{s}=\sin (\varphi)
$$

A function $\mathrm{B}(\mathrm{s})$, known as the beam pattern, is defined. This function represents the relative amplitude of the pulse in the direction represented by s. It is usually normalized so that $\mathrm{B}^{2}=1$ on the maximum response axis. The function $\mathrm{T}(\mathrm{u})$, known as the taper function, represents an amplitude and/or phase variation imposed on the array elements. This function is normalized so that its integral over the length of the 


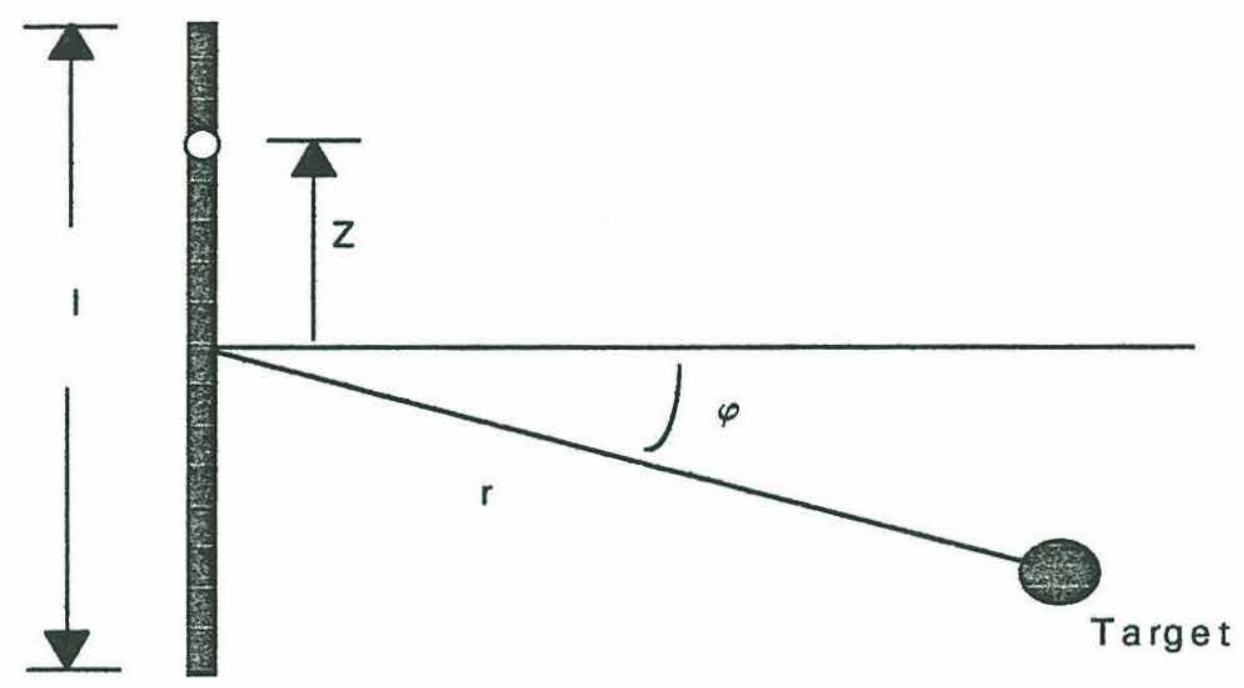

Fig 3.5 The finite linear array. $\mathrm{Z}$ represents the distance from the array center to an infinitesimally small element.

array is unity. It can be shown that the beam pattern and taper function are related as Fourier transform pairs:

$$
\begin{aligned}
& B(s)=\int_{-\infty}^{\infty} T(u) \exp (i 2 \pi s u) d u \\
& T(u)=\int_{-\infty}^{\infty} B(s) \exp (-i 2 \pi s u) d s
\end{aligned}
$$

By properly tapering the amplitude along the array, it is possible to achieve suitable compromises between beamwidth and sidelobe strength. It is also possible to show that by suitably tapering the phase the maximum response axis of the beam can be steered in azimuth angle, $\phi$. Thus, it is possible to electronically scan a sonar beam by varying the phase taper with time. In practice, most arrays are not continuous but are made up of a number of discrete elements [11]. 
A well known characteristic of Fourier transforms is that a relatively compressed pattern in one domain corresponds to a relatively wide pattern in the other domain. When designing an imaging sonar it is desirable to achieve a narrow beam pattern in the plane of the platform but broad in the plane normal to the platform [12]. Therefore, a rectangular pattern is suggested, with the long axis oriented in the plane of the platform and the narrow axis oriented perpendicular to it. This is what will be used for the generic model. The phase and taper will be kept uniform in both directions along the array. The Fourier transform of such a square taper function is known as a "sinc" function, given by

$$
\operatorname{sinc}(x)=\frac{\sin (x)}{x}
$$

This function is illustrated in Fig 3.6 [13].

The simplest method for establishing the desired beam pattern is by building an array of the appropriate shape and using in-line attenuators to achieve the required shading. A more flexible method is to build a generically shaped array (such as a square) which employs selectable elements and attenuators, or use digital post processing of the data to simulate the desired pattern.

\subsection{Receivers}

The signal received by a transducer or hydrophone is typically very small (on the order of microvolts). A receiver is a collection of amplifiers, filters, and demodulators designed to boost this very small signal and condition it for display or digitization. Frequently, several user-selectable gain stages are provided for flexibility. The filters 


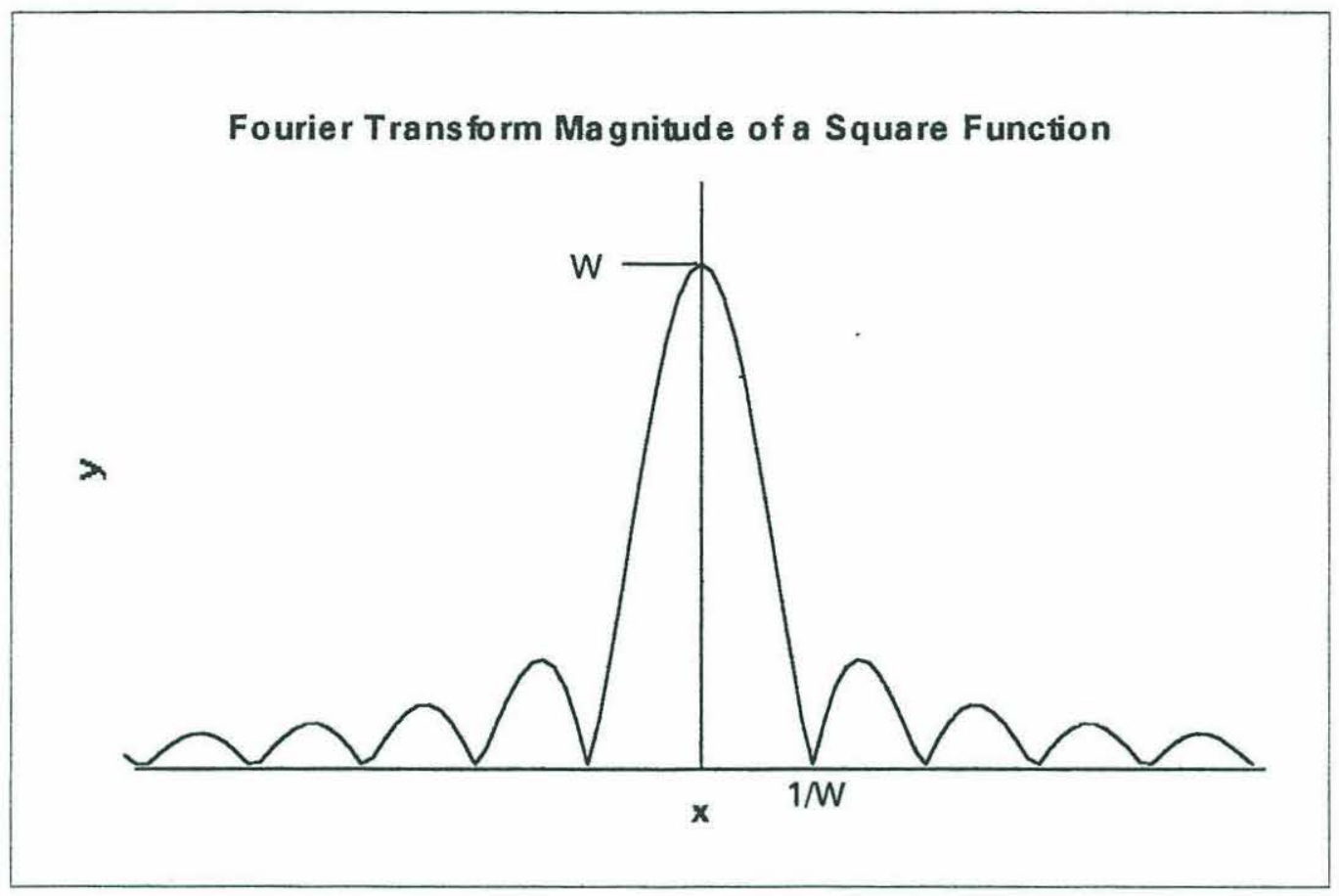

Fig 3.6 Fourier transform magnitude of a rectangular signal with unity amplitude and width $\mathrm{W}$. This function can be used to represent the beam pattern of a uniform line array.

remove ambient noise and other unwanted signals which lie outside the frequency band of interest. A band-limited signal is also desirable for systems that require digital data transmission [8].

The band-limiting filter is placed close to the transducer in the system, allowing out of band noise to be suppressed before amplification in subsequent stages [11]. While a narrow bandwidth is desirable from the standpoint of eliminating unwanted noise, the bandwidth must be wide enough to accommodate signals of the length and rise time expected. It must also accommodate Doppler shifting of the signal caused by relative motion between the target and the sonar. The relationship between the frequency emitted by a stationary target and the frequency measured by a moving observer is given by: 


$$
\mathrm{fr}_{\mathrm{r}}=\mathrm{f} \frac{(\mathrm{c}+\mathrm{vs})}{\mathrm{c}}
$$

where $f_{r}$ is the received frequency, $f_{t}$ is the frequency emitted by the target, $c$ is the speed of sound in the medium, and vs is the radial velocity of the source relative to the target [10]. Assuming a speed of sound in the water of $1500 \mathrm{~m} / \mathrm{s}$, this amounts to no more than a $6-\mathrm{kHz}$ bandwidth requirement for a $300-\mathrm{kHz}$ system, even for velocities as high as $15 \mathrm{~m} / \mathrm{s}$. Therefore the actual bandwidth is more likely to be determined by the need to accurately reproduce the pulse shape.

While an ideal filter would have an instantaneous rise time and a perfectly flat response in the passband, no physical filter can achieve these conditions. Fortunately, there are many analog filters available that come acceptably close. Butterworth, Chebyshev, and Elliptic type filters are the most common examples. For the generic model, the receiver will be represented by a three-pole-pair butterworth filter. Its bandwidth will be equal to ten percent of the center frequency, as illustrated in Fig 3.7. Many receivers will include an additional element called time-varying gain (TVG). This feature provides an increase in the receiver gain, starting from the beginning of the "listen" cycle, which is reset with each pulse. The purpose is to compensate for the attenuation caused by spherical spreading and absorption in seawater. In some cases, TVG will be added during post processing analysis [8].

\subsection{Post Processing}

It is worth noting that several other steps are usually required to render sonar information usable. For example, the data must somehow be presented to an operator 


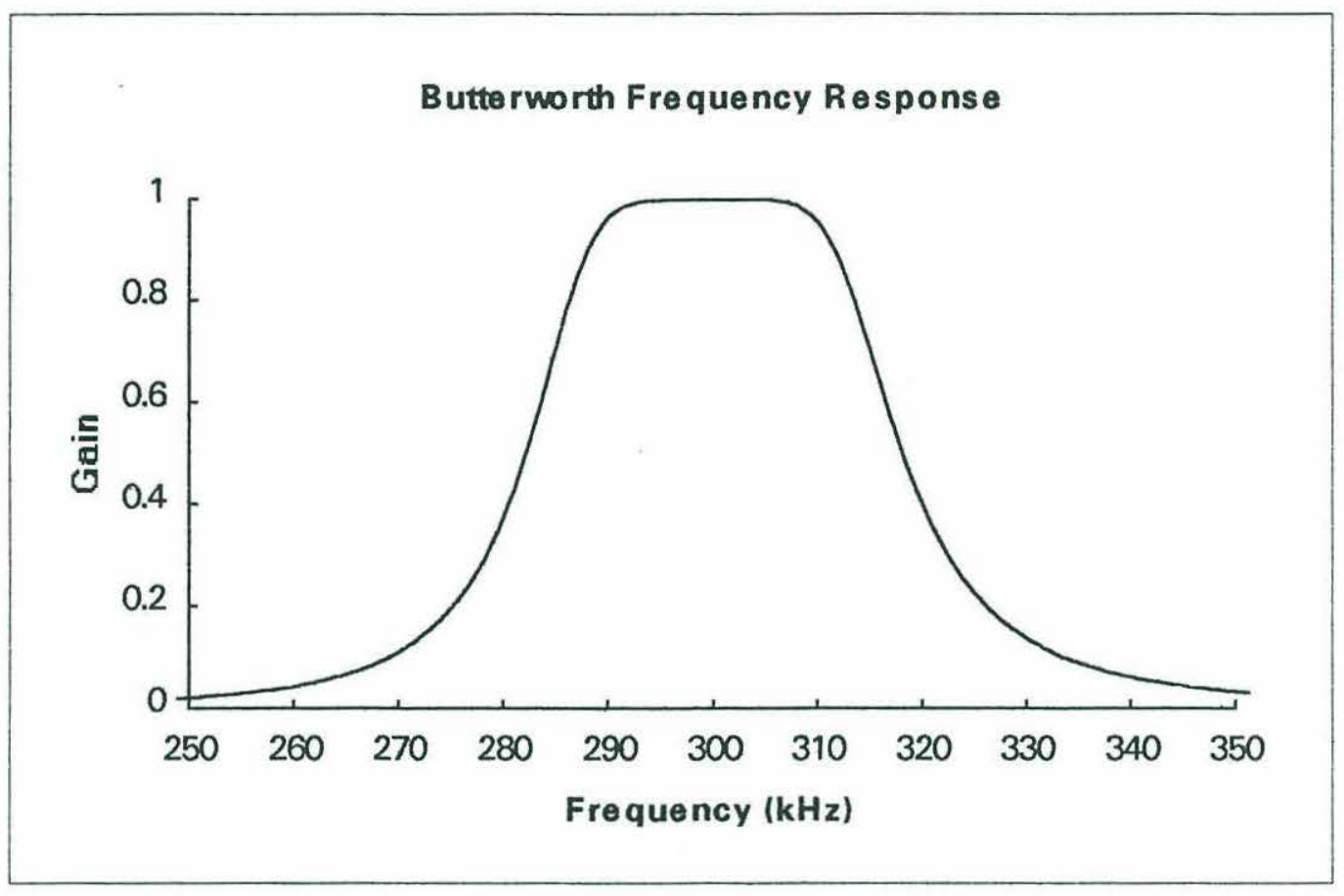

Fig 3.7 Frequency response of a three-pole-pair butterworth filter.

or analyst in a useful form. For some systems, this can be as simple as an oscilloscope trace of the receiver output. Towed bathymetric systems require a more sophisticated display.

Since the sonar is usually towed at great depth, some means of data transmission to the surface is required. Reliable transmission over such great lengths of cable requires that the data be digitized by some suitable sampling technique. In some systems, this digital data stream is modulated onto an analog carrier by frequency-shift keying (FSK), which is sent to the surface on a standard coaxial cable. Newer systems send the digital data directly over fiber-optic cables.

Lastly, bathymetric sonar data can often be made more useful through advanced digital processing and enhancement of the images. This can include the integration of 
data from several sensors, combining several different look angles from the same sensor, or using the inverse system model developed in Chapter 6 to remove system dependent effects. 


\section{CHAPTER 4}

\section{THE DSL BATHYMETRIC SONAR SYSTEM}

\subsection{Introduction}

As mentioned in the previous chapter, the Deep Submergence Laboratory (DSL) at the Woods Hole Oceanographic Institute (WHOI) has worked with the University of Washington's Applied Physics Laboratory (APL) to develop a calibrated bathymetric system that integrates both forward and sidescan data. This concept is illustrated in Fig 4.1. This chapter will explore in detail the construction and operation of this system.

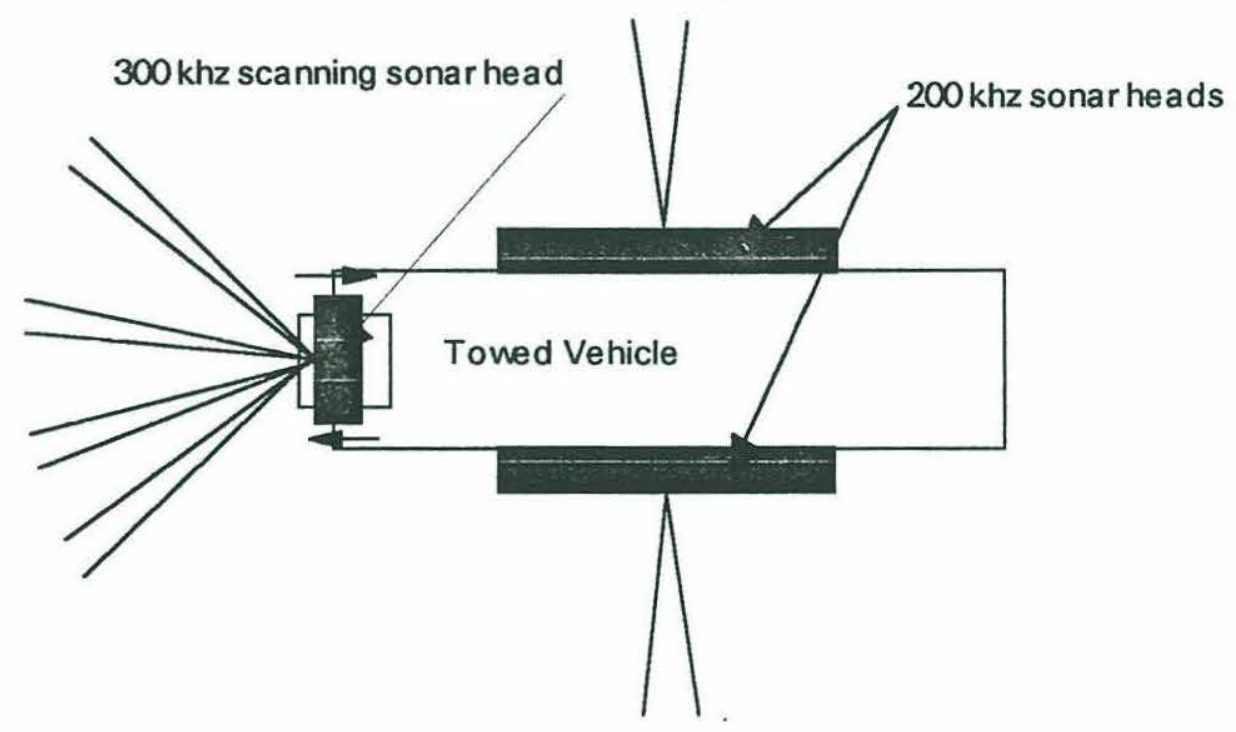

Fig 4.1 The DSL calibrated bathymetric sonar system integrates forward scan and sidescan data. 


\subsection{The DSL-200/300 Sonar Sled}

The DSL 200/300-kHz sonars are supported by the DSL sonar sled, as illustrated in Fig 4.2. The system is controlled by its central processing unit (CPU), a 68HC11E microcontroller. The CPU is supported by software stored in both an internal EEPROM and an external EPROM. It interfaces through four universal asynchronous receiver transmitters (UARTs) to the forward-scan sonar, the sidescan sonar, a magnetic compass, a pressure (depth) transducer, an attitude suite, and the forward scanning motor controller. The software supports two modes of operation: a run cycle and an interactive mode. The run cycle, which is the normal mode, is based on a timing signal from the forward scanning motor controller. When the CPU receives this signal, it pings the sonars, awaits a return, receives data from the auxiliary sensors, and sends information to the serial uplink. The interactive mode allows user control of individual components and is mostly useful for troubleshooting.

The $200-\mathrm{kHz}$ sidescan sonar provides a single one-degree-wide beam. The array actually consists of two vertically stacked transducers. The top transducer transmits and both receive, forming the phase-difference configuration discussed in Chapter 2. The $300-\mathrm{kHz}$ forward-scan unit employs four sets of two vertically stacked transducers, thus providing four one-degree-wide beams, each employing the split-beam concept. The stepper motor is designed and programmed to move in variable increments, positioning the array with at least a .25 degree accuracy at each location. The operator can program the motor to pause for the length of time desired, consistent with the required scan rate and forward range. Both sonars digitize their data at $10 \mathrm{kHz}$ using a scheme known as "quadrature downsampling", which will be discussed in some detail later. 


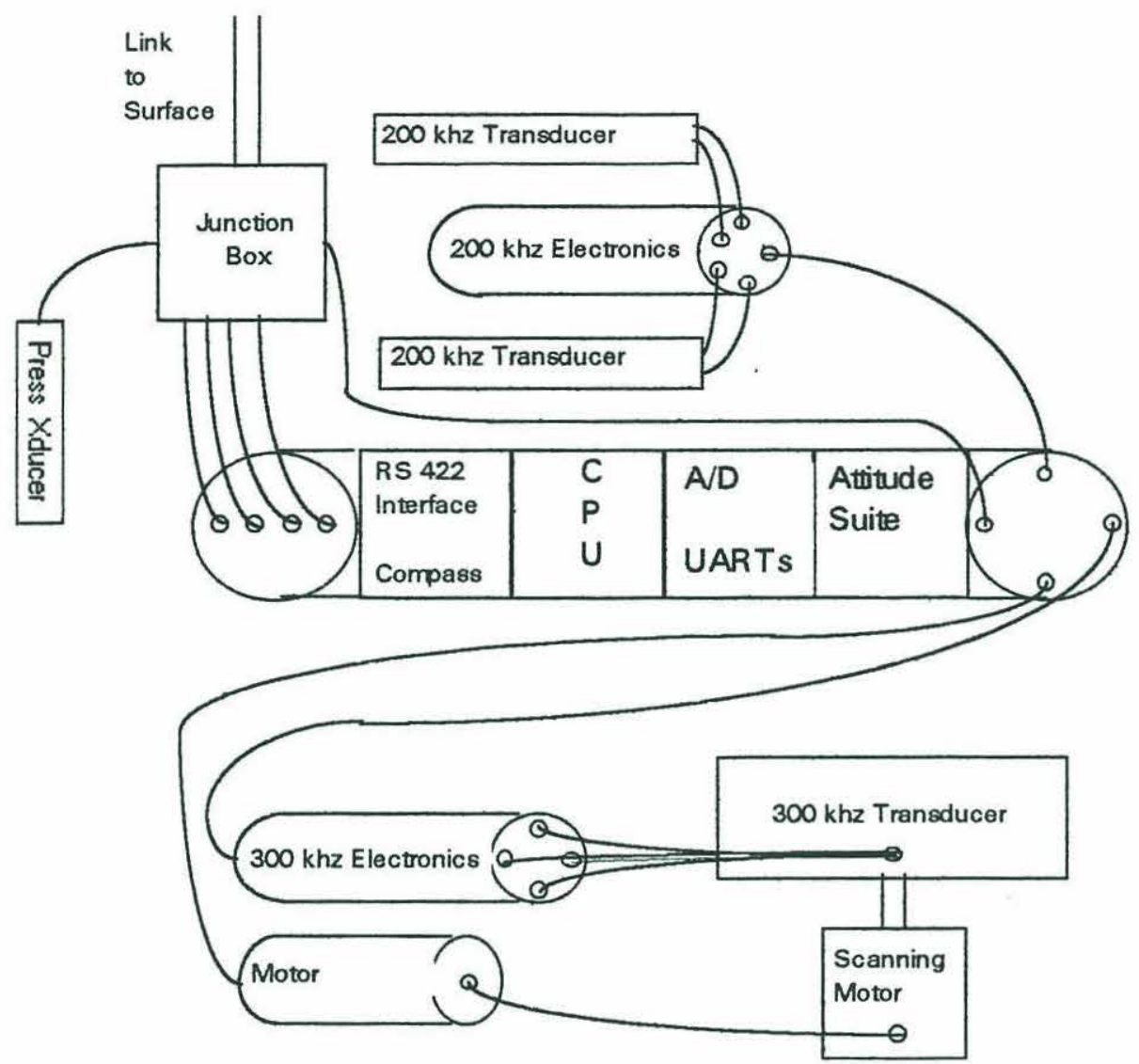

Fig 4.2 The DSL-200/300 towed sonar platform.

The combined digital data from the $200 / 300 \mathrm{kHz}$ sonars and the auxiliary sensors are transmitted by an RS-422 interface to the surface via fiber-optic cables at 9600 baud. Here, the surface processor extracts and displays the auxiliary data and converts the sonar returns into a suitable form for real-time graphics display. The operator can also transmit instructions for the CPU back down the link. The surface processor is programmed to modify the received signal as required. It may be desirable to add time varying gain (TVG), for example, or to compensate for the effects of the system using the results of the model developed by this thesis. 


\subsection{DSL 200/300-kHz Sonar Operation}

In the last chapter, I described the operation of a generic sonar system. I now consider in detail the construction and operation of the DSL-200/300 kHz systems. The discussion refers to the system block diagram given in Fig 4.3.

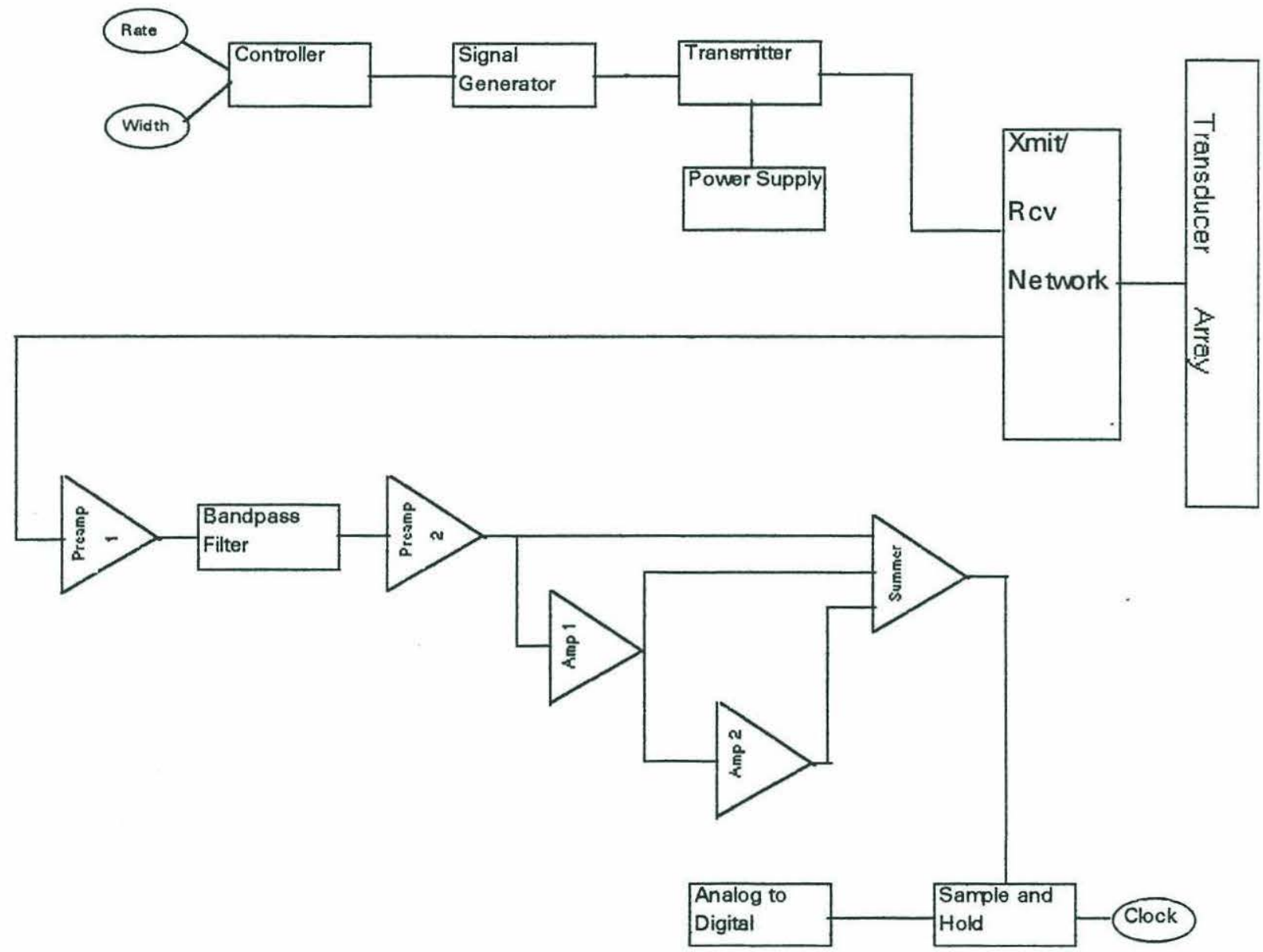

Fig 4.3 Block diagram of the DSL-200/300 khz sonar systems

The microprocessor-based controller provides the interface between operator and sonar. The operator can control the transmitted pulse by varying the ping rate and the pulse width. The pulse width can be set to $100,200,300$, or 600 microseconds to 
achieve the optimum tradeoff between range and resolution. The transmitted power is fixed, although the transmitted energy varies with pulse length. The controller also regulates the signal path through the receive circuitry.

The controller applies 5-V logic levels to the signal generator, where the transmitted pulse originates. Here, customized waveforms are stored in memory chips from which they are clocked out at $12 \mathrm{mHz}$. A series of frequency dividers reduces this to its final value of 200 or $300 \mathrm{kHz}$. The waveforms consist of two square, $5-\mathrm{V}$ logic pulses. The leading edge of the second pulse follows the trailing edge of the first pulse by $100 \mathrm{~ns}$. The two pulses are clocked out separately on two parallel data lines, as illustrated in Fig 3.4.

The parallel pulses are delivered to the transmitter, which is essentially a class B power amplifier (Fig 3.4) [14]. The heart of this circuit consists of four field-effect transistors (FETs) organized in two pairs. The first pair is responsible for producing the positive half of the output waveform, while the second pair produces the negative half. The first part of the waveform produced by the signal generator controls the gate voltages on the first pair of FETs through a solid-state driver interface. The FETs are driven into full conduction when the pulse is high (5 volts) and are fully turned off when the pulse is low ( 0 volts). The FET source is grounded, while the drain is connected to the primary side of an impedance-matching transformer. The other end of the primary coil connects to an 80 -volt, in the case of the $300-\mathrm{kHz}$ sonar, or a 75 -volt, for the $200-\mathrm{kHz}$ sonar, regulated power supply. Several large electrolytic capacitors, both in the power supply and transmitter circuits, supply backup current capacity. The second part of the signal is produced in like manner by the second part of the logic pulse operating with the second bank of FETs. In this case, however, the drain current 


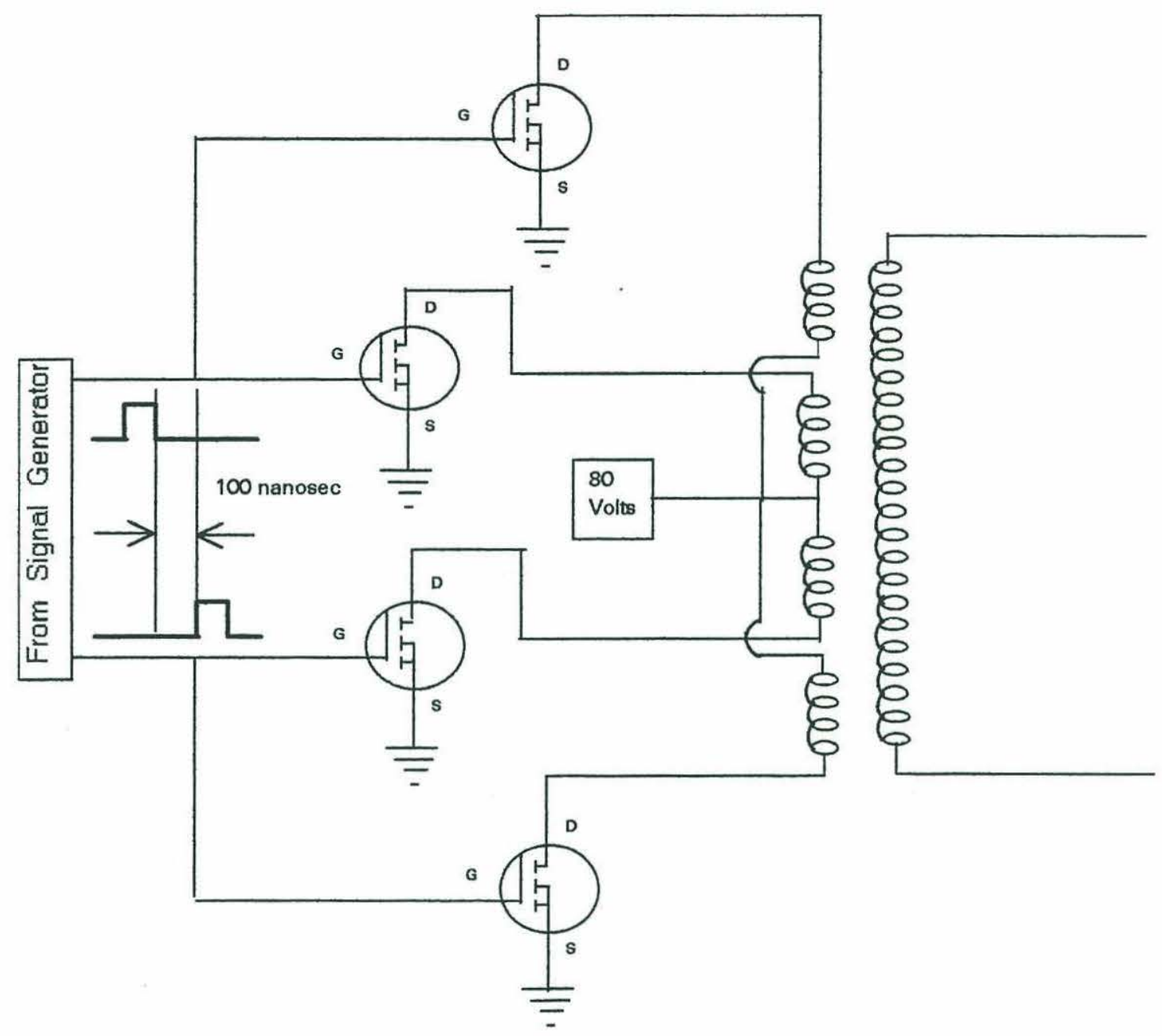

Fig 4.4 The transmitter is a class B type amplifier. The top pair of FETs conduct the positive half cycle, while the bottom pair conduct the negative half cycle. Each half cycle is controlled by a five-volt logic signal with a 100 nanosecond separation between the two.

is directed in the opposite direction through the transformer windings, thus producing the negative part of the waveform.

It should be apparent that the signal delivered to the transformer primary is a largeamplitude (approximately 80 volts) square wave. However, due to the finite bandwidth of the transformer and the transducer, the high-frequency components of the squarewave are eliminated, resulting in an approximately sinusoidal waveform in the water. 
The transmit-receive network serves to protect the sensitive receiver circuitry from the high-powered transmit pulse (Fig 3.5). This network is basically a system of diodes connected to provide an effectively open circuit to the large amplitude transmitter pulse. The small signal (on the order of microvolts) received by the transducer, however, cannot reach the nominal 0.6 volts needed to bring the diodes into conduction, and are thus blocked. During the transmit cycle, the receiver is effectively shorted out of the circuit. During the receive cycle, the signal must travel from the transducer to the receiver across the $1600 \mathrm{pF}$ capacitor, the reactance of which generates the voltage input to the preamp.

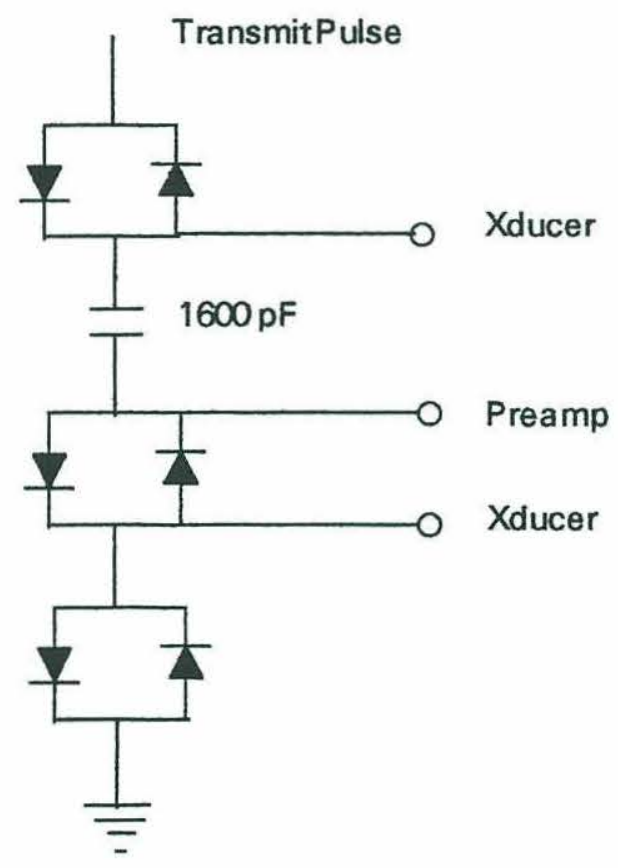

Fig 4.5 Transmit/Receive network. The diode pairs will pass the high-voltage transmit signal while blocking the small-amplitude received signal. The capacitor will affect both the transmitted and received signals, as illustrated in Chapter 5.

The transducer converts the electrical energy of the transmitted pulse into acoustic energy. It also generates electrical energy in response to the signal returned from the 
target. The piezoelectric transducers employed in the $300 \mathrm{kHz}$ system were designed and manufactured at the University of Washington. The active material is lead zirconate-titanate, an alloy known as PZT-4. The transducer is of the half-wave, airbacked type. This can be conceptualized as two quarter-wavelength-thick elements mounted back to back, with the front element in contact with the water and the rear element in contact with air. Since the acoustic impedance of air is very high, most of the back-radiated energy is reflected. Since the reflection process introduces a 180degree phase shift, the wave arrives back at the quarter-wave interface in phase with the forward radiated signal and interferes constructively. This produces an effective doubling of forward transmitted sound pressure level (SPL) [9].

The measured $\mathrm{Q}$ of the transducer is a combination of its mechanical and electrical responses [9]. The electrical $Q$ is determined not only by the resistance and parallel capacitance of the transducer but also by the inductances and capacitances present in the matching transformers and transmit-receive network. Because of this, it is possible for the overall frequency response of the transducer to differ between the transmit and receive cycles. This turns out to be the case, as illustrated by the graphs in Figs 4.6 and 4.7. These plots, which illustrate the bandpass characteristic of the device, were generated at the University of Washington's sonar calibration facility. This facility is mounted on the self-propelled barge R/V Henderson, moored on Lake Union in Seattle, Washington. It has the capability to measure beam pattern amplitude with a $+/-0.5$ $\mathrm{dB}$ accuracy, with an angular resolution of $+/-0.05$ degrees [15]. The bandwidth, $\beta$, of the transducers can be determined by measuring between the $3 \mathrm{~dB}$ down points on either side of the resonant frequency, wo. Using these parameters, $Q$ can be determined by: 


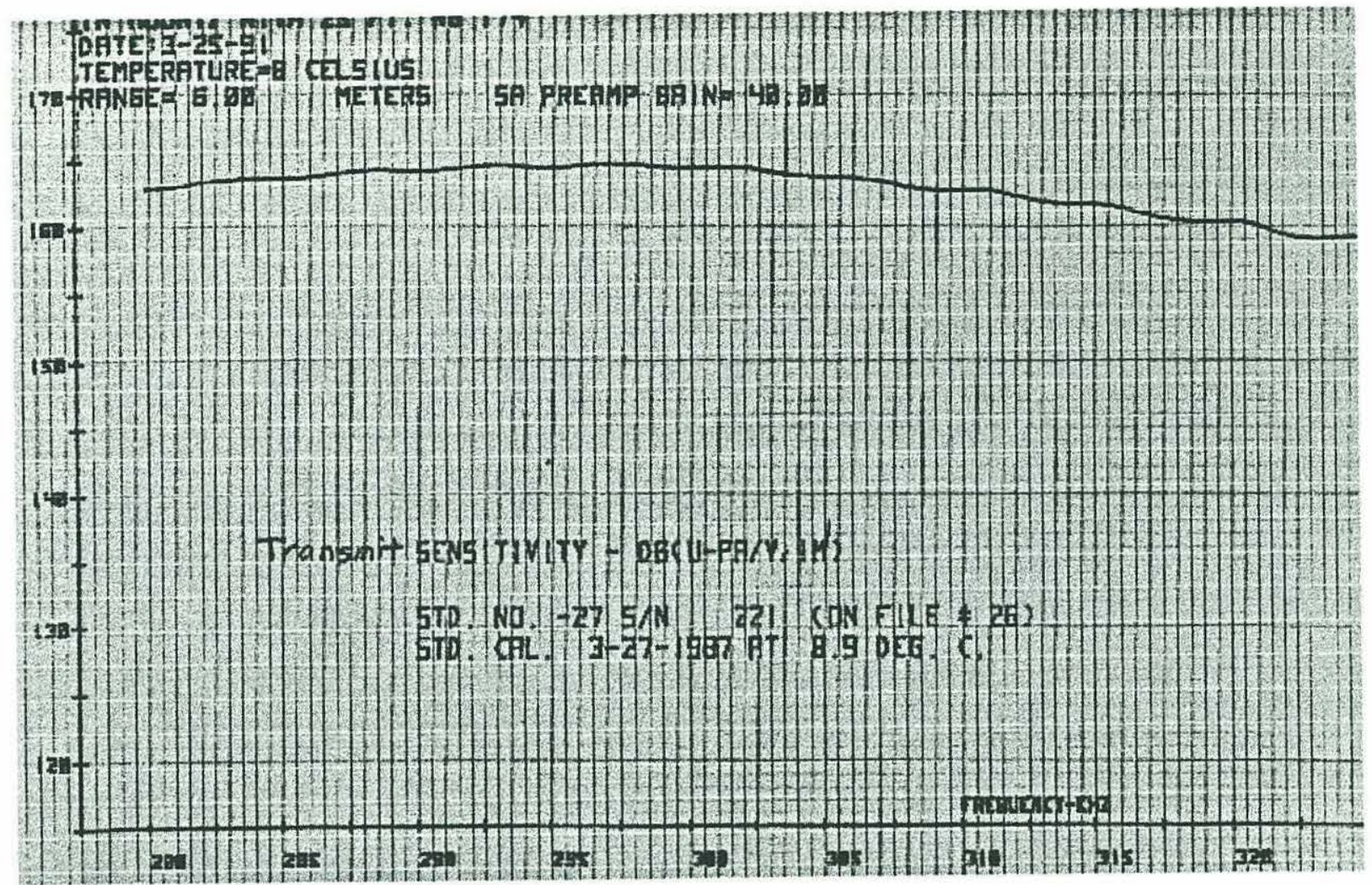

Fig 4.6 Transmit response of the $300-\mathrm{kHz}$ transducers as determined at the University of Washington's Applied Physics Laboratory.

$$
\mathrm{Q}_{0}=\frac{\omega_{0}}{\beta}
$$

Applied to the curves shown, this equation results in a $\mathrm{Q}$ of 8.8 , centered at 297.5 $\mathrm{kHz}$, for the transmit cycle. For the receive cycle, the $\mathrm{Q}$ is 7.7 , centered at 314.0 $\mathrm{kHz}$. These low values of $\mathrm{Q}$ indicate a broadband response, which is desirable to maximize the pulse reproduction fidelity of the system.

The same facility was used to measure the source level (Ls) of the array. From this, the transmitted power can be determined from: 


$$
\mathrm{Lw}=\mathrm{Ls}-171
$$

Using the measured Ls of $201 \mathrm{~dB}$ re $1 \mathrm{~m} \mu \mathrm{Pa}$, this results in an Lw of $30 \mathrm{~dB}$ re $1 \mathrm{~W}$. This can be converted to a power $\mathrm{p}$ in watts by using:

$$
\mathrm{p}=\mathrm{w}_{\mathrm{ref}} \exp (\mathrm{Lw} / 10)
$$

This indicates a transmitted power of $1000 \mathrm{~W}$. A crude measure of the transducer's efficiency can be obtained by comparing the power delivered to the device with the radiated power:

$$
\eta=\frac{\mathrm{v}^{2}}{\mathrm{rp}}
$$

where $r$ is the real resistance of the transducer, as measured at APL, $v$ is the voltage delivered by the transmitter, and $\eta$ is the efficiency. With $\mathrm{v}=680 \mathrm{~V}$ (determined from the transformer turns ratio), $\mathrm{r}=161.3 \Omega$, and $\mathrm{p}=1000$ watts, the efficiency is $34.9 \%$ $\lceil 11\rceil$.

The transducer elements are rectangular, $0.5 \mathrm{~cm}$ by $6.5 \mathrm{~cm}$, and are each composed of 16 smaller sections. Four elements mounted end to end form a long, narrow array. As discussed in the previous chapter, this results in a beam pattern that is broad in a plane normal to the array and narrow in the plane of the array. Fig 4.8 and Fig 4.9 show the beam patterns at $300 \mathrm{kHz}$ as determined at the APL test tank. Measuring at the 3-dB down points, the beam widths of the main lobes are determined as 1.4 degrees in the narrow direction and 61 degrees in the wide direction. The narrow pattern has side lobes at least $11 \mathrm{~dB}$ down. 


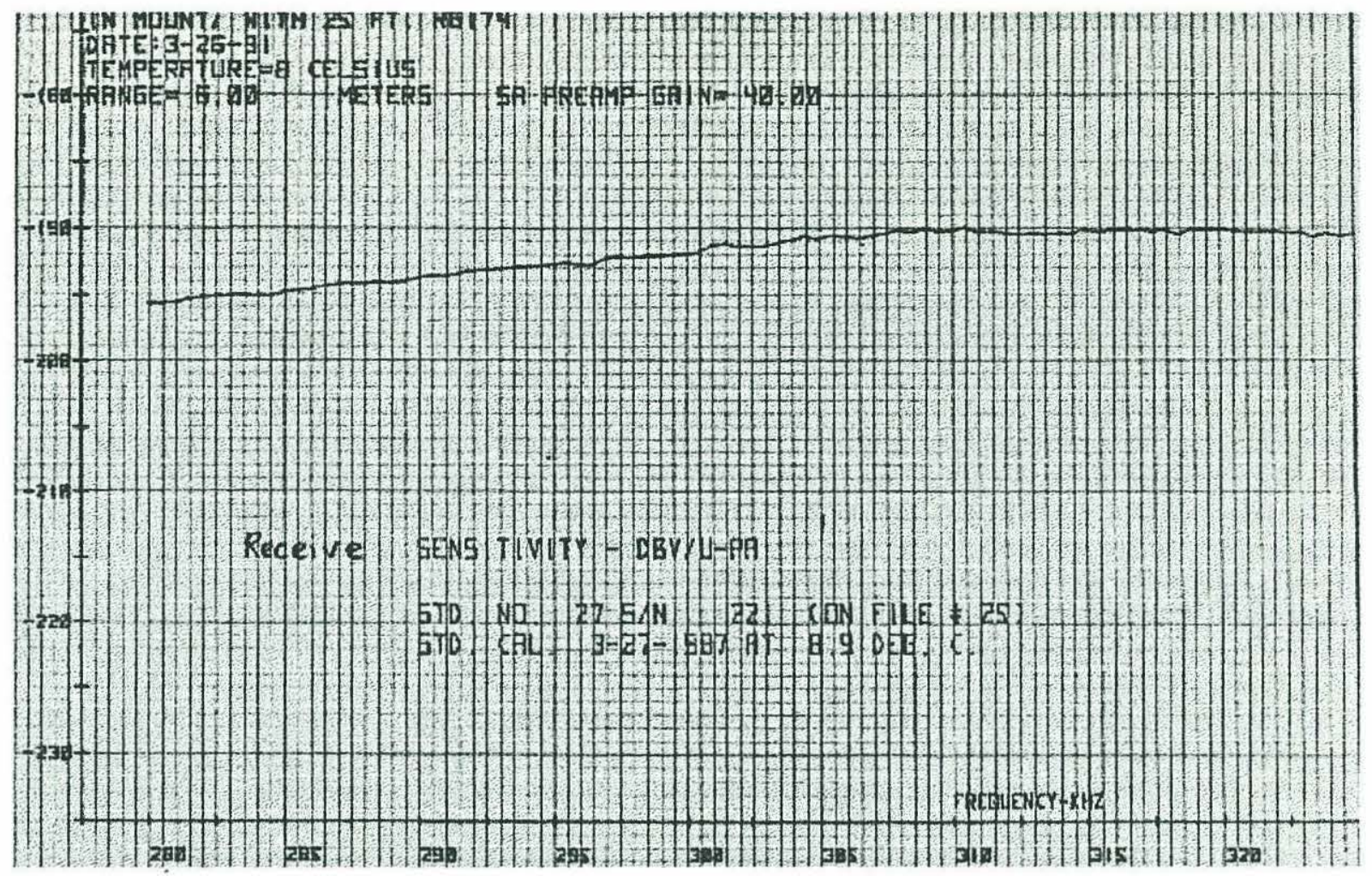

Fig 4.7 The receiving response of the $300-\mathrm{kHz}$ transducers as determined at APL.

The arrays are organized in vertical pairs with a spacing of 1.1 wavelengths between the centers of the top and bottom rows. The phase difference between the two halves can be used to determine the angle from which a return is received. Since the phase difference is only determined modulo $2 \pi$, there is a limited sector over which the return bearing can be unambiguously determined. This sector can be determined from:

$$
\varphi=\mathrm{kd} \sin \alpha
$$

Here $\varphi$ is the phase difference, $\mathrm{k}$ is the wavenumber, $\mathrm{d}$ is the transducer separation, and $\alpha$ is the bearing angle. Substituting the numbers given above, this results in a sector of 
$+/-26.7$ degrees. The actual elevation angle from which a return is received must be inferred from an assumed range annulus, as illustrated in Fig 4.10 [16].

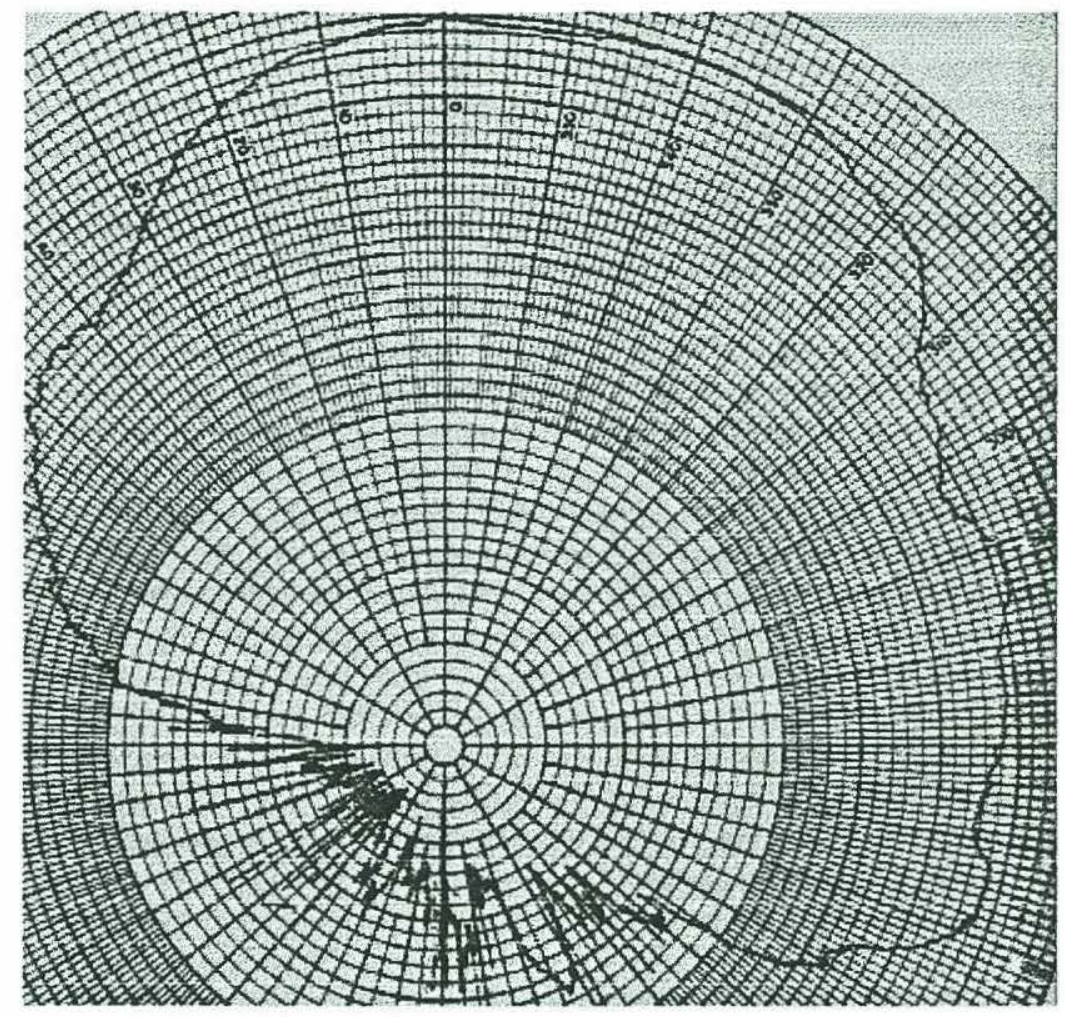

Fig 4.8 Transducer array beam pattern in the vertical direction, as measured at the APL test facility. The scale here is $1 \mathrm{~dB} /$ division, with the peak at $-160.9 \mathrm{~dB}$.

The receiver circuitry, illustrated in Fig 4.11, consists of a series of operational amplifiers, some of which can be switched in or out of the signal path by the controller to achieve the desired gain. The first two amplification stages are enclosed in a metal case to protect these especially noise-sensitive stages from spurious signals and crosstalk from adjacent boards. Each amplifier stage is constructed around Linear Technology's LT1028 ultra-low-noise, high speed, operational amplifier. 


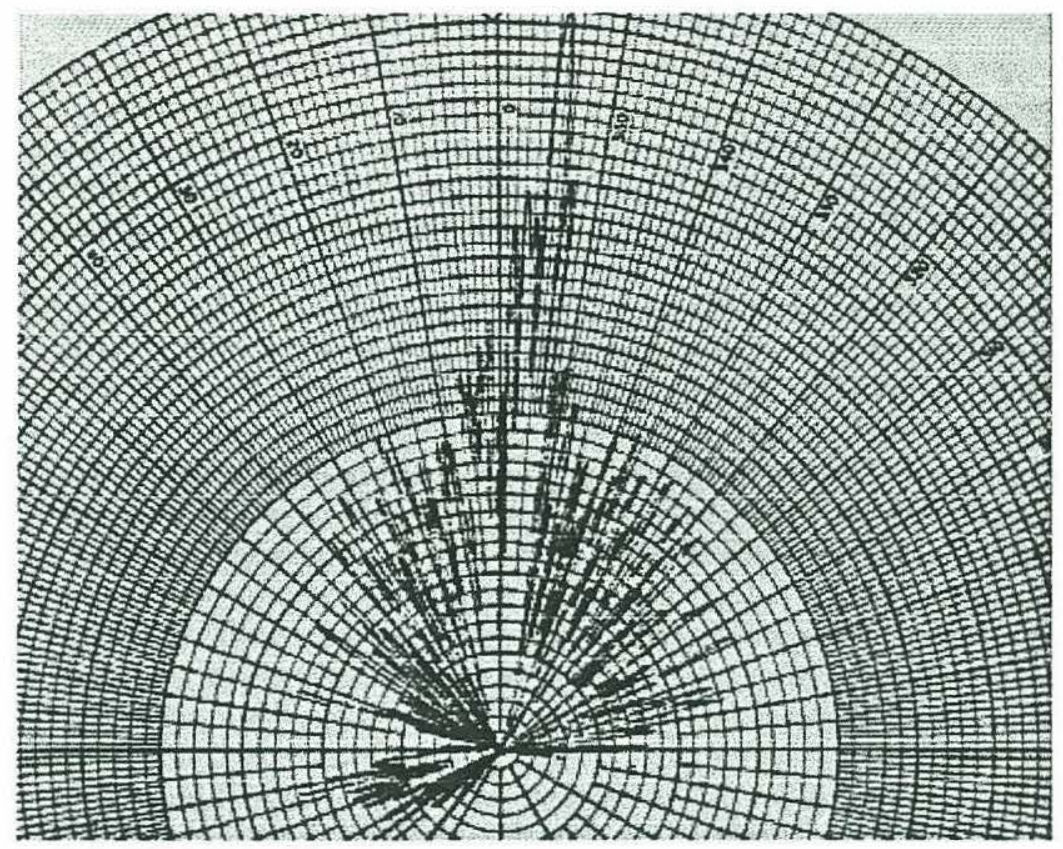

Fig 4.9 Transducer beam pattern in horizontal direction. The scale is $1 \mathrm{~dB} /$ division, with the peak at 167.3.

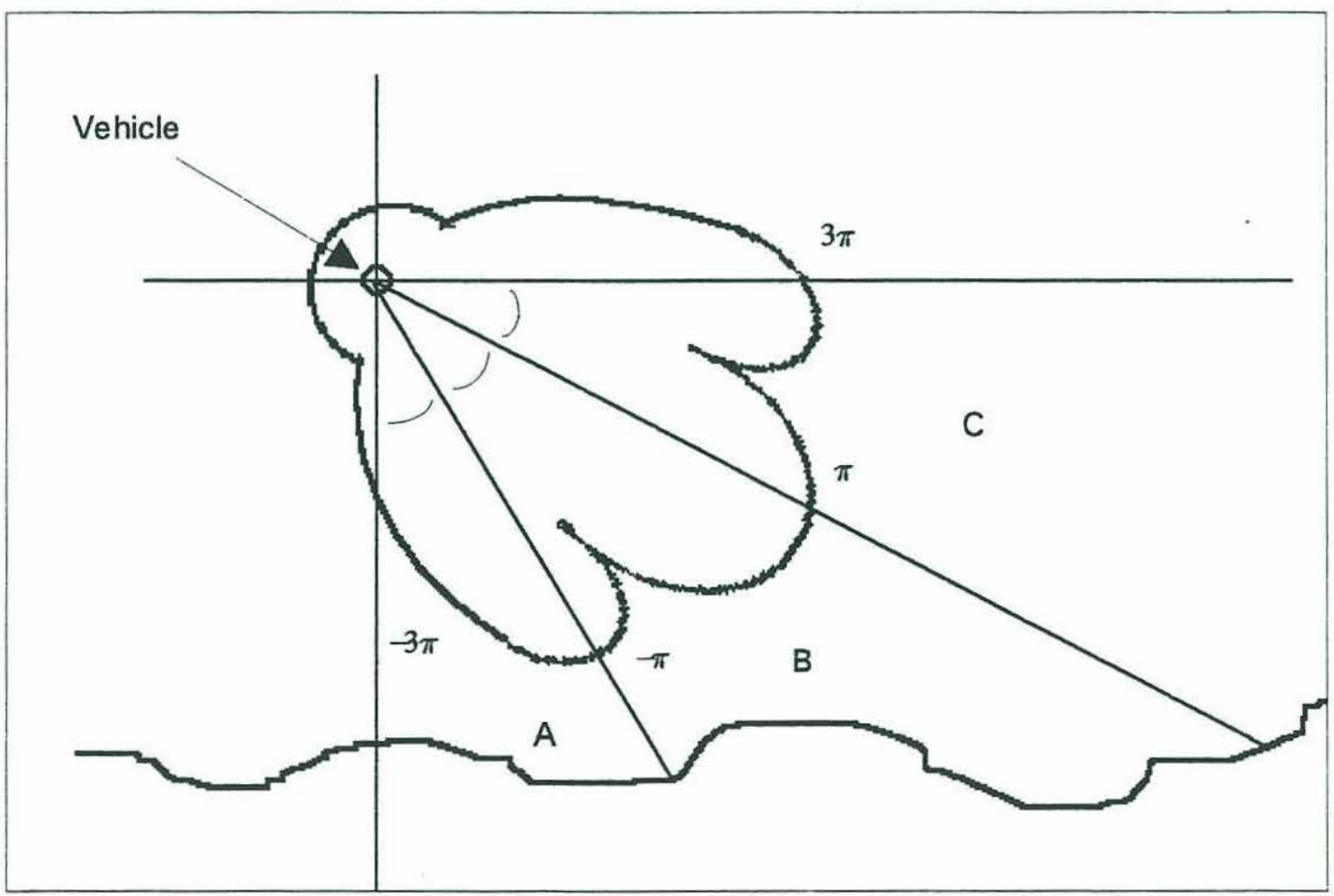

Fig 4.10 The actual return angle may fall into several ambiguous sectors. The actual elevation angle can only be inferred from the range. 


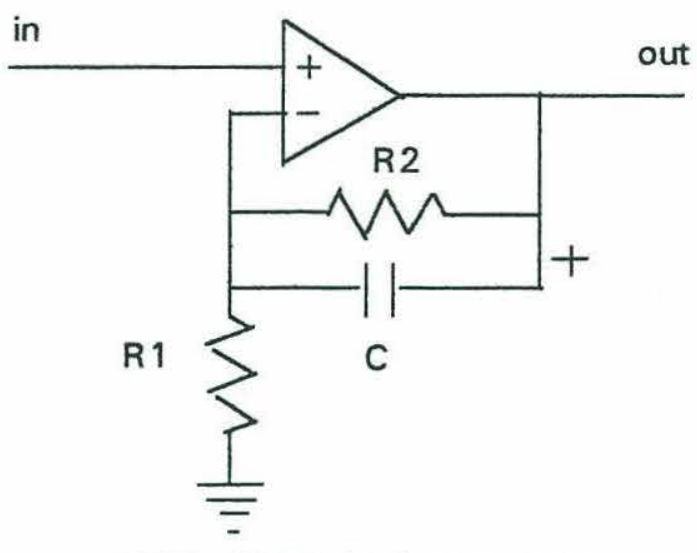

NON - INVERTING AMPLIFIER

A

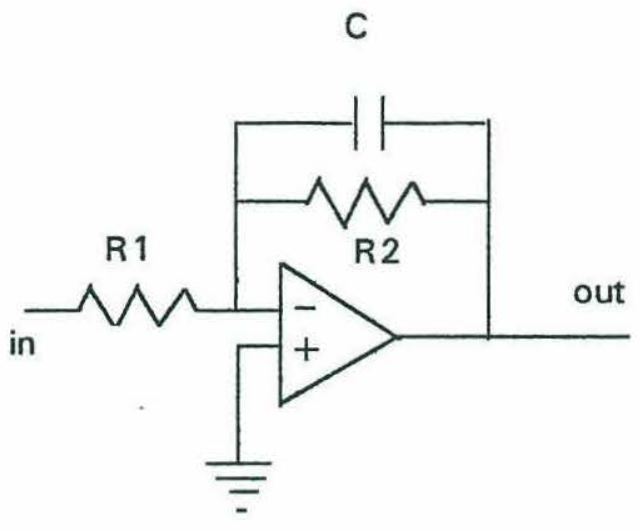

INVERTING AMPLIFIER

B

Fig 4.11 Noninverting (a) and inverting (b) amplifier configurations. The capacitor serves to stabilize the circuit at high frequencies. Resistor values for each stage are given in table 4.1.

\begin{tabular}{|lll|}
\hline & $\underline{\text { R1 }}$ & $\underline{\mathbf{R 2}}$ \\
Preamp 1 & $0.499 \mathrm{~K}$ & $4.53 \mathrm{~K}$ \\
Preamp2 & $2.0 \mathrm{~K}$ & $20.0 \mathrm{~K}$ \\
Amp 1 & $0.499 \mathrm{~K}$ & $4.02 \mathrm{~K}$ \\
Amp 2 & $0.499 \mathrm{~K}$ & $4.53 \mathrm{~K}$ \\
Sum 1 & $2.0 \mathrm{~K}$ & $4.02 \mathrm{~K}$ \\
Sum 2 & $2.0 \mathrm{~K}$ & $4.02 \mathrm{~K}$ \\
Sum 3 & $4.02 \mathrm{~K}$ & $4.02 \mathrm{~K}$ \\
\hline
\end{tabular}

Table 4.1 Resistor values for Fig 4.11 for each amplifier stage. The summing amplifier behaves as an inverting amplifier for each of it's three branches. 
The signal received from the transducer is fed back through the T/R switching network and coupled to the first-stage preamplifier via an isolation transformer. The first stage op-amp is configured as a noninverting amplifier. Referring to Fig 4.11a, the gain of such an amplifier is given by:

$$
\mathrm{G}_{\mu}=\frac{\mathrm{R} 1}{\mathrm{R} 2}+1
$$

Substituting the values shown in Fig 4.11a yields $a G_{\nu}$ of 10.1 for the first stage. Equation 4.6 applies only to an ideal op-amp, but a real device will also have an input capacitance. This creates a pole at the origin in the complex s-plane, an undesirable characteristic. A parallel capacitor is therefore provided in the feedback loop to cancel this pole [14].

The first stage output is directed to a commercially produced bandpass filter, a three-pole-pair butterworth type centered at $300 \mathrm{kHz}$ (200 kHz for the DSL 200) and $30 \mathrm{kHz}$ wide (20 kHz for the DSL 200). The purpose of the filter is twofold: to provide a band-limited signal for later digitization and to limit ambient noise from outside the frequency range of interest.

Output from the bandpass filter is directed through a solid-state switch to the input of the second-stage preamplifier. The solid-state switch either passes the signal or diverts it to ground as determined by a logic signal from the controller. A 2000-ohm resistor to ground just prior to the second stage, combined with the complex impedance of the filter and a 1000-ohm resistor further upstream, act as a voltage divider with a gain of $1 / 2$. The second-stage op-amp itself is configured as an inverting amplifier (Fig 4.11b). The gain is given by: 


$$
\mathrm{G}_{\mu}=\frac{-\mathrm{R} 1}{\mathrm{R} 2}
$$

Using values from Fig $4.11 \mathrm{~b}$, we find that $\mathrm{G}_{p}=-10$ for this stage. Once again, the parallel-feedback capacitor is required for stability.

The signal leaving the second stage now divides into two possible paths determined by the controller through a series of solid-state switches. The controller determines these switch settings based on the operator selected gain. The operator has three choices: $0 \mathrm{~dB}, 20 \mathrm{~dB}$, and $40 \mathrm{~dB}$. If $0 \mathrm{~dB}$ is selected, the signal passes through to the output without further amplification. The total receiver gain in this case is the total gain of the first two stages and voltage divider: 50.5.

If the $20-\mathrm{dB}$ setting is selected, the signal is allowed to proceed in parallel through both the original $0-\mathrm{dB}$ path and through a second path containing a noninverting amplifier stage (Amplifier 1). Using equation 4.6, the gain of this stage is equal to 9.06. The signal path again divides following the amplifier, with the primary path proceeding through to the output.

When $40 \mathrm{~dB}$ is selected, the secondary path from the $20-\mathrm{dB}$ amplifier is activated. Here another noninverting amplifier is provided (Amplifier 2). This stage has a gain of 10.08 .

The three signal paths described above are combined by a final summing amplifier. The output of this circuit is simply the inverted sum of the inputs. Recalling that the 
signal has already been inverted once (in the second stage-preamplifier), the output from the receiver will be approximately in phase with the input from the transducer.

Since the separate signal paths must pass through different numbers of solid-state devices, there will be a slightly different delay associated with each path. Since it is desirable for the signals to be in phase when they are combined by the summer, a simple RC lowpass compensating network is provided in the $0-\mathrm{dB}$ and $20-\mathrm{dB}$ paths. The linear phase characteristics of this circuit in the frequency range of interest serve to provide compensating delays to ensure equal propagation times in all paths.

The output from the summing amplifier is now ready for digitization. This is accomplished in two steps. The sample/hold amplifier "captures" the signal at a specific point in time and "holds" it as a charge on a capacitor. The analog to digital converter senses the DC output from the sample/hold amplifier and generates a digital representation of the voltage level for serial transmission to the surface and digital signal processing.

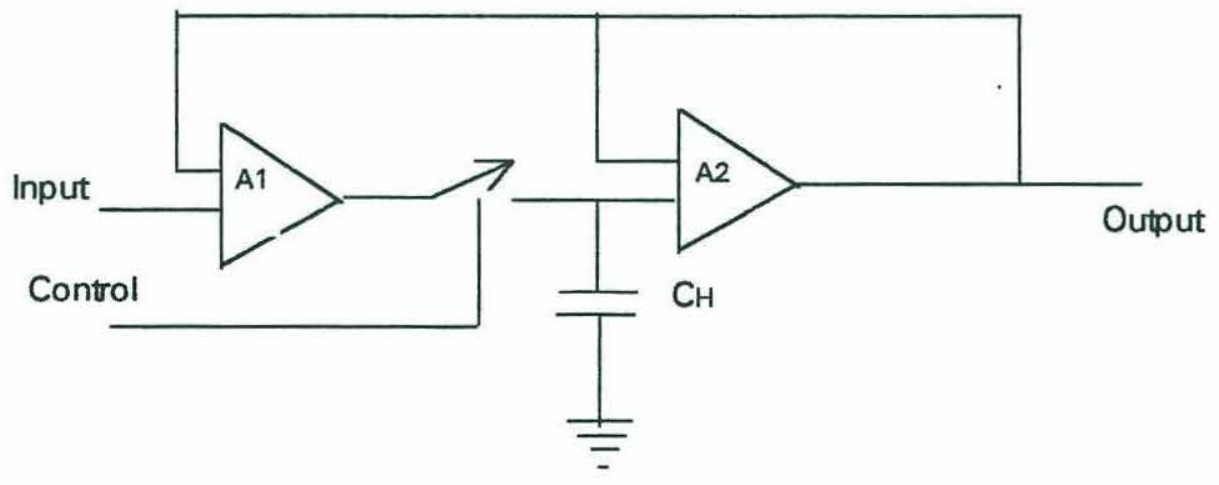

Fig 4.12 An ideal sample-and-hold amplifier.

Fig 4.12 is a schematic representation of an ideal sample/hold amplifier. A1 acts as an input buffer, provides a high-impedance load to the signal source, and supplies 
charging current to capacitor $\mathrm{C}_{\mathrm{H}}$. Switch $\mathrm{S}_{1}$ opens and shuts in response to an external clocking signal (the "mode-control" signal), which determines the sample rate. Amplifier A2 acts as a high-impedance load for the capacitor and as a low-impedance driving source for an external load. A real sample/hold device will have a finite signal acquisition time due to the limited slew rate of the amplifiers and the charging and settling time of the capacitor. The Burr-Brown SHC5320 High Speed Sample/Hold Amplifier utilized in the DSL 200/300 has an acquisition time of 1.5 microseconds [14].

For the digitized signal to be uncorrupted by aliasing, conventional sampling must proceed at the Nyquist rate of twice the maximum frequency present. Since a $660 \mathrm{kHz}$ sampling rate is unnessasarily high for a system bandlimited to $30 \mathrm{kHz}$, an alternate scheme was used [8].

It is possible to take advantage of the bandlimited character of the signal. As mentioned previously, the return is passed through a $30 \mathrm{kHz}$ wide butterworth filter, centered at $300 \mathrm{kHz}$ (refer to the frequency spectrum shown in Fig $4.13 \mathrm{~A}$ for an arbitrary signal $\mathrm{X}(\mathrm{t}))$. Although a real signal is depicted here for convenience, the procedure outlined here could be applied to a complex signal by considering it's real and imaginary parts separately. Sampling at a rate equal to the bandwidth, B, will produce a replica of the complex envelope of the original signal centered at zero. This is illustrated in Fig 4.13B. Since the information content is contained entirely in the complex envelope, the sampling requirement has been reduced by a factor of 10 in this case. Fig 4.13C demonstrates that a further reduction will cause aliasing. Due to symmetry, the information content of the signal is contained in only half the bandwidth. Fig 4.13D shows that if the negative half could somehow be eliminated, 
A

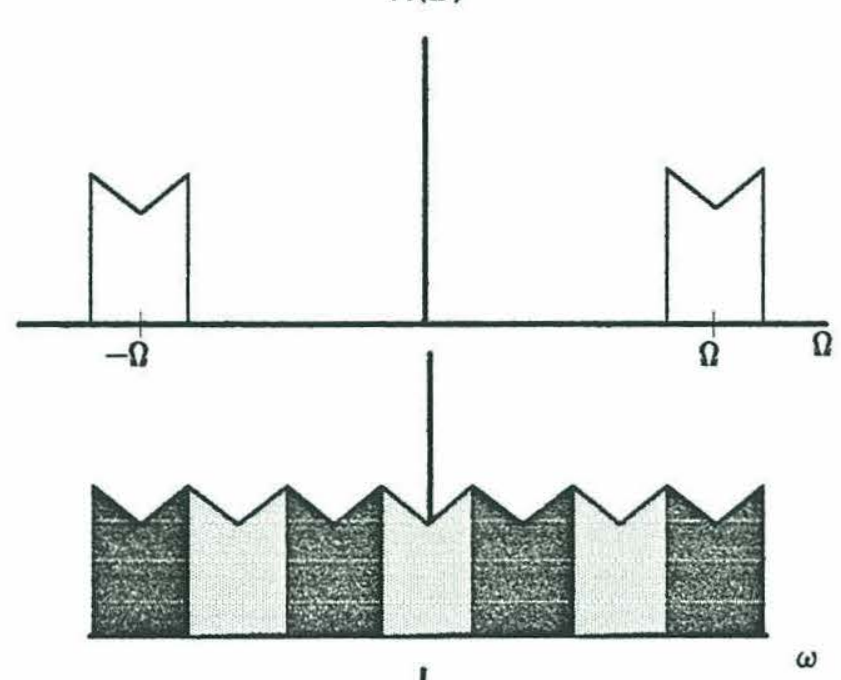

C

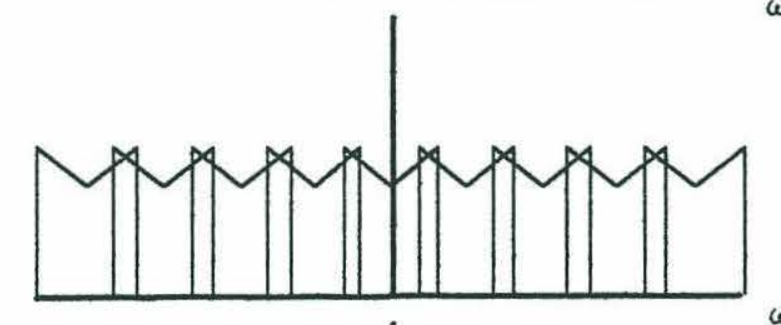

$\omega$

D

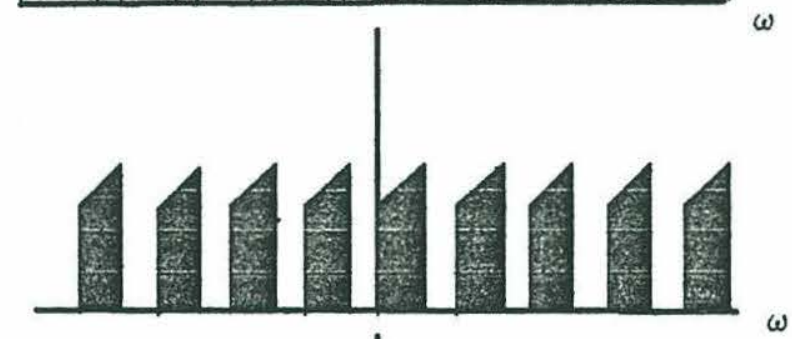

$\mathbf{E}$

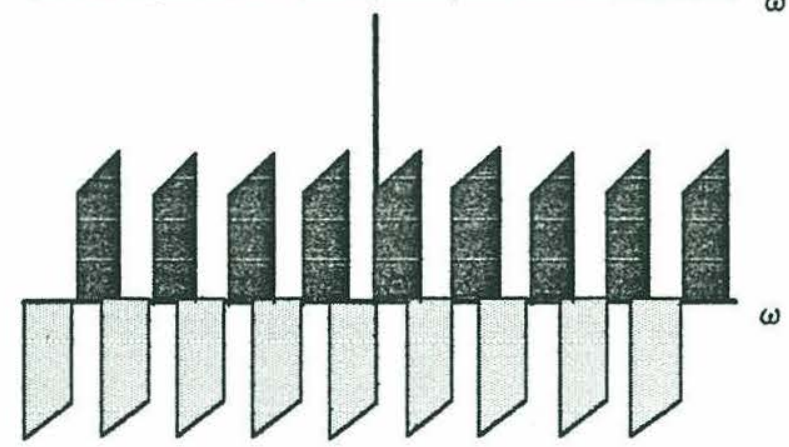

Fig 4.13 Intentional undersampling without aliasing. A purely real signal is illustrated here for convenience but the results can be applied equally well to a complex signal. (A) illustrates the frequency spectrum of the received signal in the continuous time domain. (B) shows the result of sampling this signal at a bandwidth equal to it's envelope. As shown in (C), a further reduction of the sampling rate by ordinary means leads to aliasing. (D) shows only the positive part of the envelope's spectrum, which all information needed to reconstruct the original signal. This portion can be extracted by combining the signal in (C) with the signal in (E), which is produced by sampling the quadrature component of the original signal. 
aliasing will not occur. This could be achieved by a filter with the following frequency response:

$$
\begin{array}{r}
H(\omega)=2 \text { if }|\Omega|>\Omega c \\
0 \text { if }|\Omega|<\Omega c
\end{array}
$$

Equivalently, the signal from Fig $4.13 \mathrm{C}$ could be combined with the response from Fig 4.13E, which represents the response in Fig $4.13 \mathrm{C}$ shifted by $\pi / 4$ radians. This response can be produced by dividing the samples between an in-phase component, $\mathrm{XI}_{\mathrm{I}}(\mathrm{t})$ and a quadrature component, $\mathrm{XQ}_{\mathrm{Q}}(\mathrm{t})$ and combining them [8]:

$$
\mathrm{X}^{+}(\mathrm{t})=\mathrm{XI}(\mathrm{t})-j \mathrm{XQ}(\mathrm{t})
$$
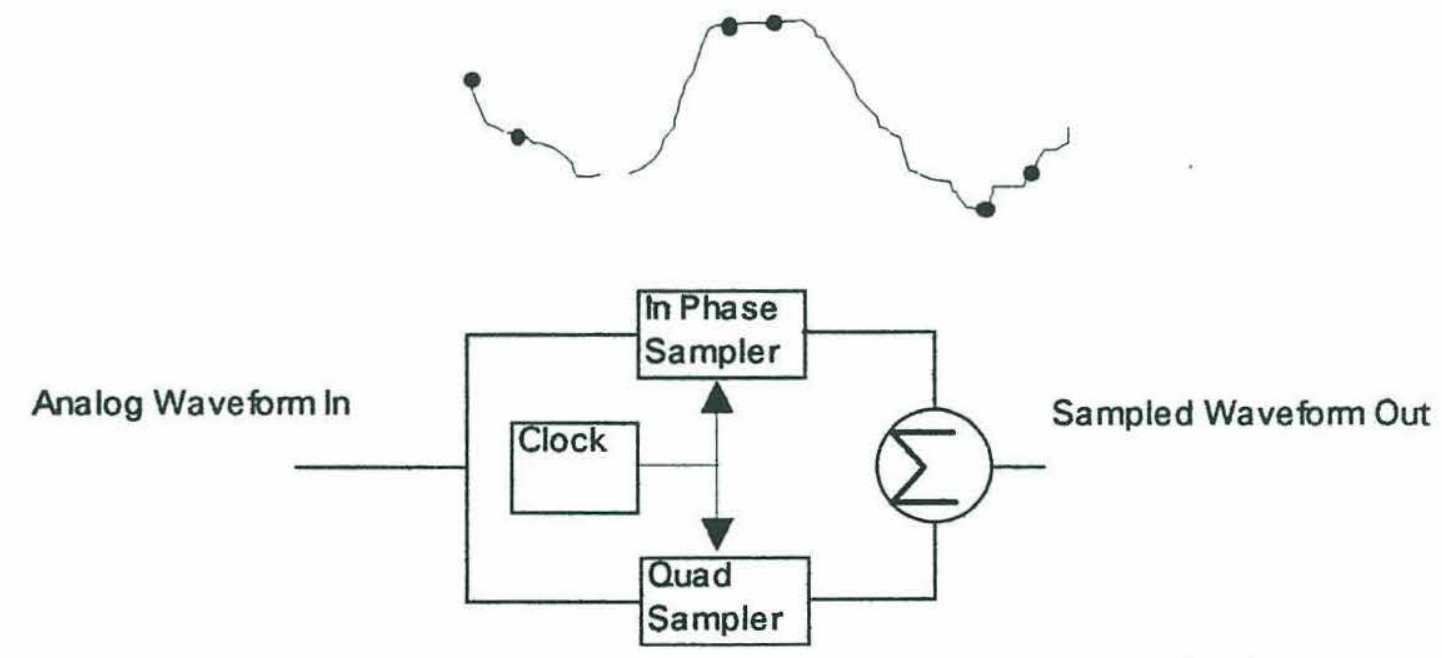

Fig 4.14 Quadrature downsampling scheme. Rather than distributing the samples equally along the waveform, they are organized in complex pairs.

Fig 4.14 illustrates the sampling scheme suggested by equation 4.9. It is implemented by pairing the sample points, each point spaced one quarter cycle from its partner. Thus, the original $300 \mathrm{kHz}$ signal can be effectively sampled at as low a rate as 7.5 
$\mathrm{kHz}$ complex, each complex sample consisting of a point and its quarter cycle partner. This method, known as "delay sampling" or "quadrature downsampling", is used by both the DSL-200 and DSL-300 at $10 \mathrm{kHz}$ complex. The extra samples prevents aliasing due to the side lobes of the Butterworth filter [8].

With the detailed system knowledge presented here, we are now ready to develop a specific mathematical representation, the subject of the next chapter. This representation will concentrate on the DSL-300. 


\section{Chapter 5}

\section{The DSL 300-kHz Sonar Model}

\subsection{Introduction}

Now that the operation of the DSL $200 / 300-\mathrm{kHz}$ sonar systems is understood, it is desirable to translate this knowledge into a mathematical model. This task will be accomplished, for the DSL-300, with the help of the MATLAB (MATrix LABoratory) interactive mathematics software. The MATLAB software environment is uniquely suited to signal processing. It allows complicated operations, such as the Fast Fourier Transform (FFT), to be performed quickly and easily.

The model consists of two basic sections. The transmitter section analytically reconstructs the transmitted pulse. The receiver section consists of a number of blocks, each with a specified frequency and phase response. This section duplicates mathematically the processing of the returned pulse in the actual system, as described in Chapter 4.

Fig 5.1 presents a block diagram of the processing model. Although similar to the system block diagram discussed in Chapter 4 , it is structured to simulate the sequential flow of the signal from pulse generation through processing and digitization of the return.

The model begins with the transmitter, which represents the generation and 


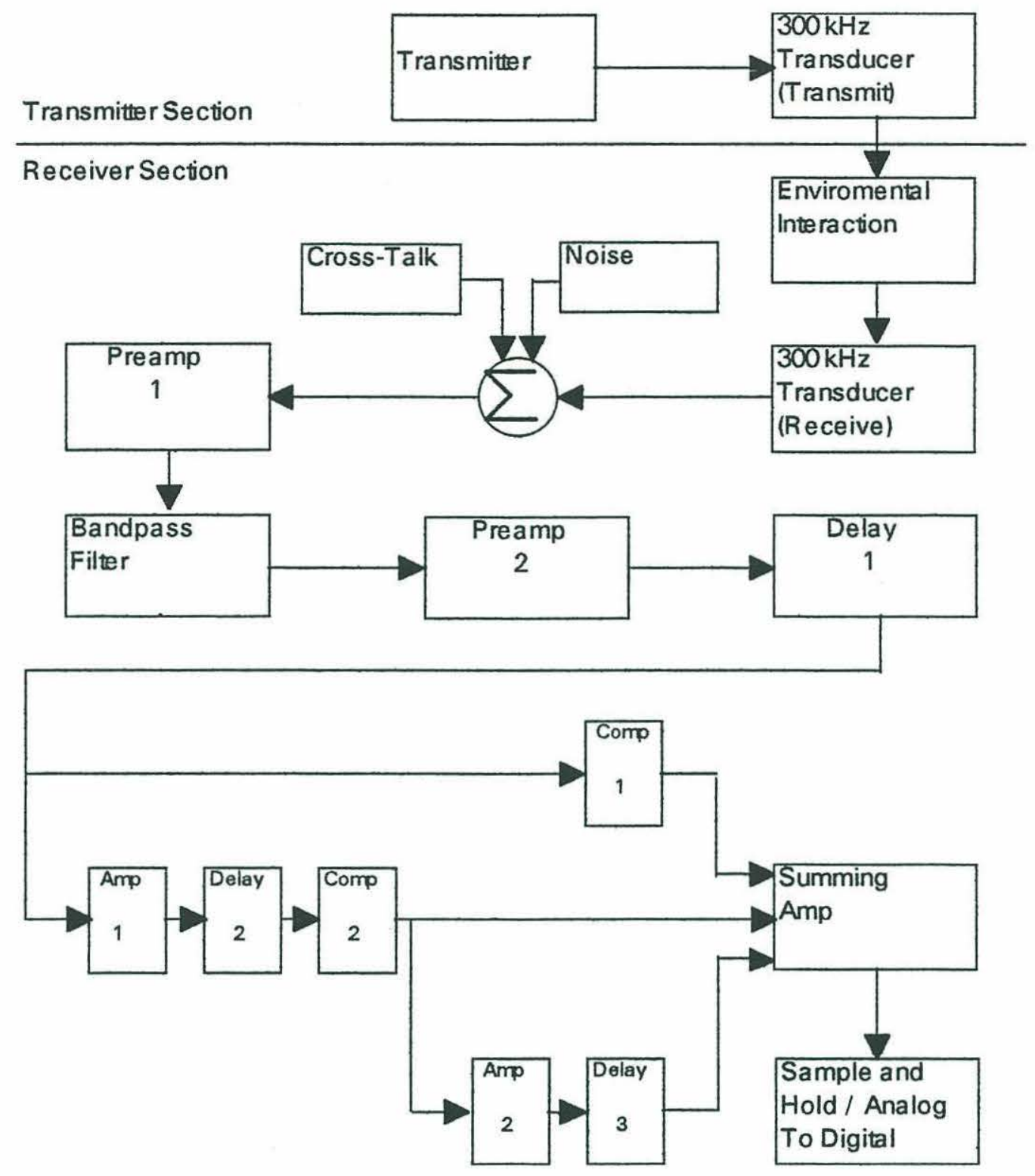

Fig 5.1 Flow chart of the $300-\mathrm{kHz}$ system model.

amplification of the pulse. It is filtered by the transducer and further modified by interaction with the environment. The environmental interaction, in reality a complex process (which is beyond the scope of this paper), will be modeled as a simple attenuation since the concern here is with the response of the sensor itself. The returned pulse will again encounters the transducer, which has a slightly different response when receiving as opposed to transmitting. Following the transducer, the 
signal is combined with amplifier noise and crosstalk from adjacent channels. The combined return is then filtered and amplified in the preamp/bandpass block and time shifted in the delay block (which represents the finite time delay of the amplifiers). The signal now splits into several paths depending on the gain selected. Each path contains a gain element, a delay element, and a phase-compensating element. The separate pathways recombine in the summing amplifier, which is followed by a final delay element. The final stage is the sample and hold, where the signal is prepared for digital representation.

In order to represent the analog waveforms and circuit responses in MATLAB's digital format, the signal must be sampled at a finite rate. At minimum, this must be the Nyquist rate of twice the highest frequency present. If one assumes that the highest frequency of interest is $350 \mathrm{kHz}$, this indicates a sampling rate of $700 \mathrm{kHz}$. In order to allow a smooth time-domain representation of the signal, and to account for the presence of frequencies even higher than $350 \mathrm{kHz}$, a significantly higher sampling rate is used. The rate chosen was $16.384 \mathrm{mHz}$, since this translates to a convenient 8192 samples in the 500-microsecond time window used for the representation. Being a power of two, this allows efficient computation of the discrete fourier transform via the FFT. An even higher number of frequency samples $(32,768)$ were used to enhance resolution of the narrow band signal. Although the number of frequency samples required could have been reduced through the application of a more sophisticated transform technique, the method chosen allows convenient transition between the two domains. These array sizes are well within the capabilities of the SUN workstation used to process in a timely fashion.

For convenience, a standard pulse length of 300 microseconds is used throughout most of this chapter, although the effects of using a short pulse will also be illustrated. 


\subsection{The Transmitter Section}

Ideally, the envelope of the transmitted pulse would be perfectly rectangular. This, of course, is impossible to achieve in any real system, due primarily to the finite bandwidths of the amplifier and transducer. Detailed information about the structure of the real pulse is therefore required.

Since such structural information was not readily available, a test was required. To capture an accurate replica of the transmitted pulse, a hydrophone with a flat frequency response in the band of interest was needed. The best device obtainable was the EDO Model 6600. Its frequency response is illustrated in Fig 5.2. It will be noted that its response is less than ideal in the $300 \mathrm{kHz}$ range. Fortunately, this turns out not to have a serious corrupting effect on the pulse, as discussed below.

The experiment was conducted in June 1992, dockside at the Woods Hole Oceanographic Institution. The EDO hydrophone was mounted on a wooden support fastened to the DSL sonar sled, so that it was positioned five feet from the transmitter face (Fig 5.3). Exact positioning of the device was not crucial, since the object was to obtain information about the pulse shape; data regarding source levels and beam patterns was already available from manufacturers tests. No amplification of the hydrophone output was required due to the high source level of the DSL 300 . Using the 300-microsecond pulse length, the output was captured on a Textronics Model 2232 oscilloscope and photographed with a Polaroid camera. The recorded pulse envelope is illustrated in Fig 5.4. 


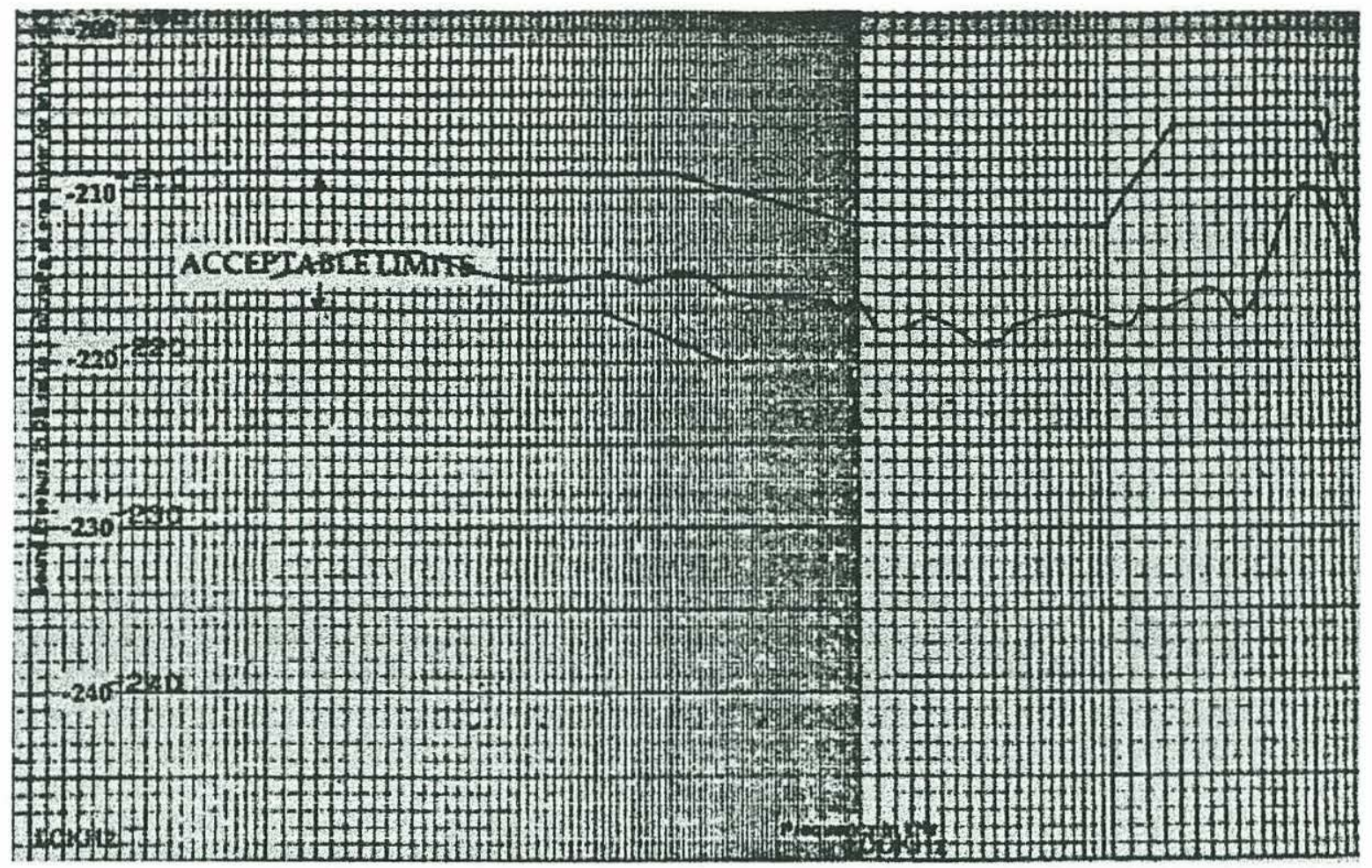

Fig 5.2 Measured frequency response of the EDO transducer.

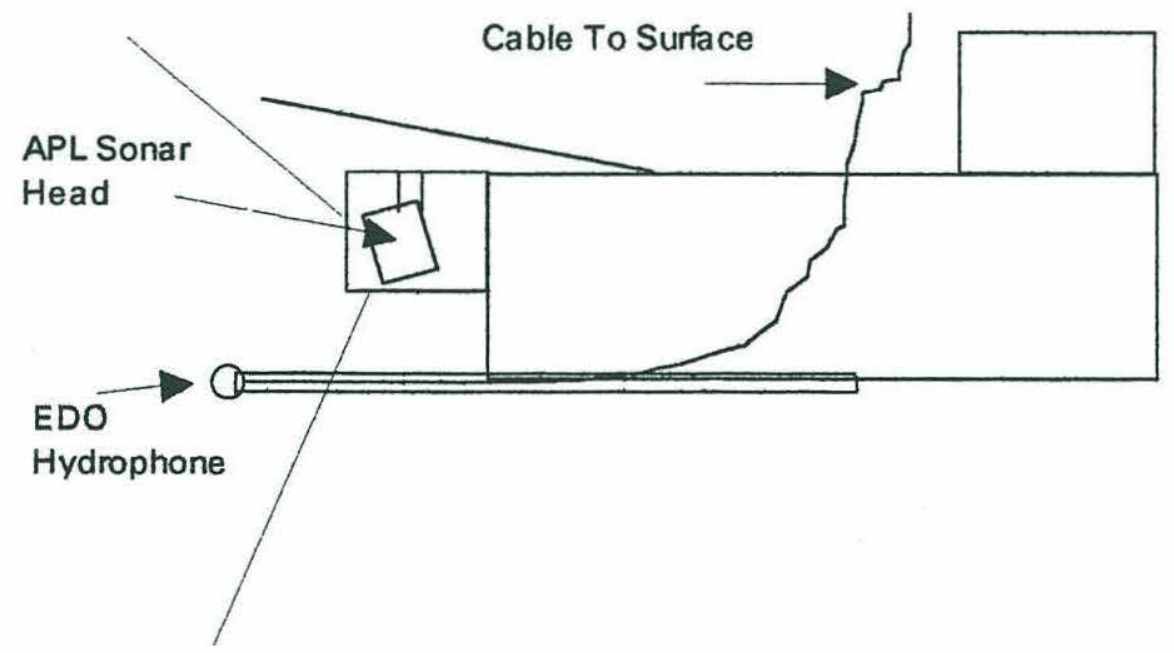

Fig 5.3 Experimental setup for determining pulse characteristics. 


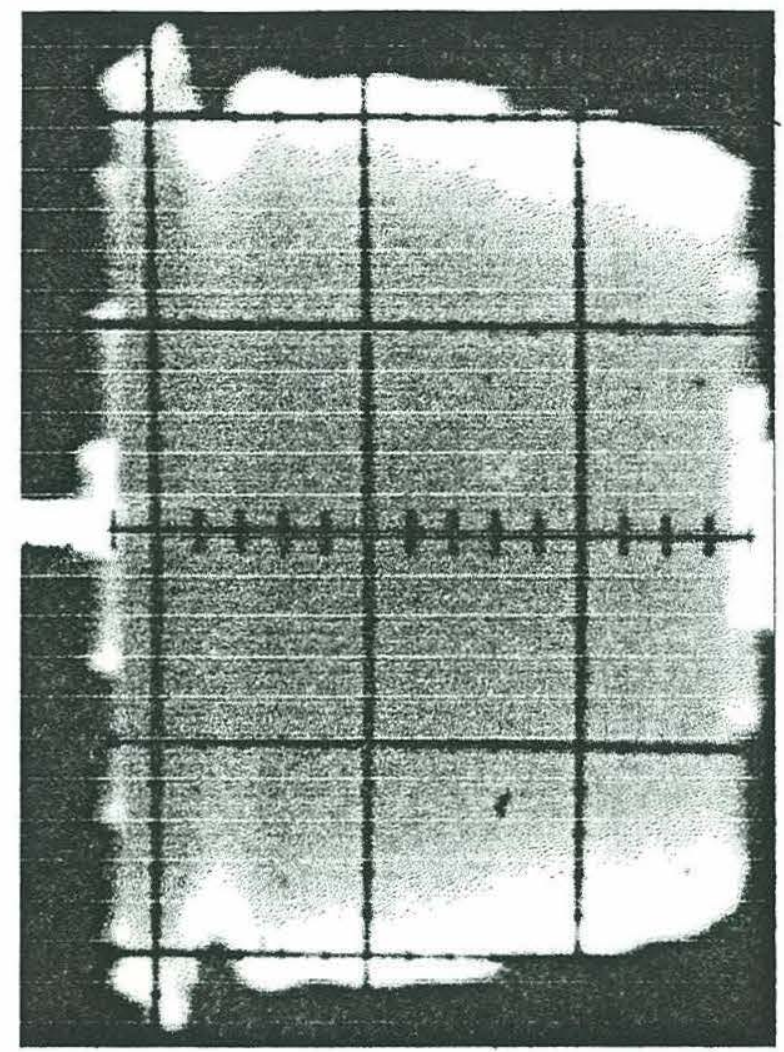

Fig 5.4 Photograph of the $300-\mathrm{kHz}$ pulse, as recorded by the EDO hydrophone. Time scale is 100 microseconds per division. Vertical scale is $200 \mathrm{mV}$ per division.

The first module depicted in Fig 5.1 is the transmitter. This block combines the functions of pulse generation and amplification. Assuming that the coupling transformer is ideal, the signal leaving this stage will be characterized as a simple rectangular pulse. This is because the turn-on time of the IRFP250 MOSFET is approximately $100 \mu \mathrm{sec}$, which is small when compared to the pulse length (departure of the transformer from the ideal is discussed below). This is represented mathematically as:

$$
\begin{array}{rlrl}
h_{w}(t) & =G_{a} & t b & \leq t \leq \text { tend } \\
& =0 & & \text { otherwise }
\end{array}
$$


where $h_{w}$ is the time envelope of the pulse, $G_{a}$ is the amplifier gain, to is the beginning time, and tend is the ending time. The carrier is represented by a complex exponential:

$$
V_{c}(t)=e^{(2 \pi f c)} \text { volts }
$$

Where $\mathrm{fc}_{\mathrm{c}}$ is the carrier frequency, $300 \mathrm{kHz}$ in this case. Therefore the pulse leaving the power transmitter is given by

$$
V_{p}(t)=h_{w}(t) V_{c}(t) \text { volts }
$$

This is illustrated in Fig 5.5 for $\mathrm{Ga}_{\mathrm{a}}=680$, tb $=100 \mu \mathrm{s}$, tend $=400 \mu \mathrm{s}$, and $\mathrm{f}_{\mathrm{c}}=300$ $\mathrm{kHz}$.

The amplified pulse now enters the transducer, which behaves as a filter in terms of the model. Fig 3.4 illustrates an equivalent circuit for a transducer as a series RLC in parallel with a shunt resistance and a shunt capacitance. The model can be considered in terms of two parallel admittances: the combined admittance of shunt elements Co and Ro is called the "blocking" admittance, while the series elements R, L, and C form what is called the "motional" admittance [17]. R actually consists of two parts:

$$
R=R_{R}+R_{L}
$$

$R_{R}$ represents the radiation resistance and is associated with power actually transmitted into the water, while $\mathrm{RL}$ represents losses due to internal heating and mounting [9].

In order to properly characterize the transducer, equivalent values of $\mathrm{R}, \mathrm{L}$, and $\mathrm{C}$ 


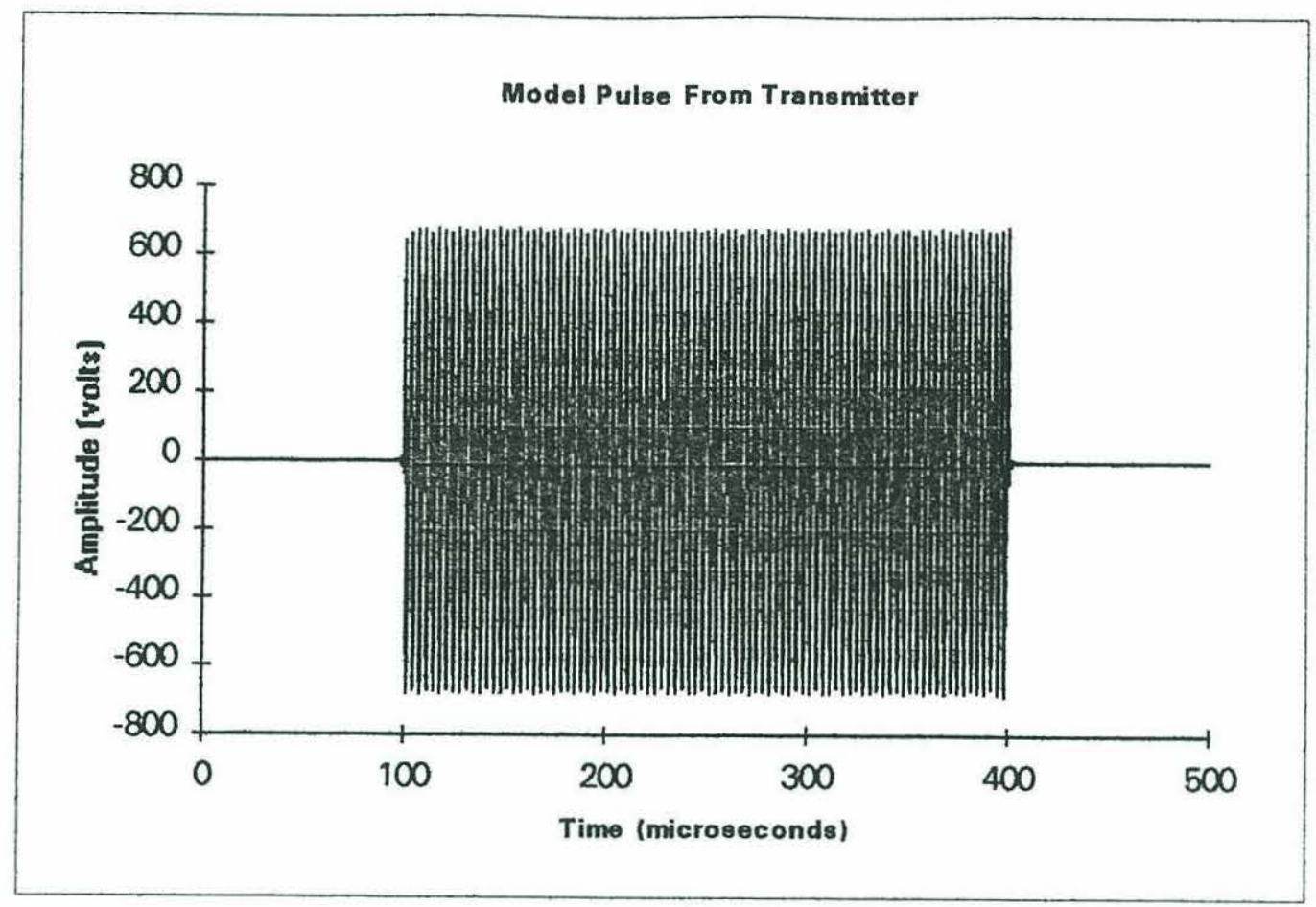

Fig 5.5 Model pulse before entering the transducer.

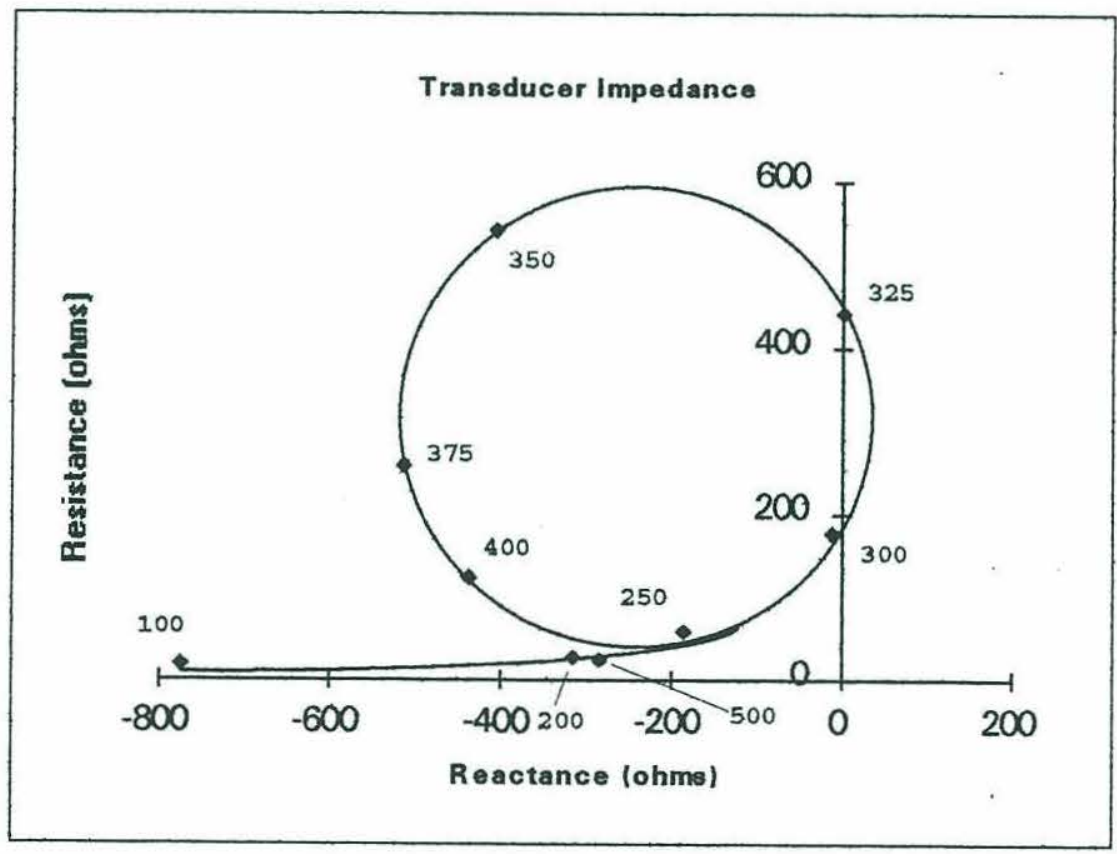

Fig 5.6 Complex impedance plot of the $300-\mathrm{kHz}$ transducer. (Small number indicate frequency in $\mathrm{kHz}$ ). 


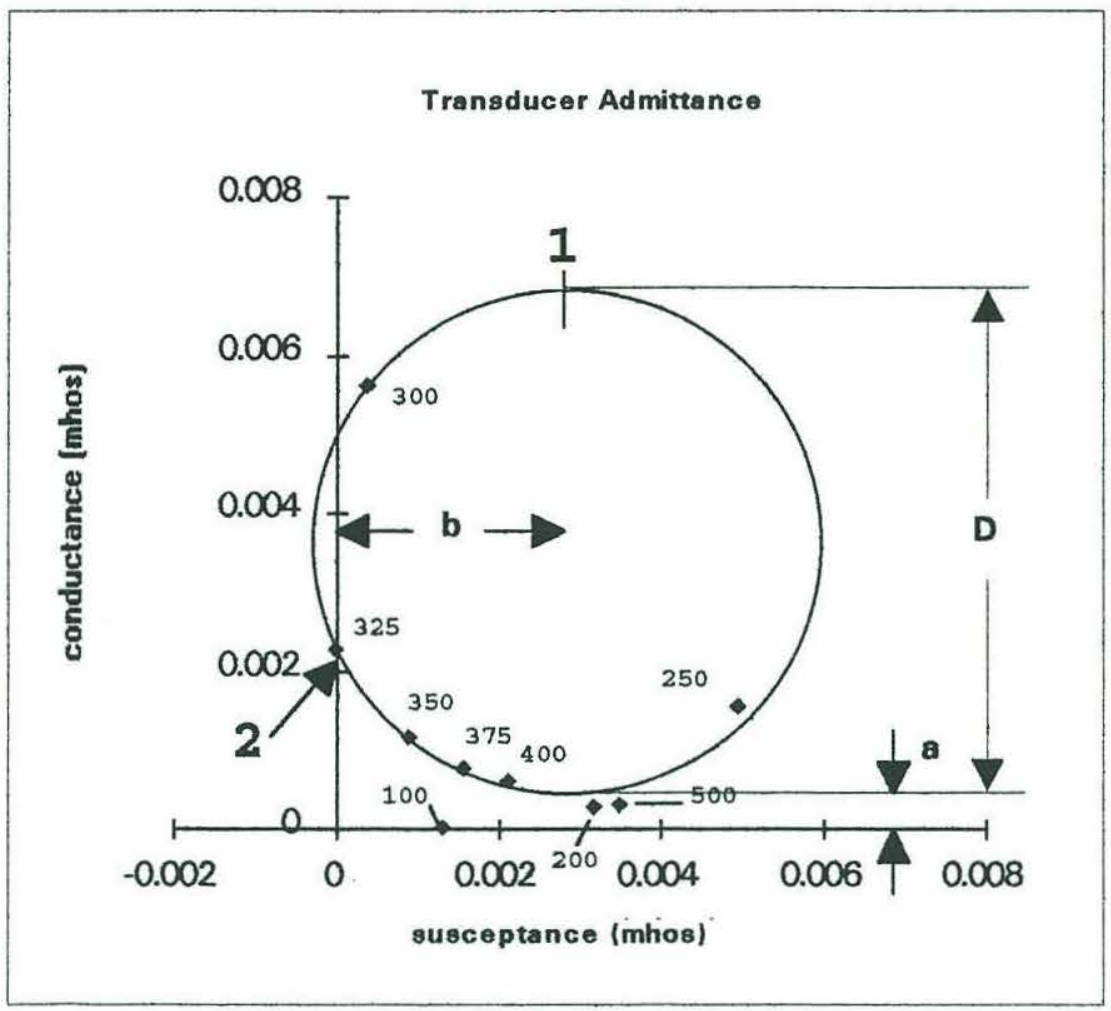

Fig 5.7 Data from Fig 5.6, replotted in terms of admittance (small numbers are frequency in $\mathrm{kHz}$ ).

must be assigned. This can be accomplished with the help of the complex impedance plot shown in Fig 5.6, which was produced at APL. Since it is more convenient to analyze this circuit in terms of admittances, the data points are replotted on a complex admittance chart in Fig 5.7. Here, an idealized circle has been superimposed on the diagram. It can be shown that the diameter of this circle is a measure of the real resistance of the motional branch:

$$
\mathrm{D}=\frac{1}{\mathrm{R}}
$$

while the distance from the imaginary axis to the circle represents the blocked admittance: 


$$
\mathrm{a}=\frac{1}{\mathrm{Ro}}
$$

The displacement of the center of the circle from the real axis determines the value of the blocked capacitance [9]:

$$
\mathrm{C}=\omega_{\mathrm{ob}}
$$

where $\omega_{0}$ is the resonant frequency. Since the transmit value of $\mathrm{Q}$ is known from the frequency-response curve discussed in Chapter 4, it is possible to determine $\mathrm{L}$ from:

$$
\mathrm{L}=\frac{\mathrm{QR}}{\omega_{0}}
$$

Once $\mathrm{L}$ is known, $\mathrm{C}$ is also:

$$
C=\frac{1}{\omega_{0}^{2} L}
$$

\begin{tabular}{|llllll|}
\hline $\mathbf{R} R$ & $\mathbf{R} \mathbf{L}$ & $\mathbf{L}$ & $\mathbf{C}$ & $\mathbf{R}_{\mathbf{0}}$ & $\mathbf{C}_{0}$ \\
$63.8 \Omega$ & $97.5 \Omega$ & $0.753 \mathrm{mH}$ & $363.8 \mathrm{pF}$ & $3.1 \mathrm{~K}$ & $3,610.0 \mathrm{pF}$ \\
\hline
\end{tabular}

Table 5.1 Component values for model transducer. $\mathrm{R} 0$ and $\mathrm{C} 0$ represent the intrinsic shunt resistance and capacitance combined with discrete shunt elements from the circuit. $R R$ and $R R$ were determined from $R$ and the calculated transducer efficiency of $39.6 \%$.

$\mathrm{RR}$ and $\mathrm{RL}$ can be determined from the calculated value of $\mathrm{R}$ and the transducer efficiency. The efficiency is determined by comparing the measured transmitted power from Chapter 4 with the average power of the model pulse. This calculation has been done in Table 5.1 by anticipating the results of this section. Table 5.1 also lists the values of $\mathrm{R}_{\mathrm{o}}, \mathrm{C}, \mathrm{C}_{\mathrm{o}}$ and $\mathrm{L}$ as determined from Fig 5.7. 
When modeling the transducer response for the transmitted pulse, it is possible to ignore Ro and Co and use a simple oscillator, as shown in Fig 5.8. This is because the shunt elements combine with the series leakage reactance of the transformer to form a filter with unity gain near $300 \mathrm{kHz}$ (this filter will have some effect of the transmitted pulse, as described below). This allows the use of the transmit response curve to calculate the equivalent circuit elements, since this plot represents only the mechanical elements. When used to transmit, the transducer operates at point 1 on the admittance circle, as shown in Fig 5.7 [9].

From Fig 5.8, the frequency response of the transmitting transducer, $\mathrm{H}_{u}$, is easily calculated:

$$
\mathrm{Ht}(\omega)=\frac{\mathrm{R}}{\mathrm{R}+j \omega \mathrm{L}-\frac{j}{\omega \mathrm{C}}}
$$

Rearranging and substituting the known values yields:

$$
\mathrm{H}_{\mathrm{t}}(\mathrm{f})=\frac{\mathrm{a}_{2} \mathrm{f}^{2}+j\left(\mathrm{~b}_{3} \mathrm{f}^{3}+\mathrm{b}_{1} f\right)}{\mathrm{c}_{4} \mathrm{f}^{4}+\mathrm{c}_{2} \mathrm{f}^{2}+\mathrm{co}_{\mathrm{o}}}
$$

where the frequency $\mathrm{f}$ is expressed in $\mathrm{kHz}$. This can be put into polar form:

$$
H_{t(\text { mag })}(f)=\sqrt{\frac{a 6 f^{6}+a 4 f^{4}+a 2 f^{2}}{c 8 f^{8}+c 6 f^{6}+c 4 f^{4}+c 2 f^{2}+c 0}}
$$




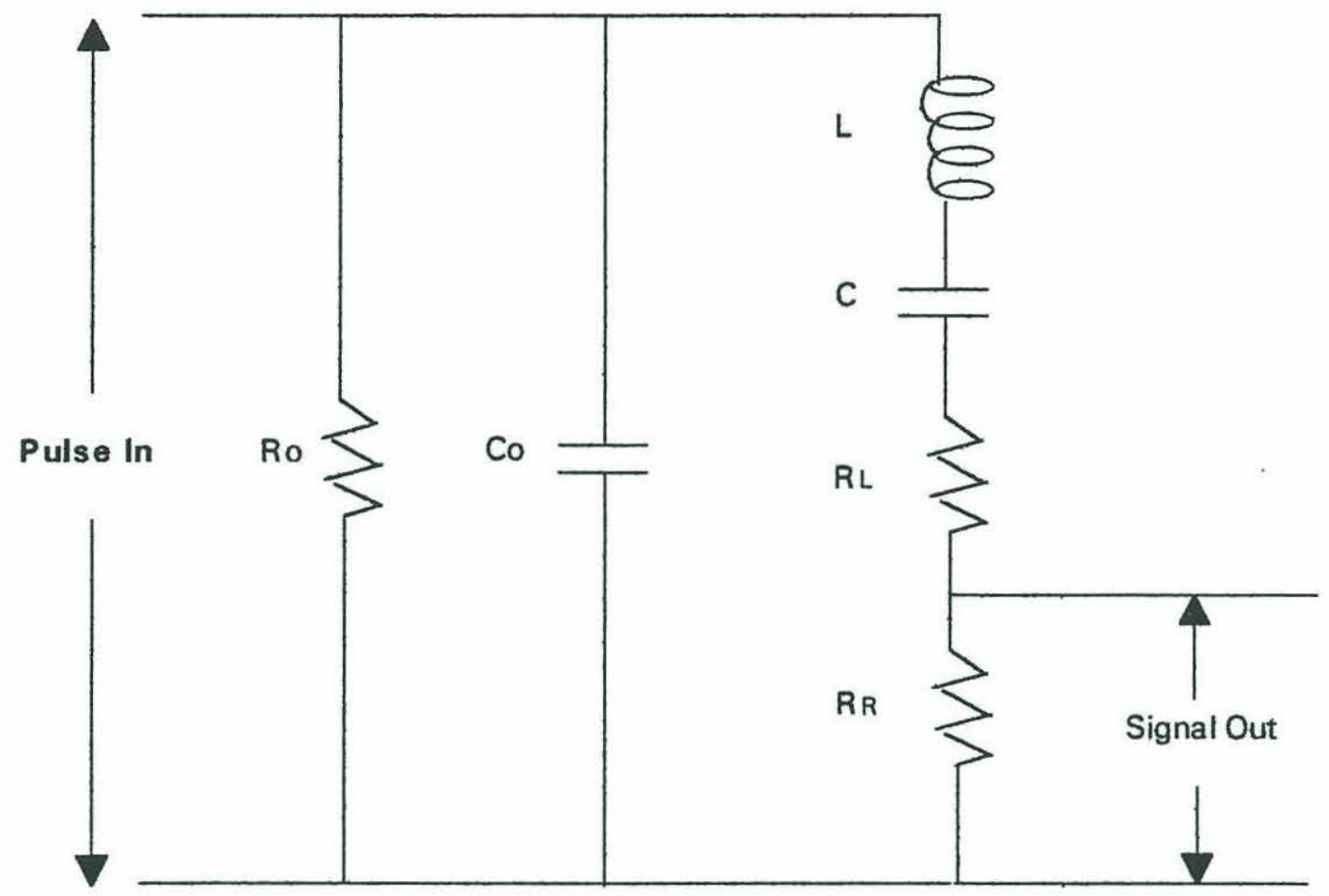

Fig 5.8 The transducer, when acting as a transmitter, can be represented as a simple RLC oscillator.

$$
H_{\text {tt(phase })}(f)=\tan ^{-1}\left(\frac{\text { aif }+a 3 f^{3}}{f^{2}}\right)
$$

A list of coefficients for each of these equations is given by Table 5.2. These results are plotted in Figs 5.9 and 5.10. Convolving $h_{u}$, the impulse response of $\mathrm{H}_{\mathrm{tt}}$, with $\mathrm{V}_{\mathrm{p}}$ and converting to disipated power, $\mathrm{P}_{\mathrm{p}}$, yields:

$$
P_{p}(t)=\frac{\left(B(0) V_{p}(t) * h_{t t}(t)\right)^{2}}{R} \text { Watts }
$$

where $\mathrm{B}(0)$ is the on-axis beam pattern (assumed to be one in this case). The impulse response is illustrated in Fig 5.11, while Fig 5.12 shows the transmitted pulse in terms of equivalent voltage. Fig 5.13 illustrates the transmitted power envelope from Equation 5.13. 


\section{Equation 5.11 Equation 5.12a $\quad$ Equation 5.12b}

a0

a1

a2

a3

a4

a5

a6

b0

b1

$2.5 \times 10^{3}$

b2

b3

c0

c1

c2

$-146.7$

$-2.7 \times 10^{-6}$

1.0

c3

c4

$8.2 \times 10^{-4}$

c5

c6

c7

c8

$-2.7 \times 10^{-2}$

$6.7 \times 10^{6}$

$6.2 \times 10^{16}$

$8.6 \times 10^{9}$

-

$-0.027$

$-1.8 \times 10^{-5}$

1.0

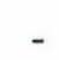

$2.5 \times 10^{3}$

(1.0

-

\section{$4.5 \times 10^{7}$}

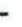

$-330.0$

$9.2 \times 10^{-4}$ 


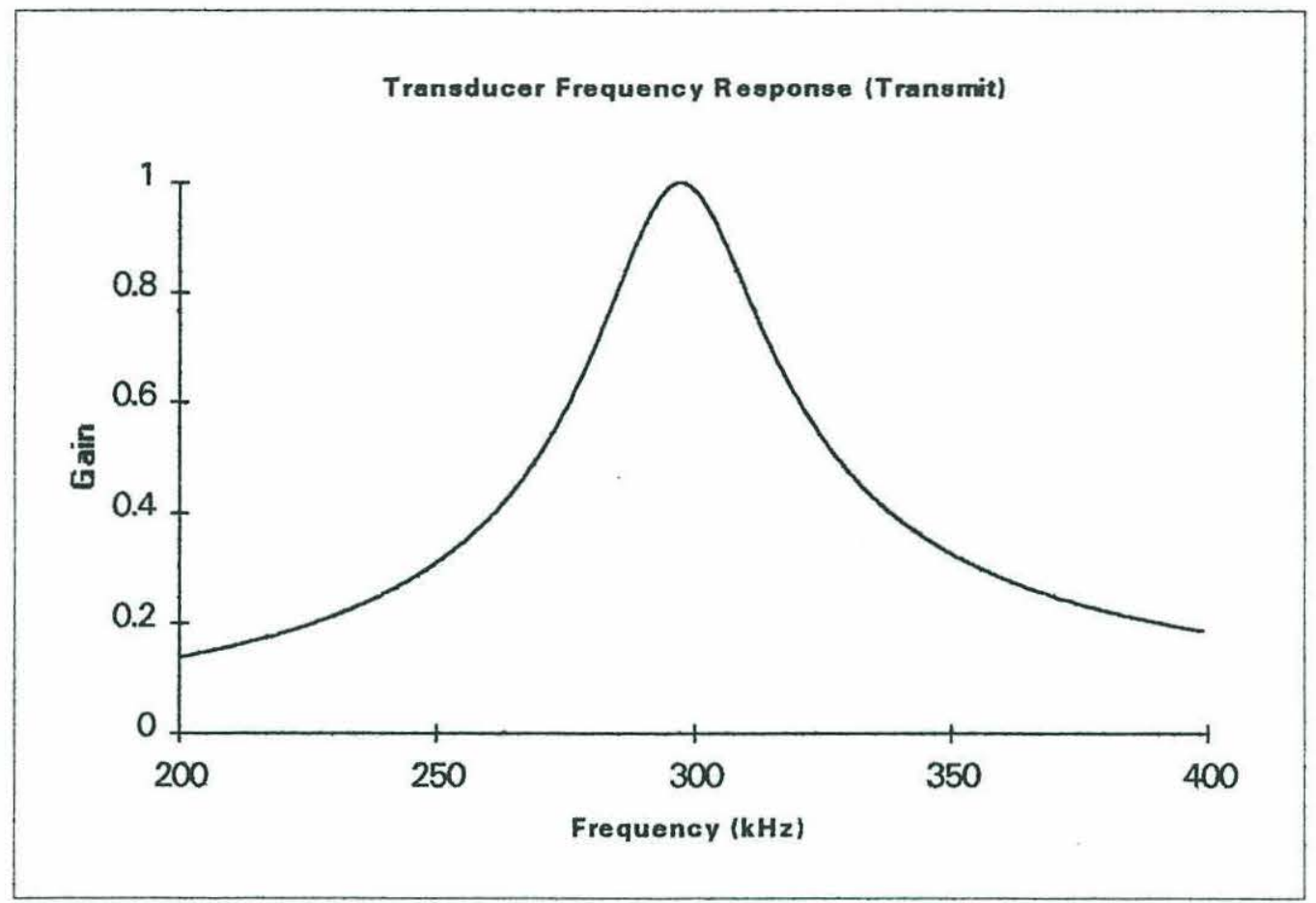

Fig 5.9 Frequency response of the transducer acting as a transmitter.

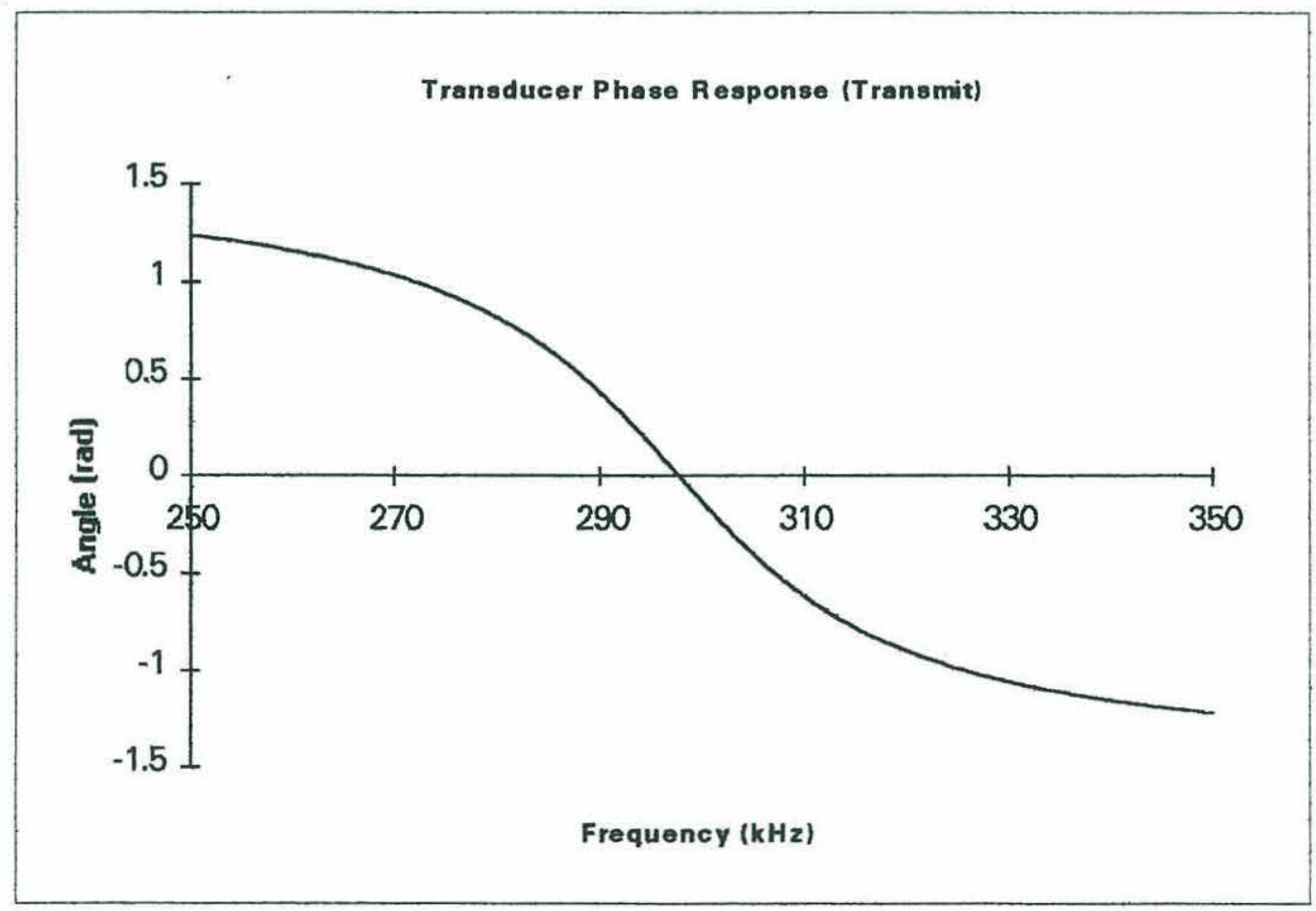

Fig 5.10 Phase response of the transducer acting as a transmitter. 


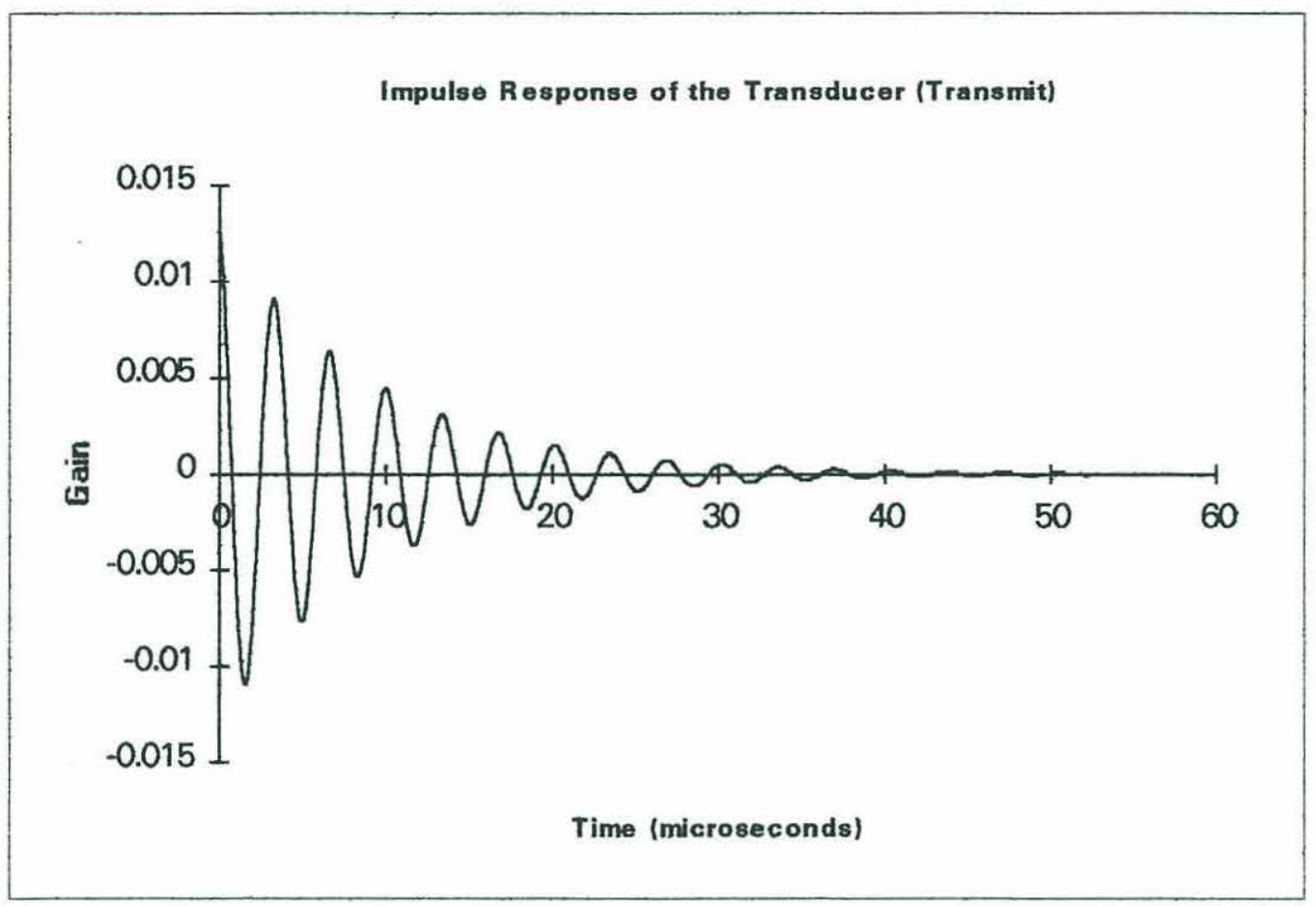

Fig 5.11 Impulse response of the transducer, when used as a transmitter.

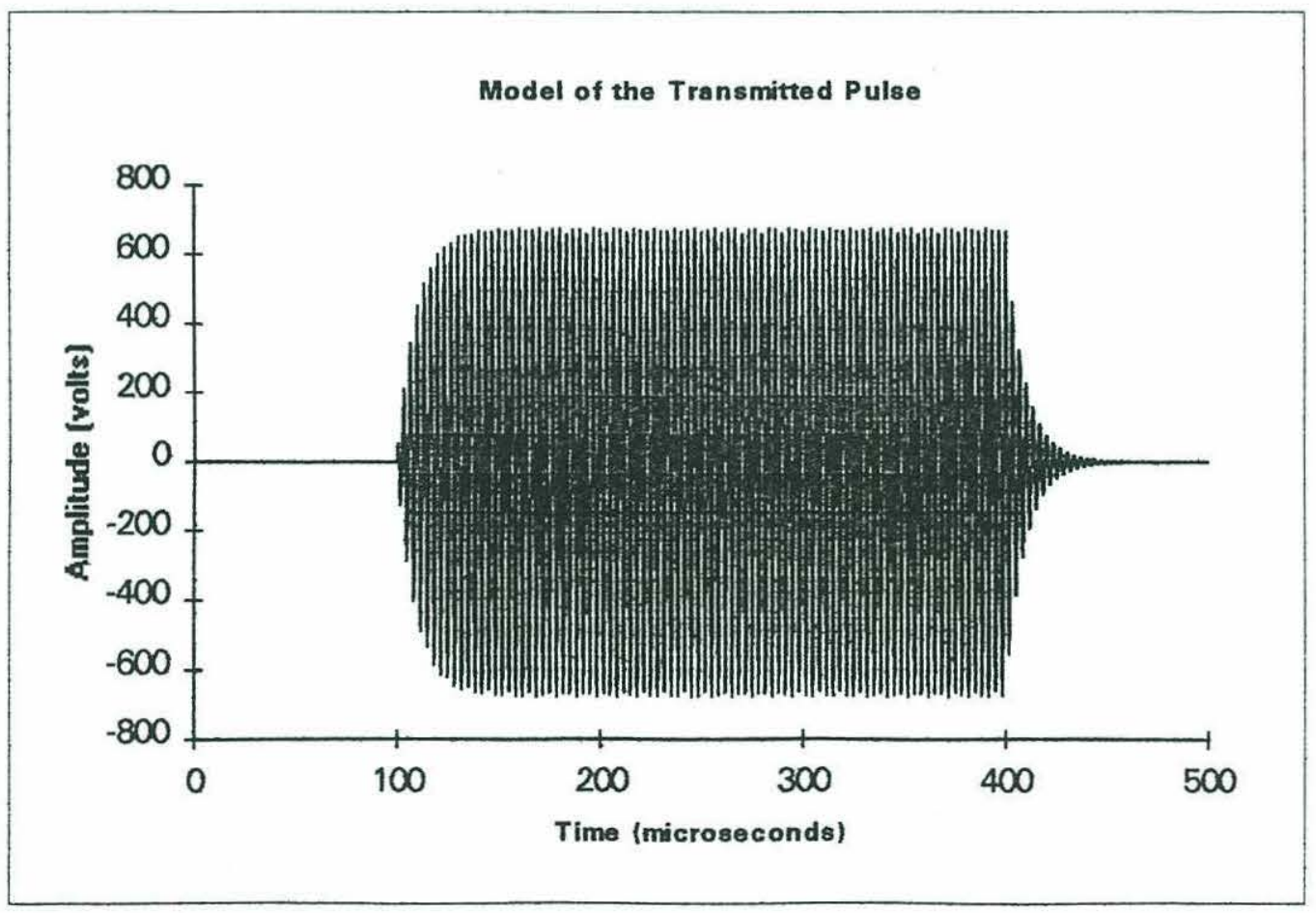

Fig 5.12 Model of the transmitted pulse. 


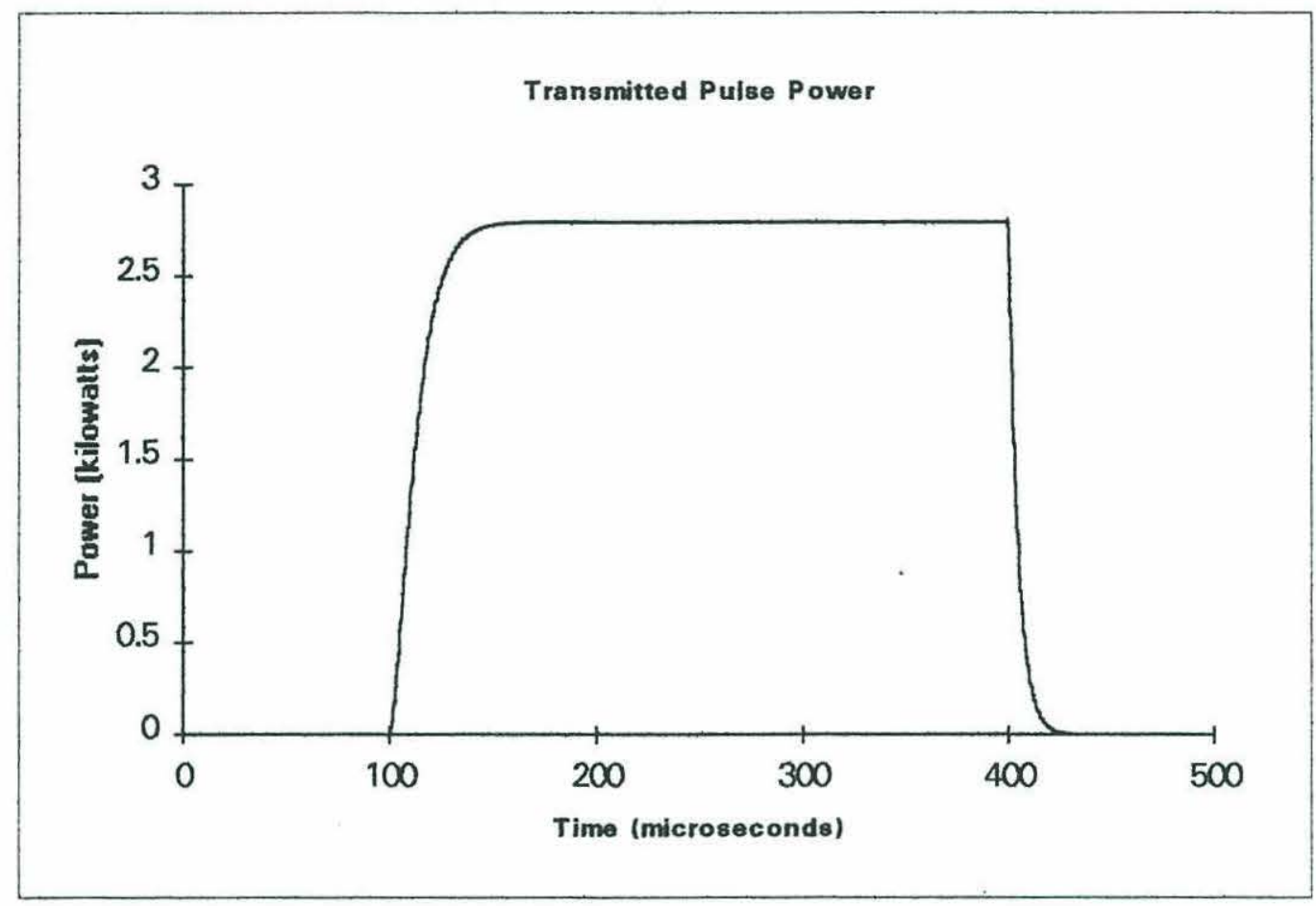

Fig 5.13 Peak power envelope of the transmitted pulse.

Comparing Fig 5.12 to the actual transmitted pulse in Fig 5.4, two significant discrepancies are evident. The first is the decaying oscillation imposed on the pulse envelope at the beginning of the transmit cycle. This oscillation has a frequency of about $20 \mathrm{kHz}$, and represents the beat frequency produced when the high-Q-filter produced by inductor leakage reactance and the blocking branch is excited by the pulse edges. This phenomena will be dealt with by adding an appropriate oscillation term to Equation 5.3. The second discrepancy is the overall drop in pulse amplitude from beginning to end. This is caused by the discharge of the large capacitors in the power supply, which provide the large amounts of required current. The droop is about $18 \%$ for the 300-microsecond pulse and is represented here by an exponential factor. The modified pulse voltage, $V_{\mathrm{pm}}$, is given by: 


$$
\begin{gathered}
\mathrm{V}_{\mathrm{pn}}(\mathrm{t})=\left[\mathrm{V}_{\mathrm{p}}+\mathrm{A} \sin \left(2 \pi \mathrm{f}_{\mathrm{p} t}+\pi / 4\right) \mathrm{e}^{(-\mathrm{t}+\mathrm{tb} / \tau \mathrm{o})}\right] \mathrm{e}^{(-\mathrm{t}+\mathrm{tb}) / \tau \mathrm{d}} \\
\text { for } \mathrm{t}>\mathrm{tb}
\end{gathered}
$$

where $f_{p}$ is the frequency of the parasitic oscillation $(320 \mathrm{kHz})$ with amplitude $\mathrm{A}$, to is the pulse start time, $\tau_{\mathrm{o}}$ is a time constant for the decay of the oscillation ( $\left.20 \mu \mathrm{sec}\right)$, and $\tau \mathrm{d}$ is a time constant associated with the voltage droop $(50000 \mu \mathrm{sec})$. A will initially be set to 250 volts, which is required to produce the proper amplitude after filtering by the transducer. The phase factor of $\pi / 4$ is required to match the observed pulse characteristics.

The final transmitted pulse power is once again given by Equation 5.13 , using $\mathrm{V}_{\mathrm{pn}}$ from 5.16. Fig 5.14 portrays the final time domain pulse in terms of transducer voltage, and Fig 5.15 represents the frequency spectrum.

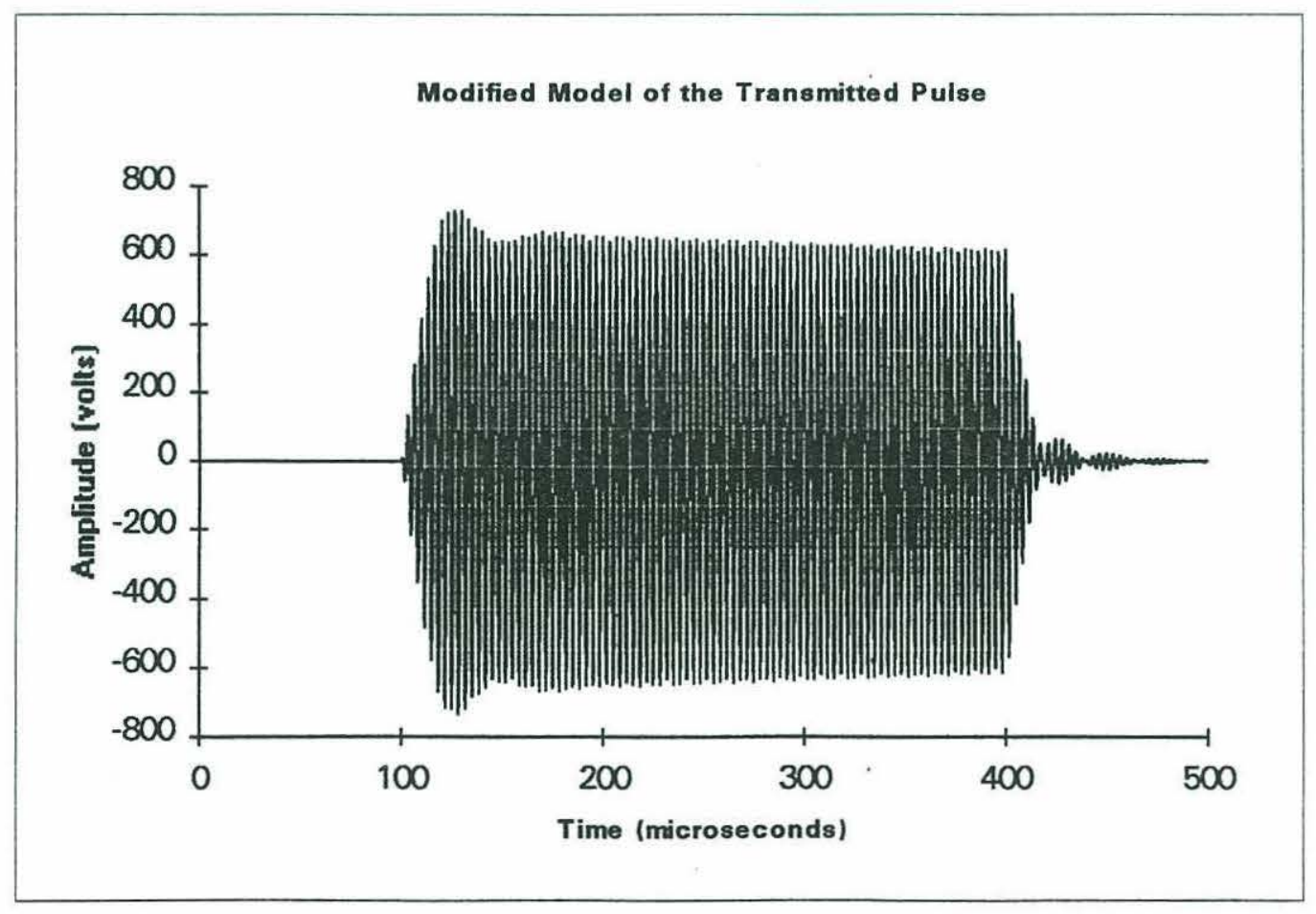

Fig 5.14 Modified transmitted pulse. 


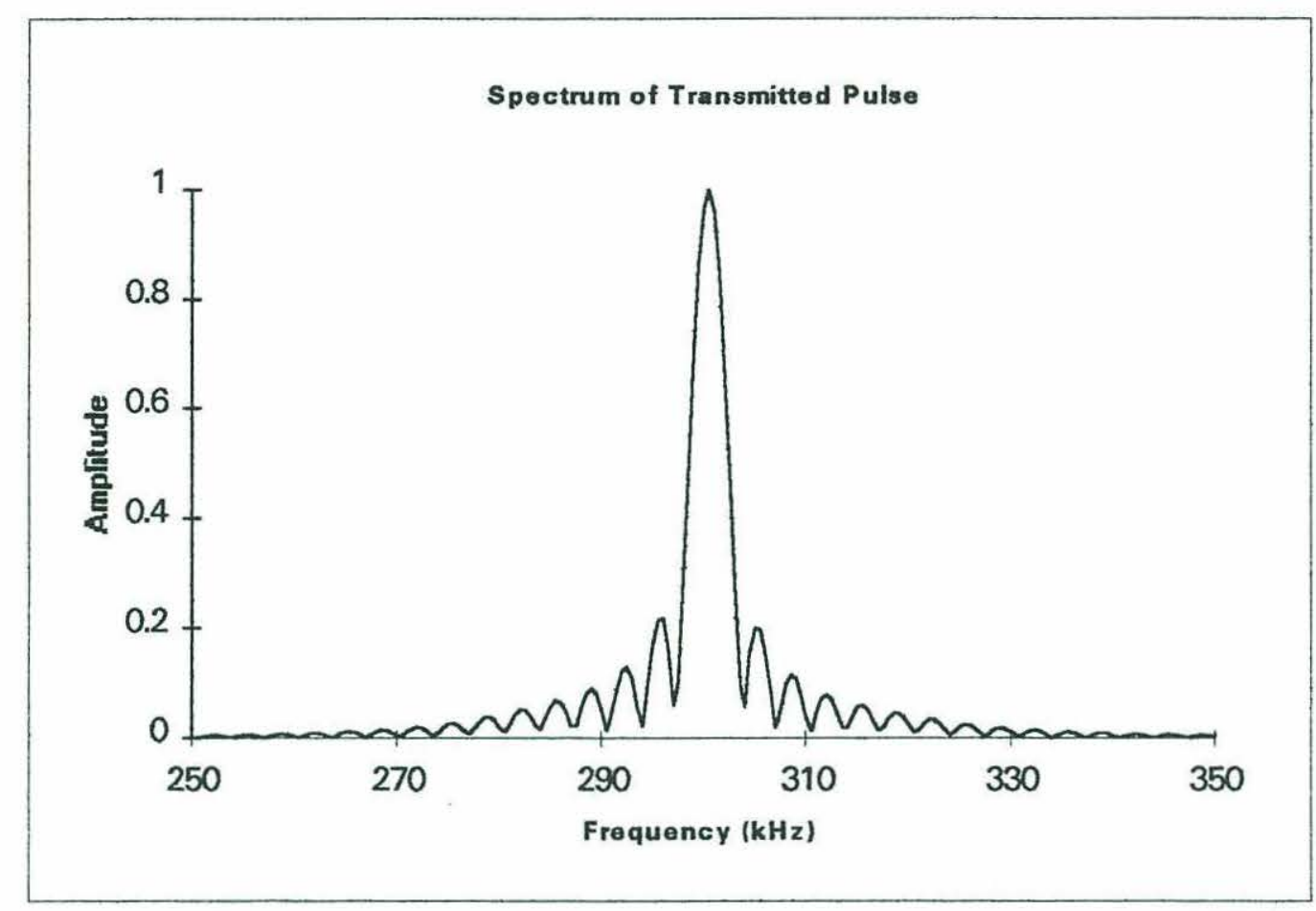

Fig 5.15 Normalized spectrum of the transmitted pulse from Fig 5.15.

It was mentioned earlier that the hydrophone used to measure the transmitted pulse had a less than optimum frequency response near $300 \mathrm{kHz}$. To determine if this causes any noticeable corruption of the pulse, $V_{\text {pulso }}$ was filtered by a linear approximation to the inverse frequency response of the hydrophone, as illustrated in Fig 5.16. Fig 5.17, which shows the result, is seen to have a slightly larger overshoot when compared to Fig 5.14. This is dealt with by raising the amplitude $\mathrm{A}$ of the parasitic oscillation to 400 volts. Fig 5.18 shows the power envelope of the uncorrupted pulse.

Fig 5.19 and 5.20 illustrate the result of selecting the short $(100 \mu \mathrm{sec})$ pulse length.

The pulse now undergoes complex environmental interaction which is not modeled here. The returned signal is represented in the model as a simple square pulse with a 


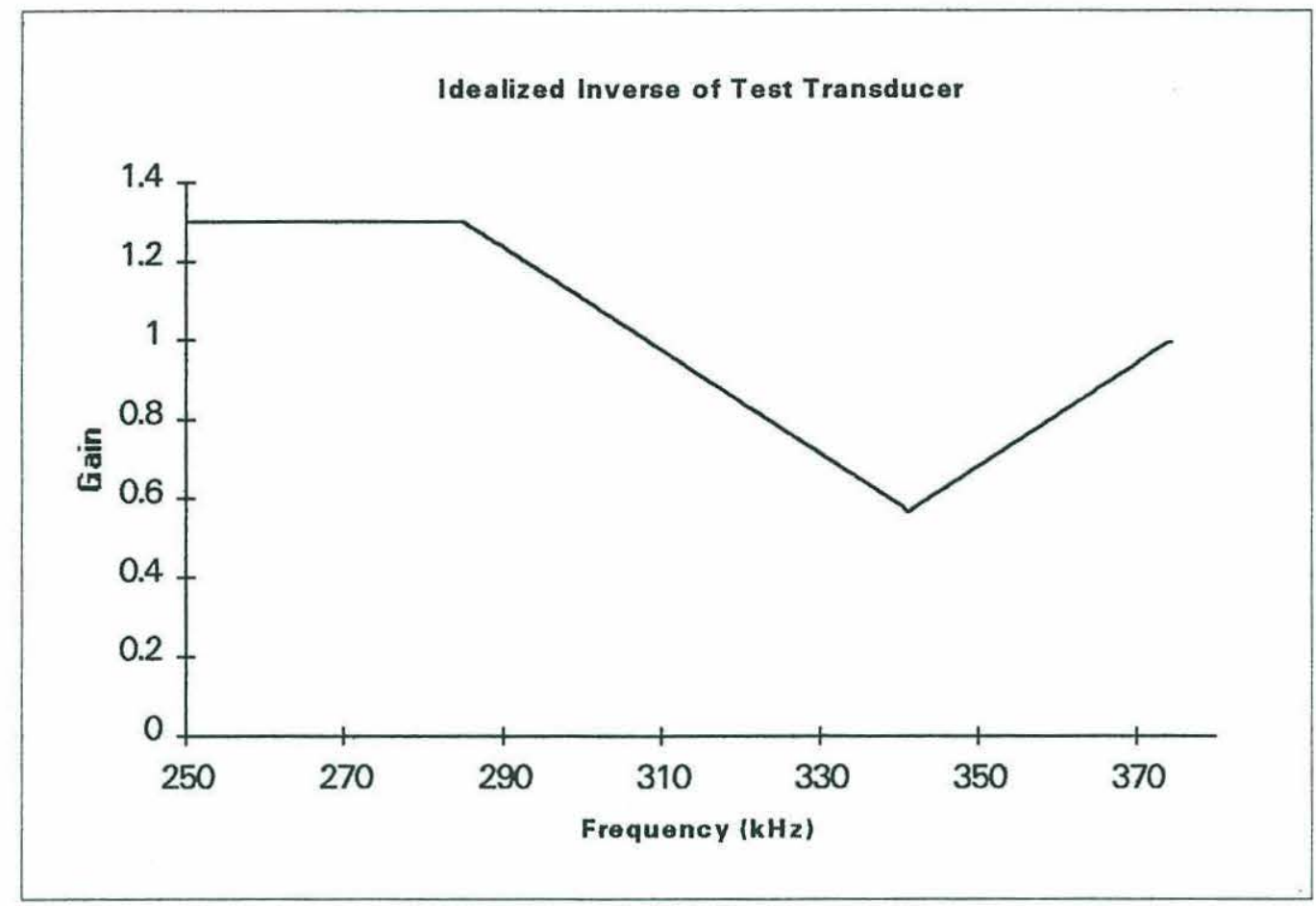

Fig 5.16 An idealized filter to remove the corrupting effects of the EDO hydrophone.

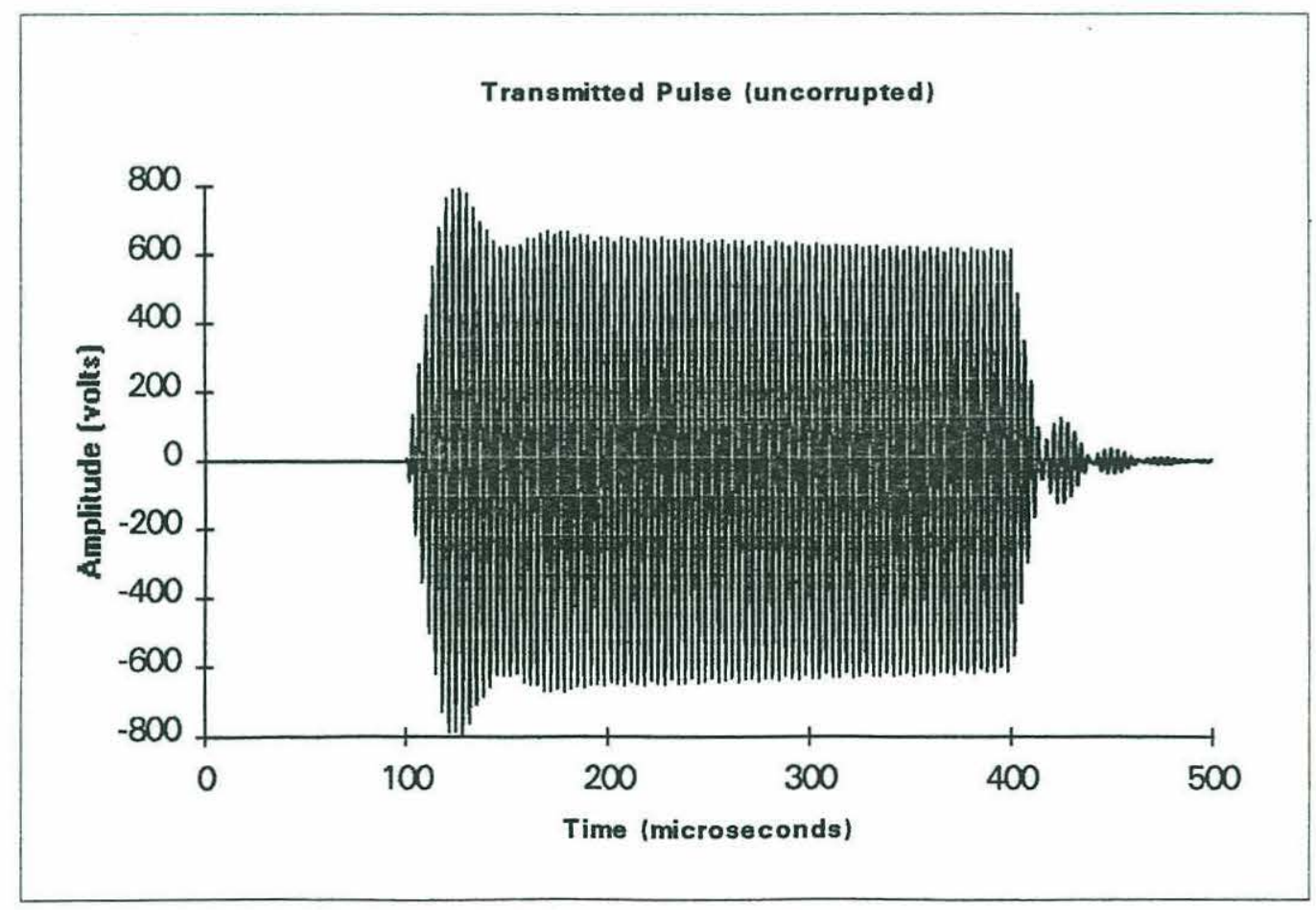

Fig 5.17 Final version of the transmitted pulse, after correction for receiving hydrophone effects. 


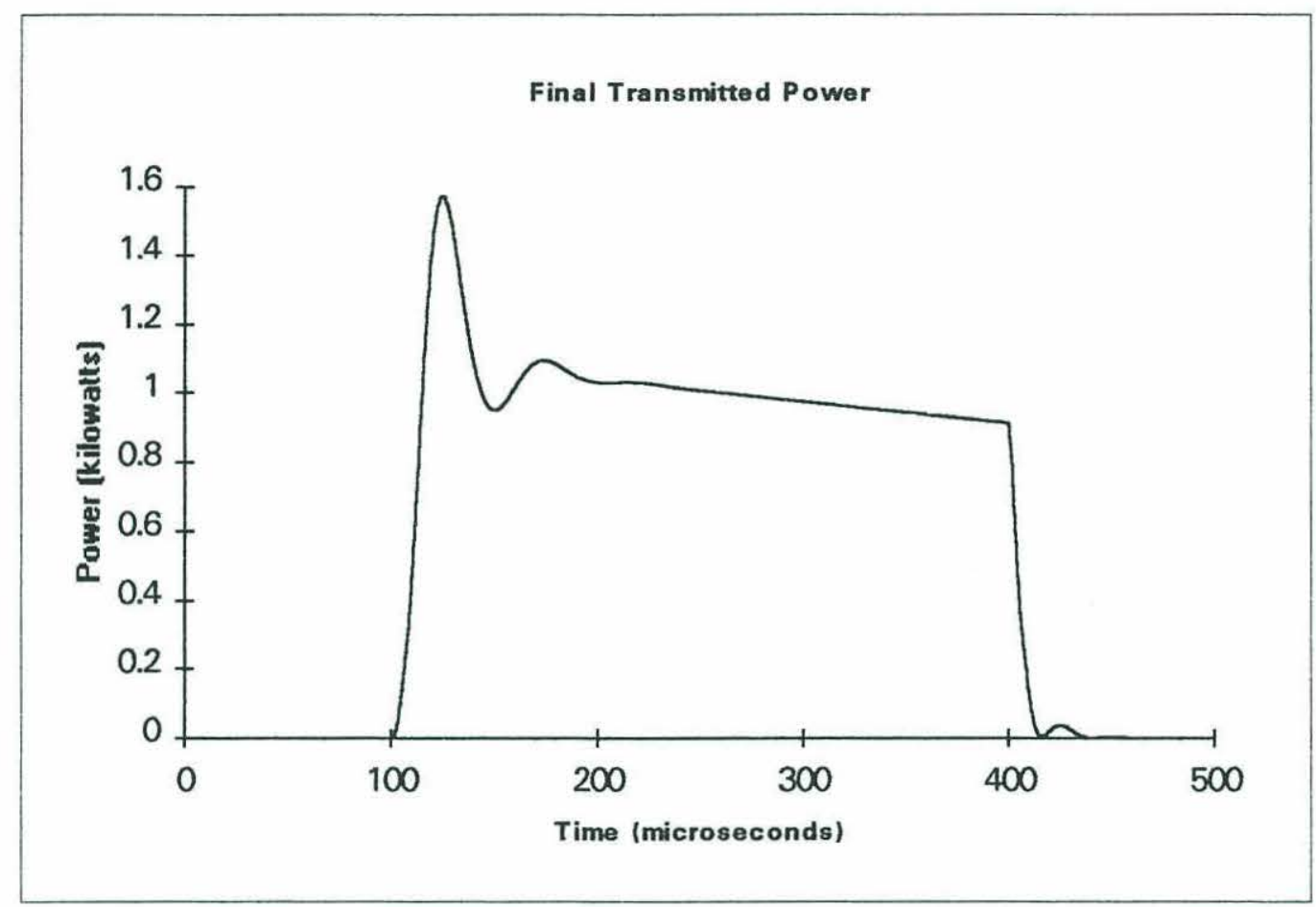

Fig 5.18 Peak power envelope of the final transmitted pulse.

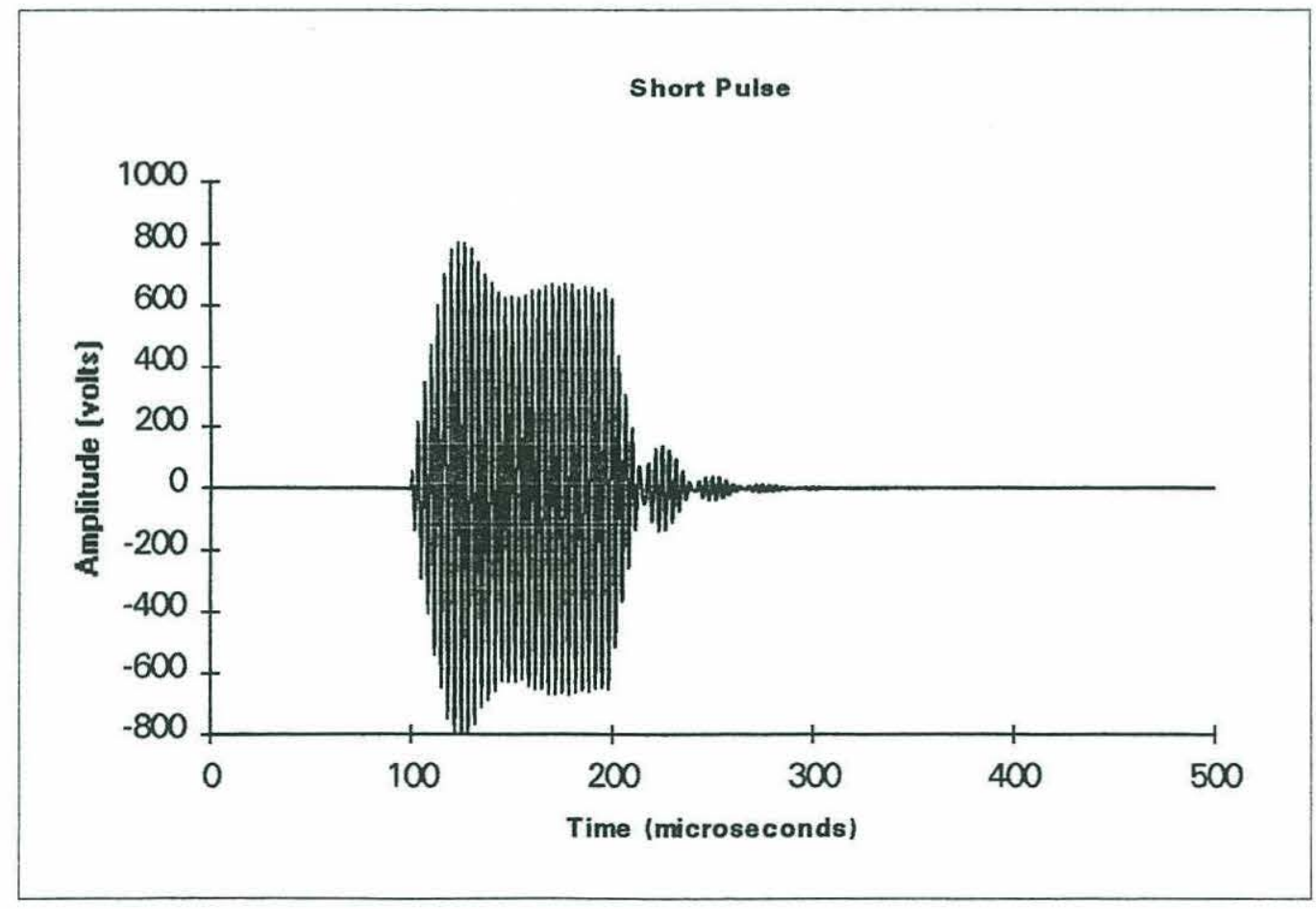

Fig 5.19 Transmitted pulse when $100 \mu \mathrm{sec}$ is selected. 


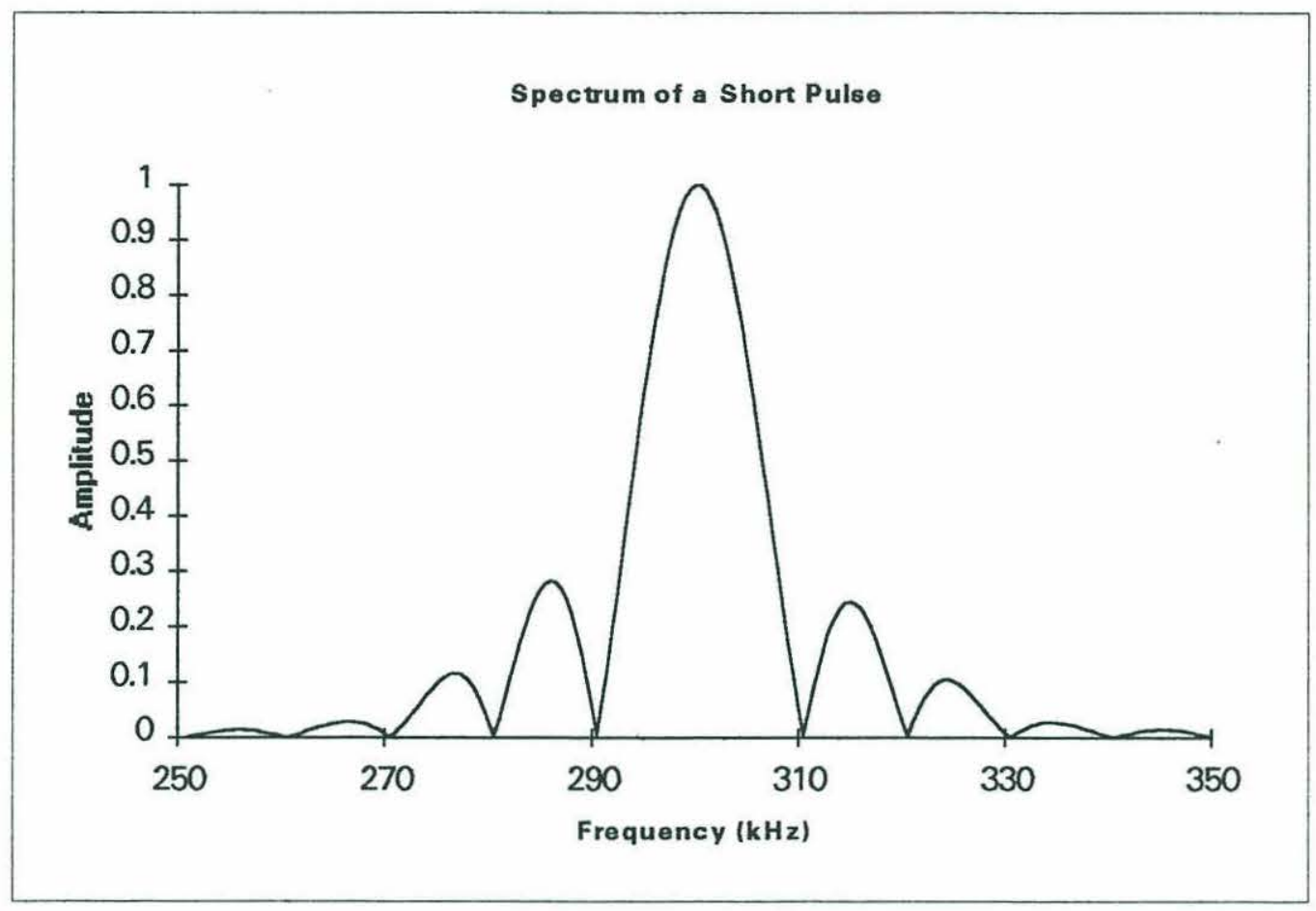

Fig 5.20 Normalized frequency response of the $100 \mu \mathrm{sec}$ pulse.

nominal amplitude to produce a 100-microvolt signal at the input to the first-stage amplifier.

\subsection{The Receiver Section}

Despite the remarkable advances in modern electronics, it is still not possible to reproduce the received signal without some corruption. In order to extract subtle characteristics of the return for environmental classification, it is necessary to understand what these corrupting effects are and to model them accurately.

The incoming return first accounters the transducer, which must be modeled somewhat differently than when used as a projector. Fig 5.21 illustrates the transducer equivalent circuit as it appears to the incoming signal. The equivalent voltage now 
appears in series with the radiation resistance, $R R$, the loss resistance, $R L$, the equivalent inductance, $\mathrm{L}$, and the capacitance, $\mathrm{C}$. The output is taken across the parallel combination of the shunt resistance, Ro, and the shunt capacitance, Co. The blocking components effectively form a voltage divider with the motional components. If no further modifications are made, the receiver will operate at point two of the impedance circle, as indicated in Fig 5.7. As indicated in Fig 5.21, the parallel capacitance and resistance across the input to the coupling transformer changes the operating frequency and the $\mathrm{Q}$ of the response [17].

The output of the equivalent circuit is given by:

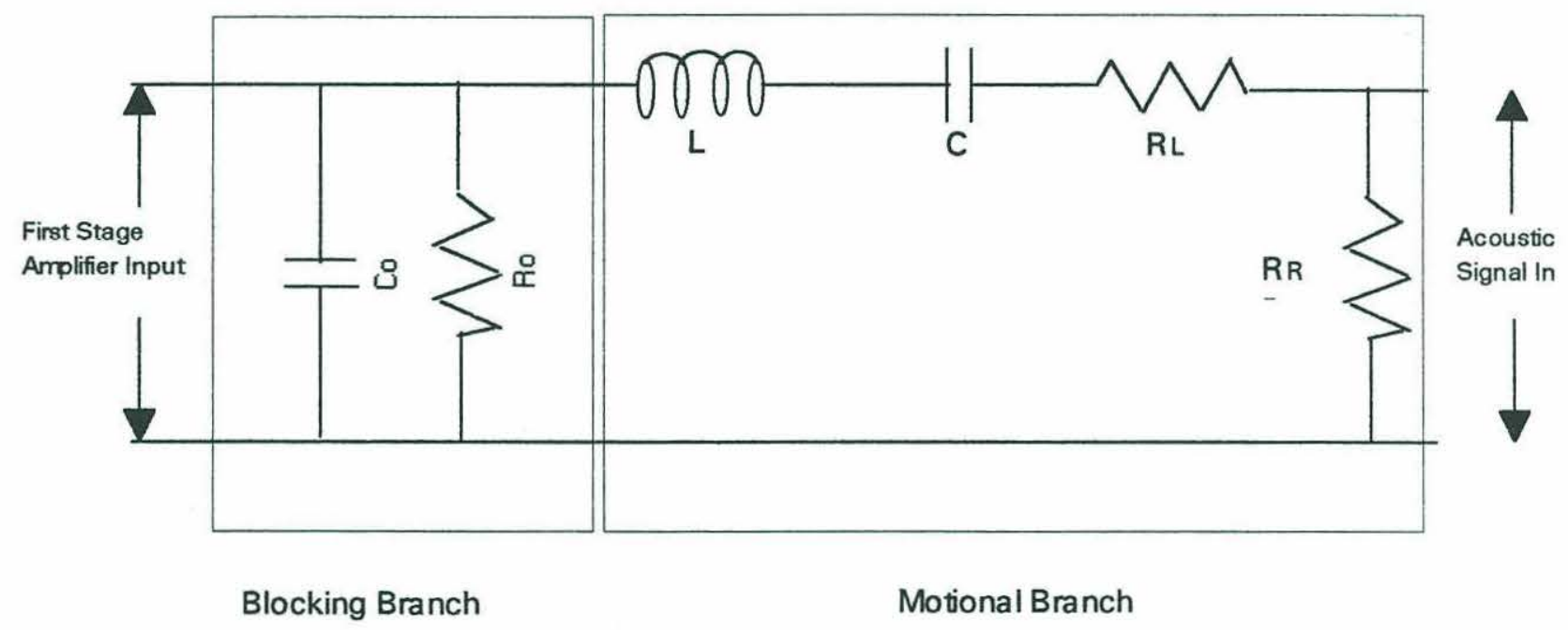

Fig 5.21 Transducer equivalent circuit as it appears to the incoming acoustic signal.

$$
\mathrm{Htr}_{\mathrm{tr}}(\omega)=\frac{\frac{1}{\frac{1}{\mathrm{R}_{1}}+j \omega \mathrm{C}_{1}}}{\frac{1}{\frac{1}{\mathrm{R}_{1}}+j \omega \mathrm{C}_{1}}+\mathrm{RL}-\frac{j}{\omega \mathrm{C}}+j \omega \mathrm{L}}
$$


where $R_{1}$ represents the equivalent resistance and $C_{1}$ the equivalent capacitance of the blocking branch. These will be a combination of the intrinsic shunt elements, Co and Ro, with discrete circuit components. Rearranging and substituting known values yields:

$$
H_{t r}(f)=\frac{\left(a_{4} f^{4}+a_{2} f^{2}\right)+j\left(b_{3} f^{3}+b_{11}\right)}{\left(c_{6} f^{6}+c_{4} f^{4}+c_{2} f^{2}+c 0\right)}
$$

Expressing this in polar form:

$$
\begin{gathered}
H_{t r(m a g)}(t)=\left(\frac{a 8 f^{8}+a b f^{6}+a 4 f^{4}+a 2 f^{2}}{c 12 f^{12}+c 10 f^{10}+c 8 f^{8}+c 6 f^{6}+c 4 f^{4}+c 2 f^{2}+c 0}\right)^{1 / 2} \\
H_{t r(\text { phase })(f)}=\tan ^{-1}\left(\frac{a 3 f^{3}+a l f}{c 4 f^{4}+c 2 f^{2}}\right)
\end{gathered}
$$

Appropriate coefficients are given by Table 5.3. The magnitude and phase responses are plotted in Figs 5.22 and 5.23. Fig 5.23 is the impulse response, htr, of the filter. The voltage signal at the transducer output, $V_{d}$ is:

$$
V_{d}(t)=\left(R_{R} B(0) P_{r}(t)\right)^{1 / 2} * h_{t r}(t)
$$

$\operatorname{Pr}$ here is the power of the returned pulse. $\mathrm{V}_{\mathrm{d}}$ is plotted in Fig 5.25 and its spectrum in Fig 5.26.

The model indicates that the transducer output is mixed with a noise input prior to injection into the first-stage amplifier. The noise actually consists of two parts: the correlated noise, or crosstalk, is a result of feedback from an adjacent channel, while 
the uncorrelated noise (labeled simply "noise" in the diagram) results from thermal and quantum effects. The noise input is placed before the first-stage amplifier for simplicity and because this is where practically all significant noise is generated.

\section{Equation 5.16 Equation 5.17a $\quad$ Equation 5.17b}

a0

a1

a2

a3

a4

a5

a6

a7

a8

b0

b1

b2

b3

c0

c1

c2

c3

c4

c5

c6

c7

c8

c9

c10

c11

c12
-

$3.6 \times 10^{-5}$

$1.2 \times 10^{-7}$

$-3.5 \times 10^{-9}$

$-$

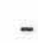

-

-

$6.0 \times 10^{-3}$

$-1.8 \times 10^{-7}$

1.0

$3.4 \times 10^{-3}$

-

$-6.8 \times 10^{-8}$

$3.4 \times 10^{-13}$
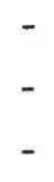

-

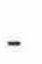

-
1.0

$6.9 \times 10$

$3.6 \times 10^{-4}$

$1.2 \times 10^{-5}$

$-3.5 \times 10^{-9}$

$-4.6 \times 10^{-10}$

$6.9 \times 10^{-15}$

$-4.6 \times 10^{-20}$

$1.1 \times 10^{-25}$
$6.0 \times 10^{-3}$

$-1.8 \times 10^{-7}$ 


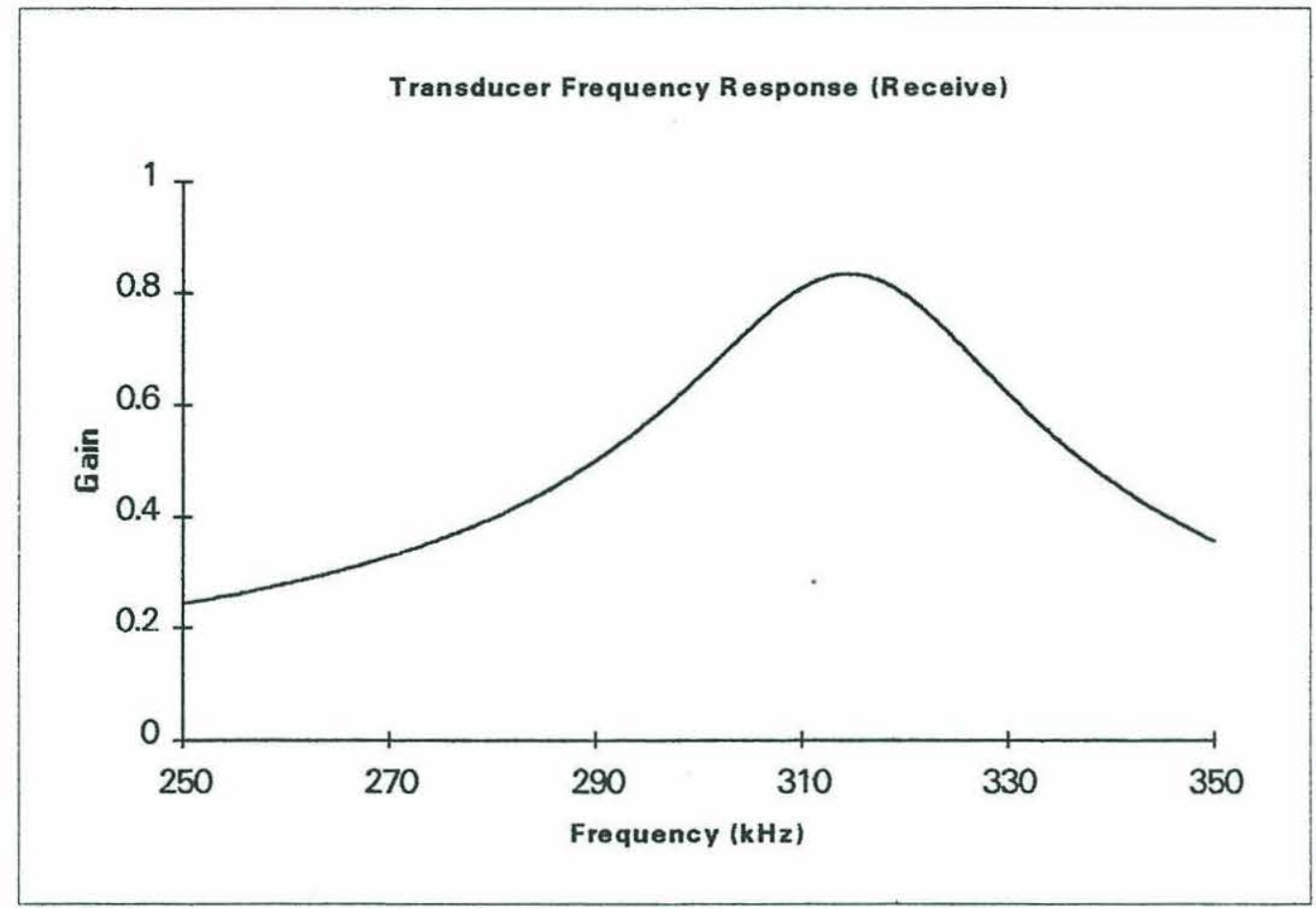

Fig 5.22 Frequency response of the transducer when used as a receiver.

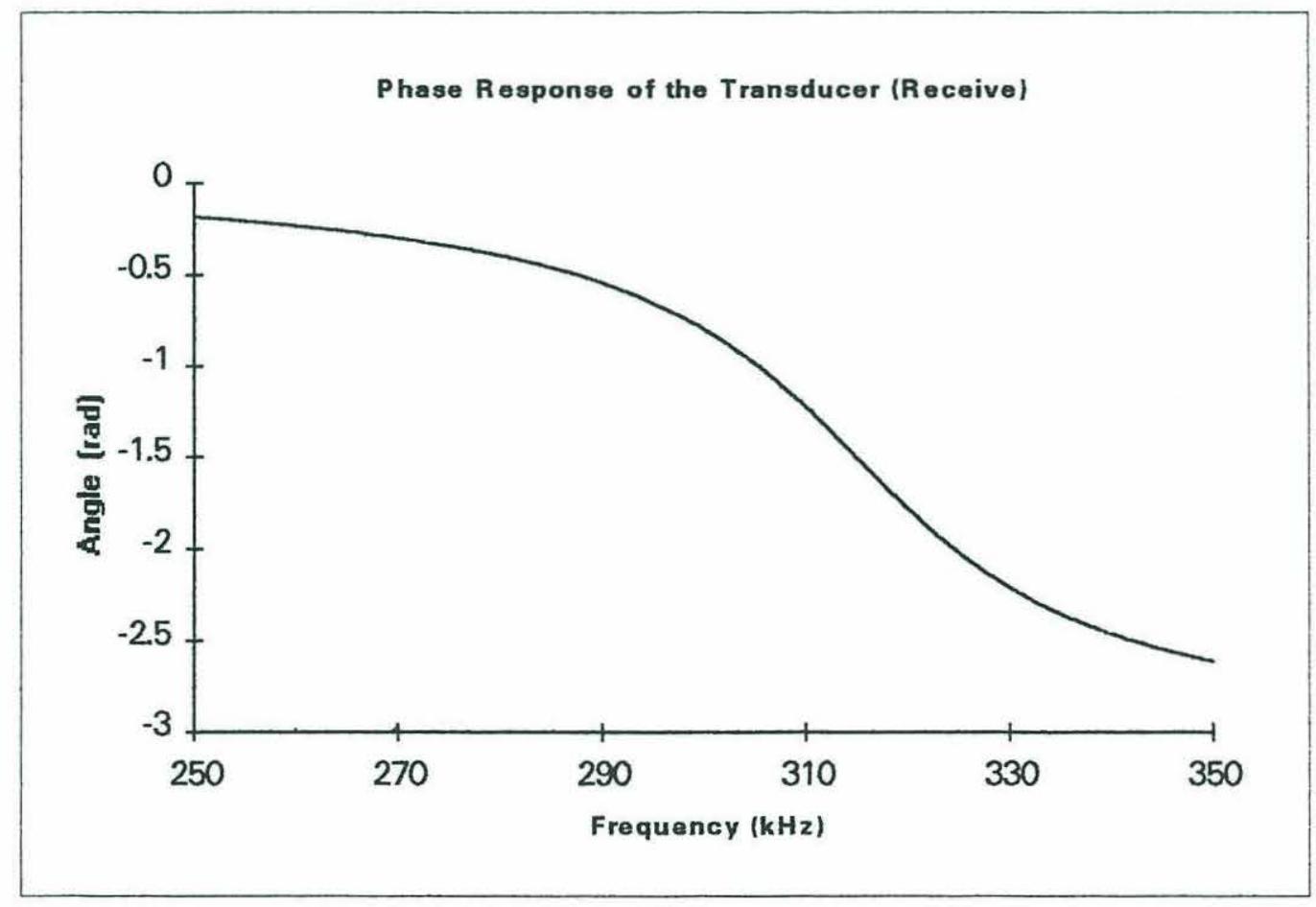

Fig 5.23 Phase response of the transducer when used as a receiver. 


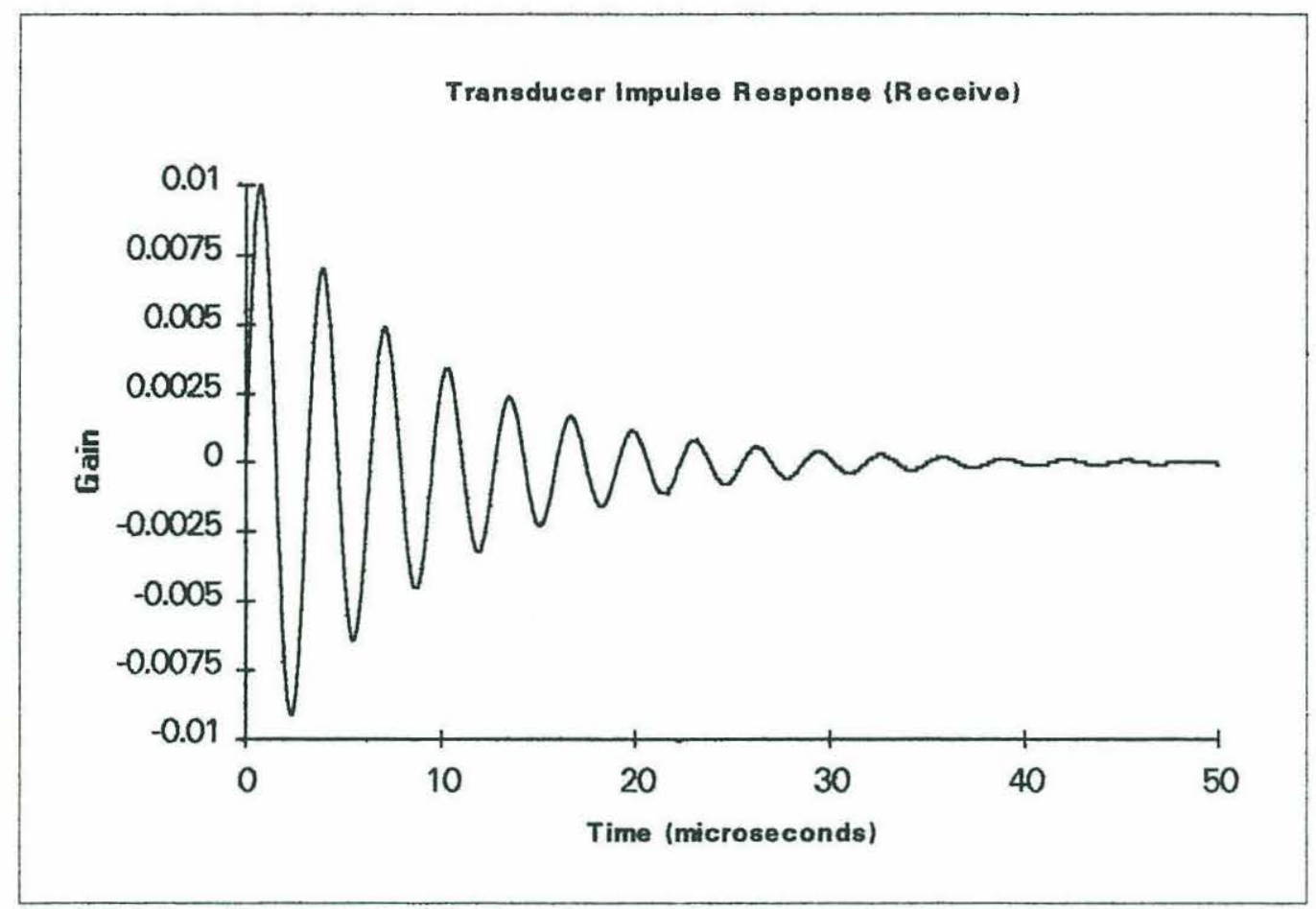

Fig 5.24 Impulse response of the transducer used as a receiver.

The uncorrelated noise results from several sources. "Johnson noise" is a temperature-dependent voltage fluctuation present across any resistor, regardless of whether or not an external input is present. The amplitude at any given time is distributed normally, while the frequency spectrum is flat, or "white". It is thus considered "Gaussian White Noise". Its amplitude is given by:

$$
\mathrm{V}_{\text {Doise }}(\mathrm{rms})=\sqrt{4 \mathrm{kTR} \beta}
$$

where $\mathrm{T}$ is the absolute temperature, $\mathrm{R}$ is the resistance in question, $\mathrm{k}$ is Boltzmann's constant, and $\beta$ is the bandwidth of measurement [14].

Since the flow of charge carriers is discrete rather than continuous, a resistor will 
also generate current noise due to statistical fluctuations in the flow. Known as "shot noise [11], this is also Gaussian and white. Its equation is:

$$
\mathrm{Inoise}=\sqrt{2 \mathrm{q} \mathrm{Idc}_{\mathrm{d}} \beta}
$$

where $\mathrm{q}$ is the electron charge and $\mathrm{Idc}_{\mathrm{dc}}$ is the a steady current through the device [14].

Another source of noise, known as "flicker noise", results from short-term fluctuations in the resistance itself. It is characterized by an inverse frequency dependence and is therefore called "pink noise" [14].

Since flicker noise is predominantly a low-frequency phenomenon, it can be safely ignored near our operating frequency of $300 \mathrm{kHz}$. Since the Johnson noise and the shot

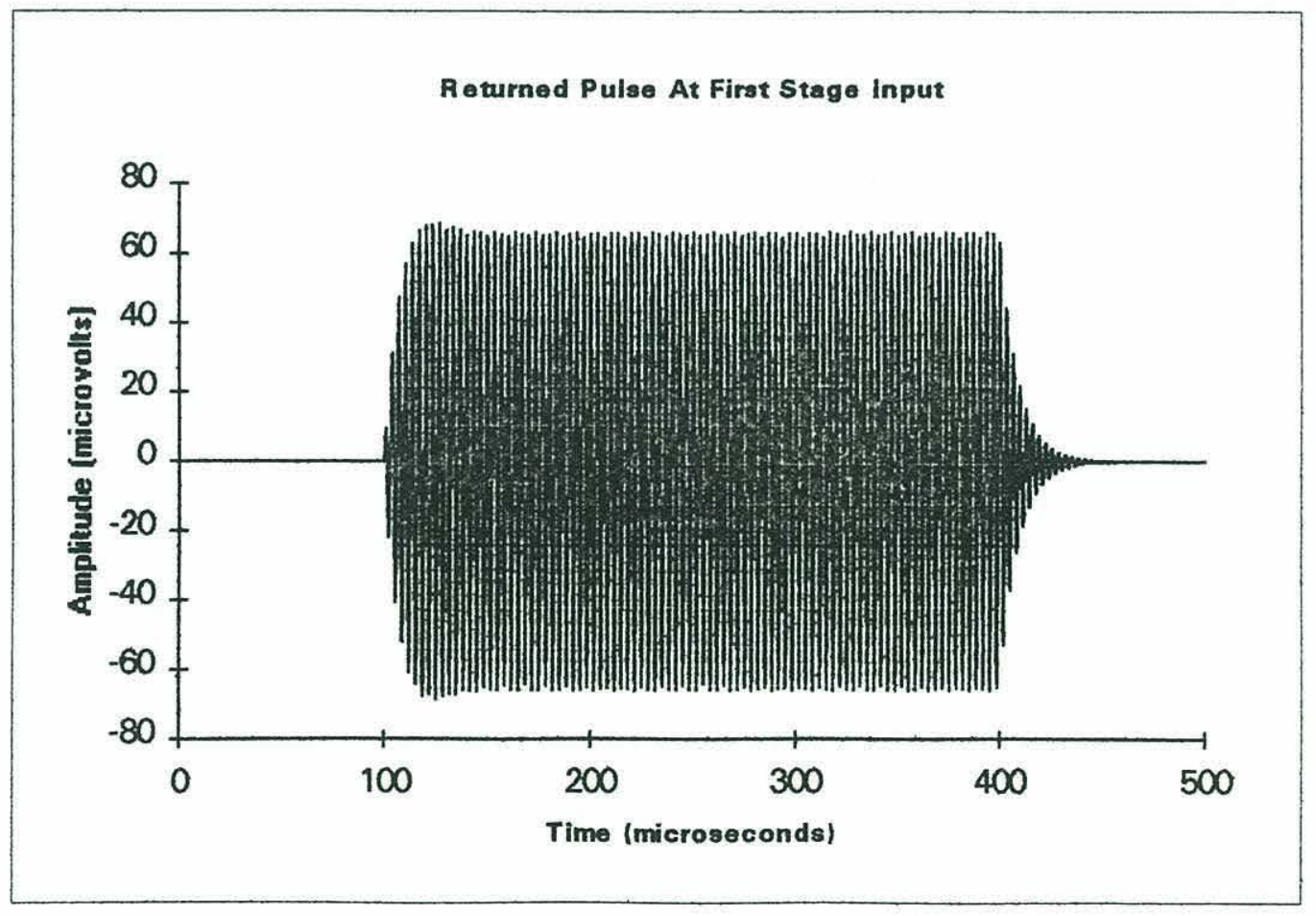

Fig 5.25 Returned pulse at the input to the first-stage amplifier. 


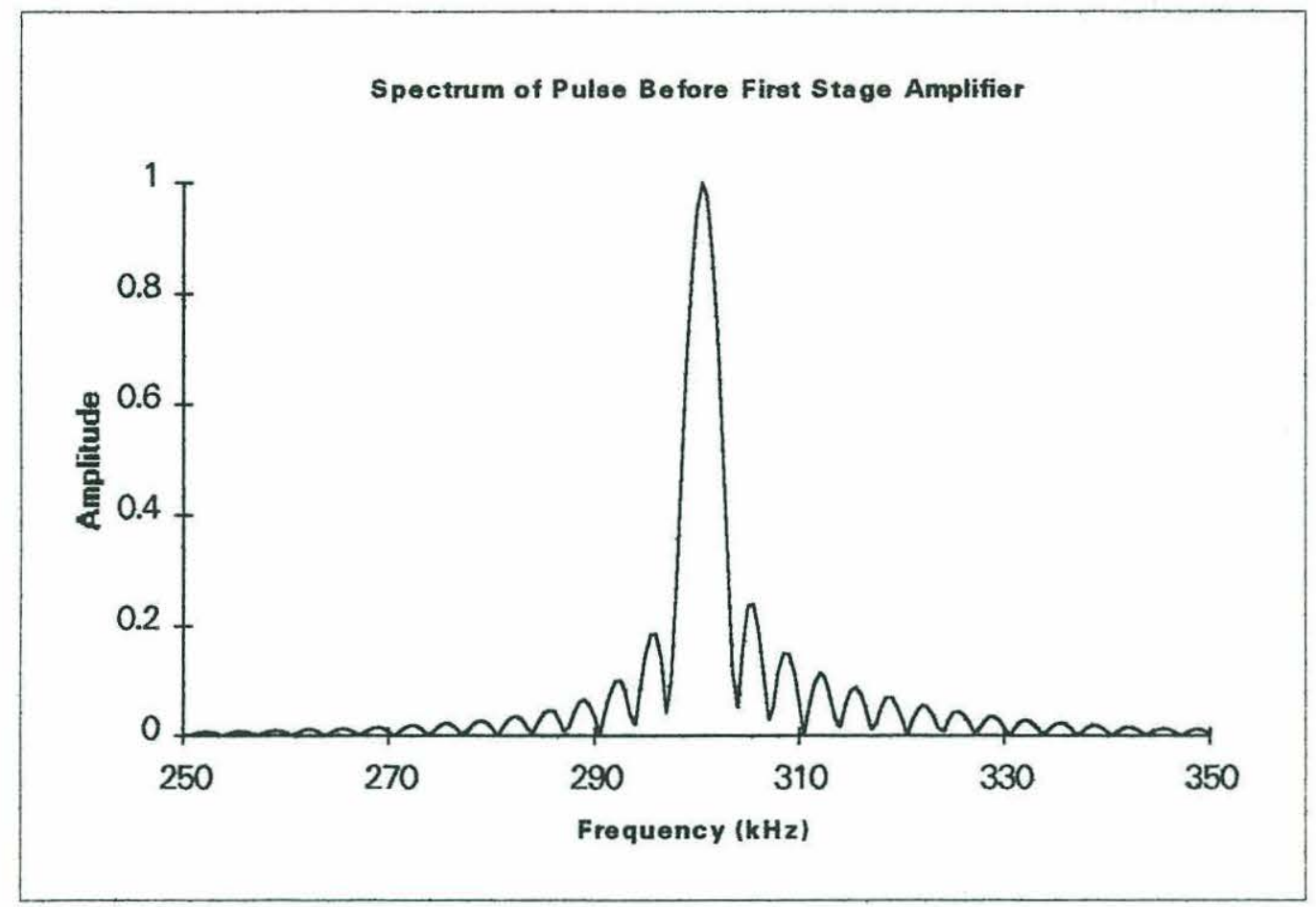

Fig 5.26 Spectrum of the pulse before the first-stage amplifier.

noise are both Gaussian and white, this is how the uncorrelated noise is modeled (Fig 5.27). The total uncorrelated noise will consist of a voltage noise source, en, in combination with the voltage drop across the source resistance, Rs, produced by the current noise, in [14]:

$$
\mathrm{V}_{\mathrm{n}}=\mathrm{en}_{\mathrm{n}}+\mathrm{i}_{\mathrm{n}} \mathrm{R}_{\mathrm{s}}
$$

Both en and in can be obtained from a few simple measurements. First, the amplifiers gain, $\mathrm{G}_{\mathrm{v}}$, is measured in the frequency band of operation. Next, the rms output voltage, $e_{s}$, is measured with the input shorted. The voltage noise is given by:

$$
\mathrm{e}_{\mathrm{n}}=\frac{\mathrm{e}_{\mathrm{s}}}{\mathrm{GvB}_{\mathrm{v}} \mathrm{B}^{1 / 2}} \mathrm{~V} / \mathrm{Hz}^{1 / 2}
$$


Next, a resistor $\mathrm{R}$ is placed across the amplifier input. This resistor is of arbitrary value, and is chosen to provide a significant amount of current noise. The new rms voltage output, er, can be used to determine in [14]:

$$
i_{n}=\frac{1}{R} \sqrt{\frac{e^{2}}{B G v^{2}}-\left(e^{2}+4 k T R\right)}
$$

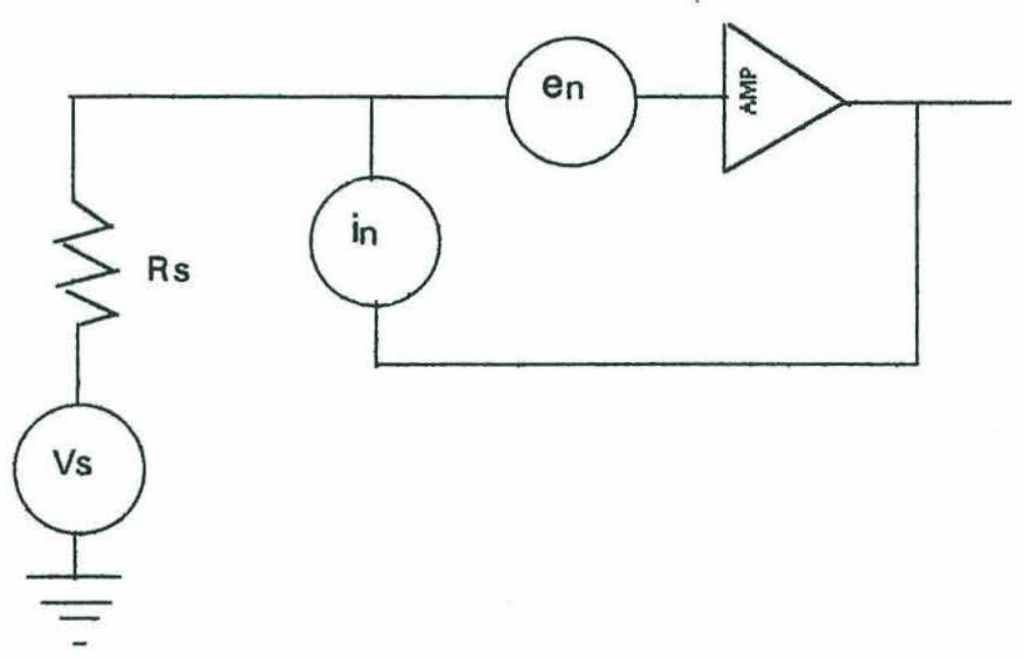

Fig 5.27 Model for determining amplifier noise.

These tests were conducted using the entire receiver network as the amplifier. A gain of $40 \mathrm{~dB}$ was selected. The results of the experiments are shown in Table 5.3. Fig 5.28 shows the uncorrelated noise input to the model. This noise was simulated using MATLAB's internal random number generator in its Gaussian mode.

Crosstalk can be directly measured simply by injecting a test signal into one receiver channel and measuring the output at adjacent channels. The only significant crosstalk found is between channels connected to the same transmitter and is caused by leakage through the diodes and transformer. Thus, there is crosstalk between channels $1 \mathrm{~A}$ and $2 \mathrm{~A}$ and channels $3 \mathrm{~A}$ and $4 \mathrm{~A}$. Its measured value is 0.005 percent. For 
illustration purposes, it is represented in the model as simply a copy of the returned signal delayed by to microseconds and attenuated by a factor $\mathrm{k}$.

$$
V_{x}(t)=k V_{d}\left(t-t_{d}\right)
$$

Fig 5.29 shows the signal as seen by the first-stage amplifier with noise and crosstalk included.

The first-stage amplifier itself can be modeled as a flat-gain element, since its rolloff begins far above any frequency of interest. Of course, there is a finite delay associated with the operational amplifier. This is combined with the delays of the other circuit elements and represented further downstream. The first-stage gain, $\mathrm{G}_{1}$, is 10.1, as demonstrated in Chapter 4. The signal after the first stage is given by:

$$
V_{\text {sigl } 1}(t)=G_{1}\left(V_{d}(t)+V_{n}(t)+V_{x}(t)\right)
$$

The pole-zero diagram of the bandpass filter is illustrated in Fig 5.30. This pattern can be translated into a frequency response:

$$
\operatorname{Hbp}(s)=\frac{1}{\left[s-\left(\omega 0 \pm j \sqrt{\omega c^{2}+\omega 0^{2}}\right)\right]\left[s-\left(\omega 0-\omega_{0}\right) \pm j \omega c \frac{\sqrt{3}}{2}\right]\left[s-\left(\omega 0+\omega_{c}\right) \pm j \omega \frac{\sqrt{3}}{2}\right]}
$$

The center frequency is $\omega_{0}$, and $\omega_{c}$ is half the filter bandwidth. This function can be conveniently plotted using the MATLAB function BUTTER. The resulting frequency response is shown in Fig 5.31, while the phase response is plotted in Fig 5.32. Fig 5.33 illustrates the impulse response, hbp. The bandpass output travels through a 


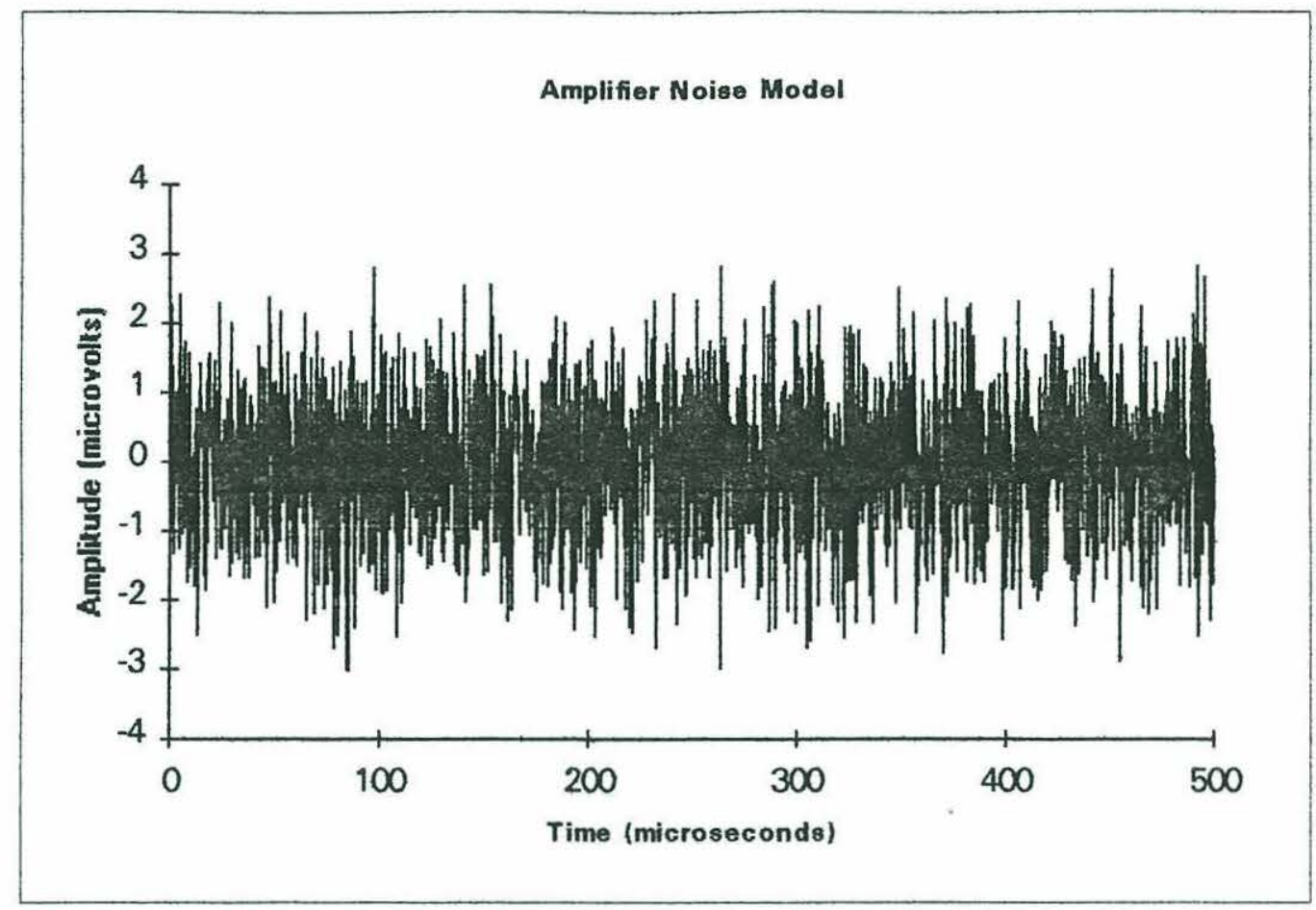

Fig 5.28 The amplifier noise is modeled as gaussian and white.

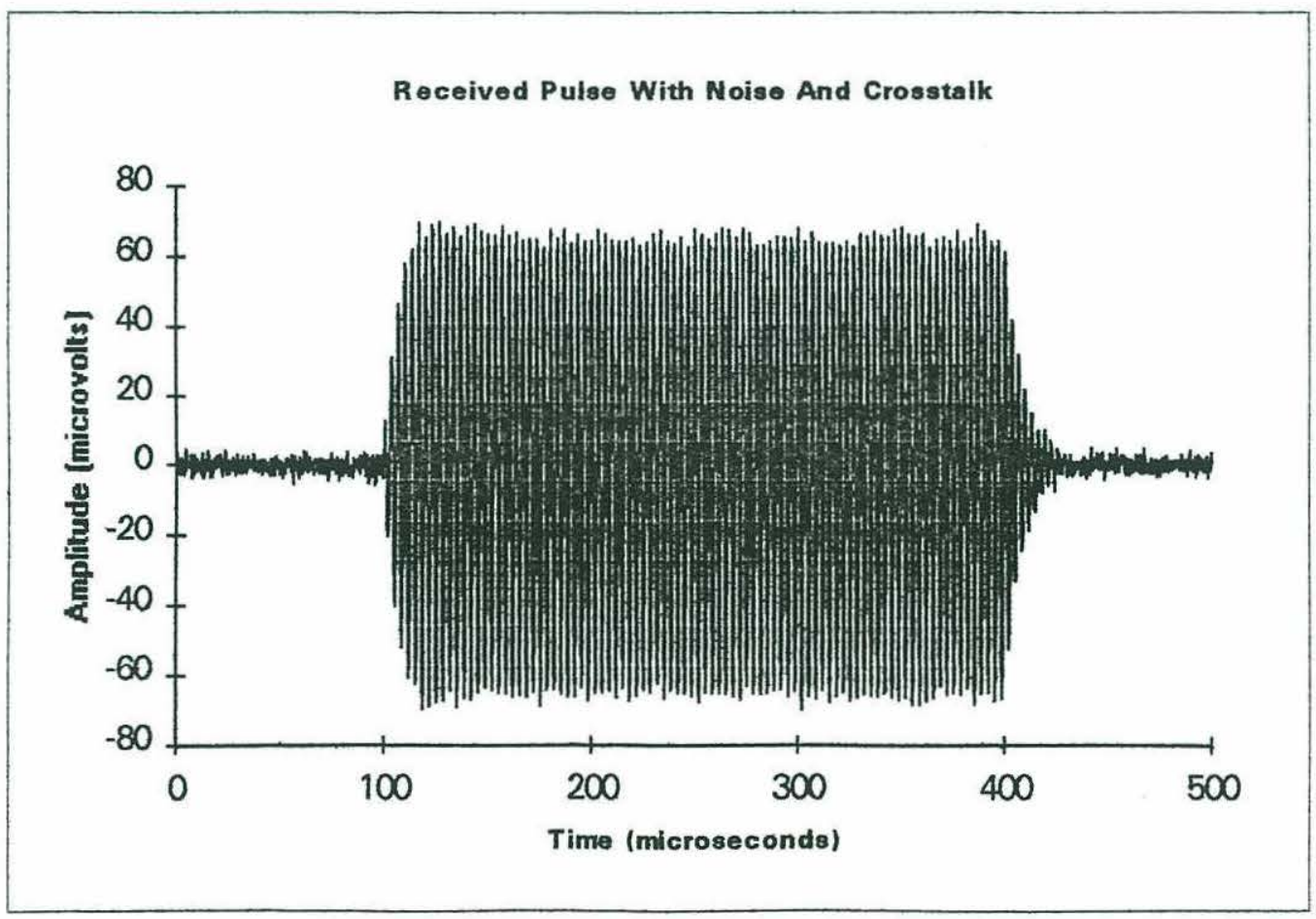

Fig 5.29 Pulse before the first stage amplifier with noise and crosstalk added. 
voltage divider and an inverting amplifier stage with a combined gain, $\mathrm{G} 2$, of -5 . The output from the second stage amplifier is given by:

$$
\mathrm{V}_{\mathrm{sig} 2}(\mathrm{t})=\mathrm{G}_{2}\left[\mathrm{~V}_{\mathrm{sig} 1}(\mathrm{t}) * \mathrm{hbp}_{\mathrm{p}}(\mathrm{t})\right] * h_{\text {delay1 }}(\mathrm{t})
$$

which is plotted in Fig 5.33 .

The delay element, delay 1 , is represented as an allpass filter with linear phase:

$$
\operatorname{Hdelay1}(\mathrm{mag})_{(\omega)}=1
$$

$$
H_{\text {delayl } 1 \text { phase })}(\omega)=\operatorname{td} \omega
$$

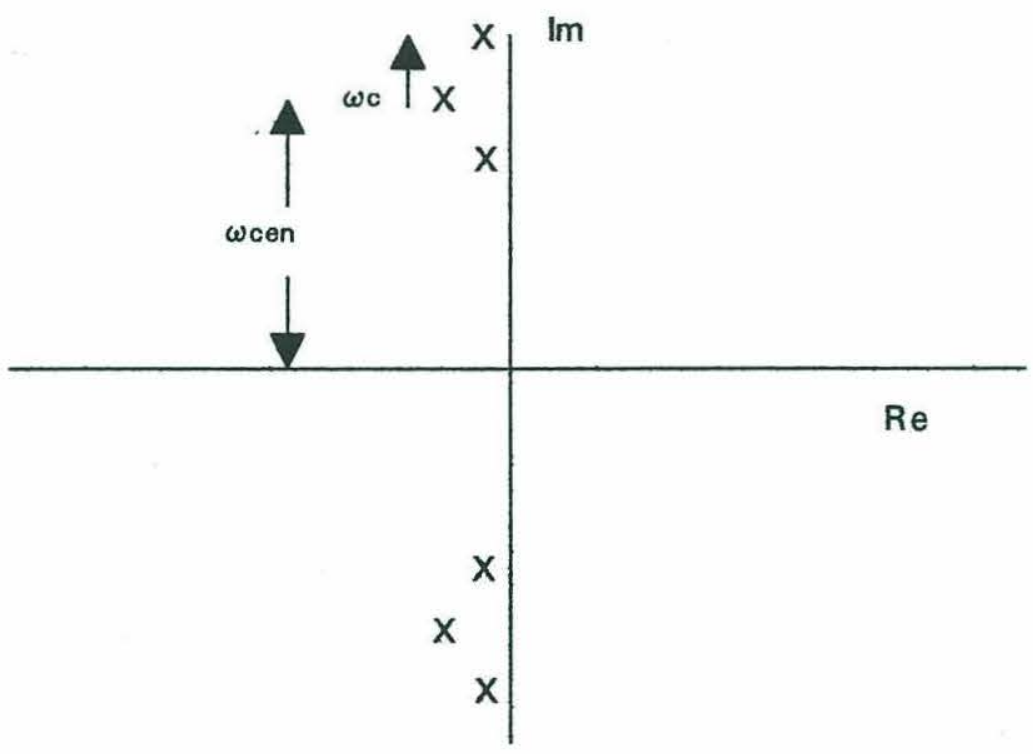

Fig 5.30 Poles of the butterworth bandpass filter. The poles form the negative half of a semicircle and are spaced 60 degrees apart. $\omega_{0}$ is the center frequency of the filter and $\omega_{c}$ is the cutoff frequency, which is half the bandwidth. 


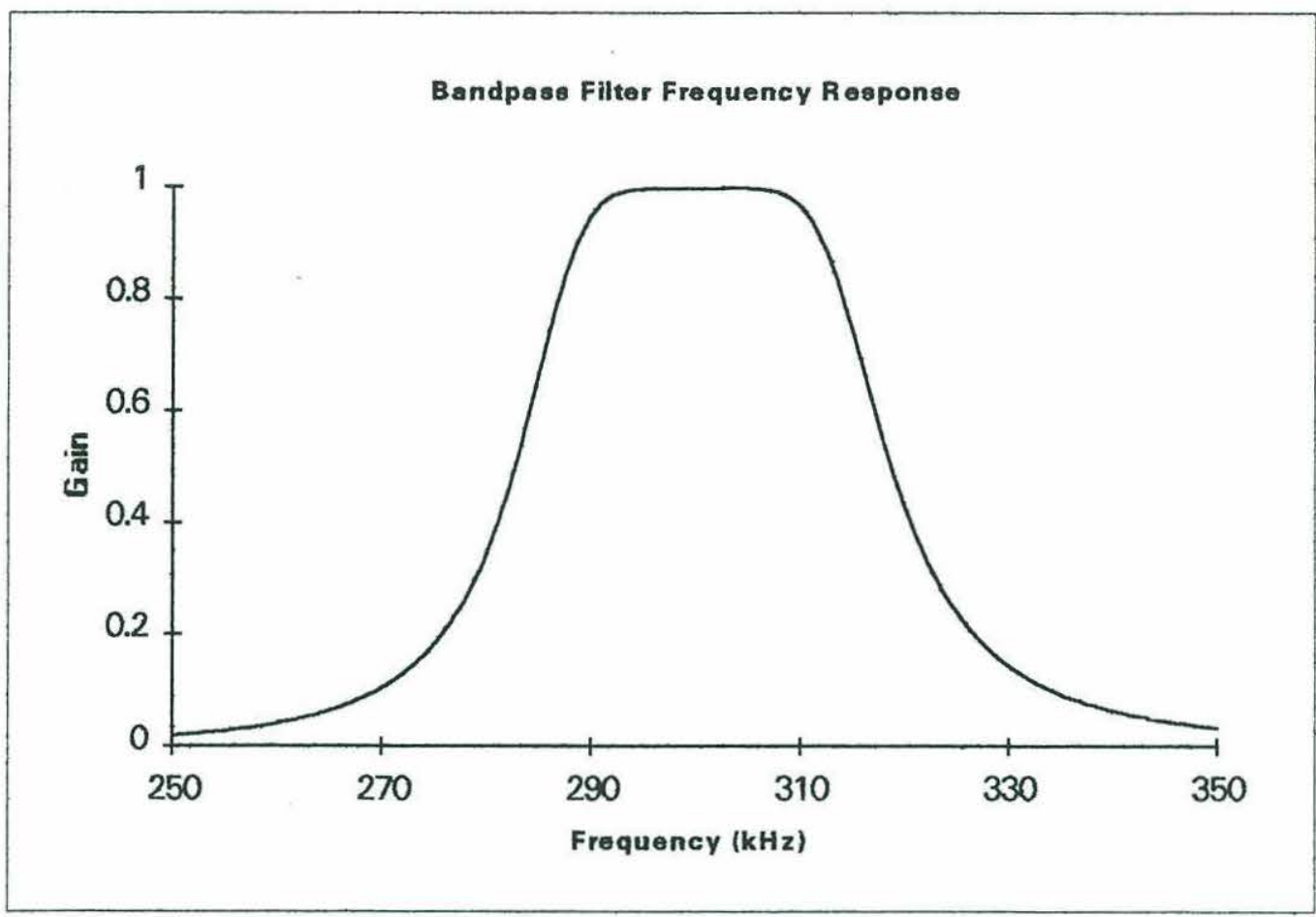

Fig 5.31 Butterworth bandpass filter frequency response.

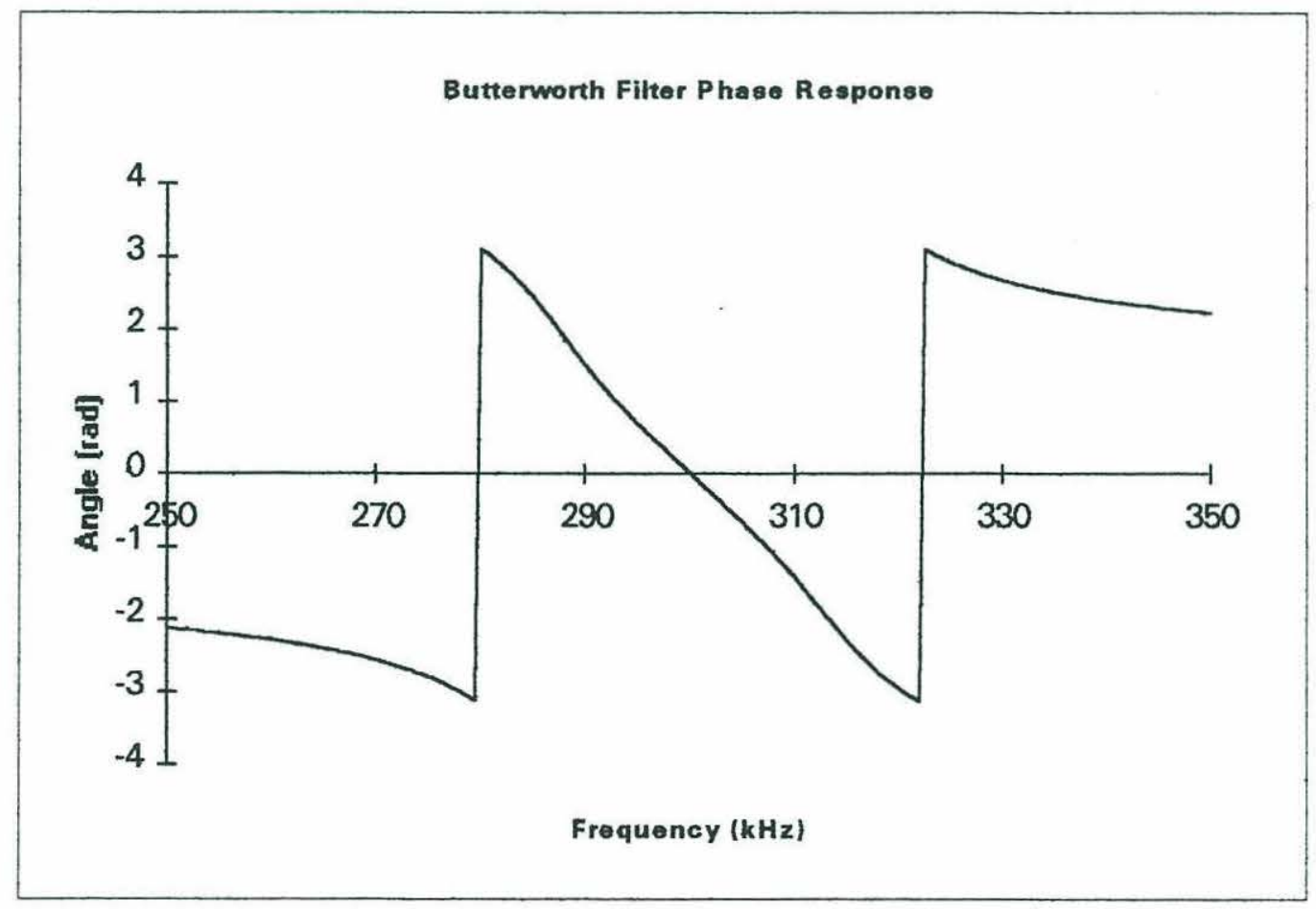

Fig 5.32 Butterworth filter phase response. 


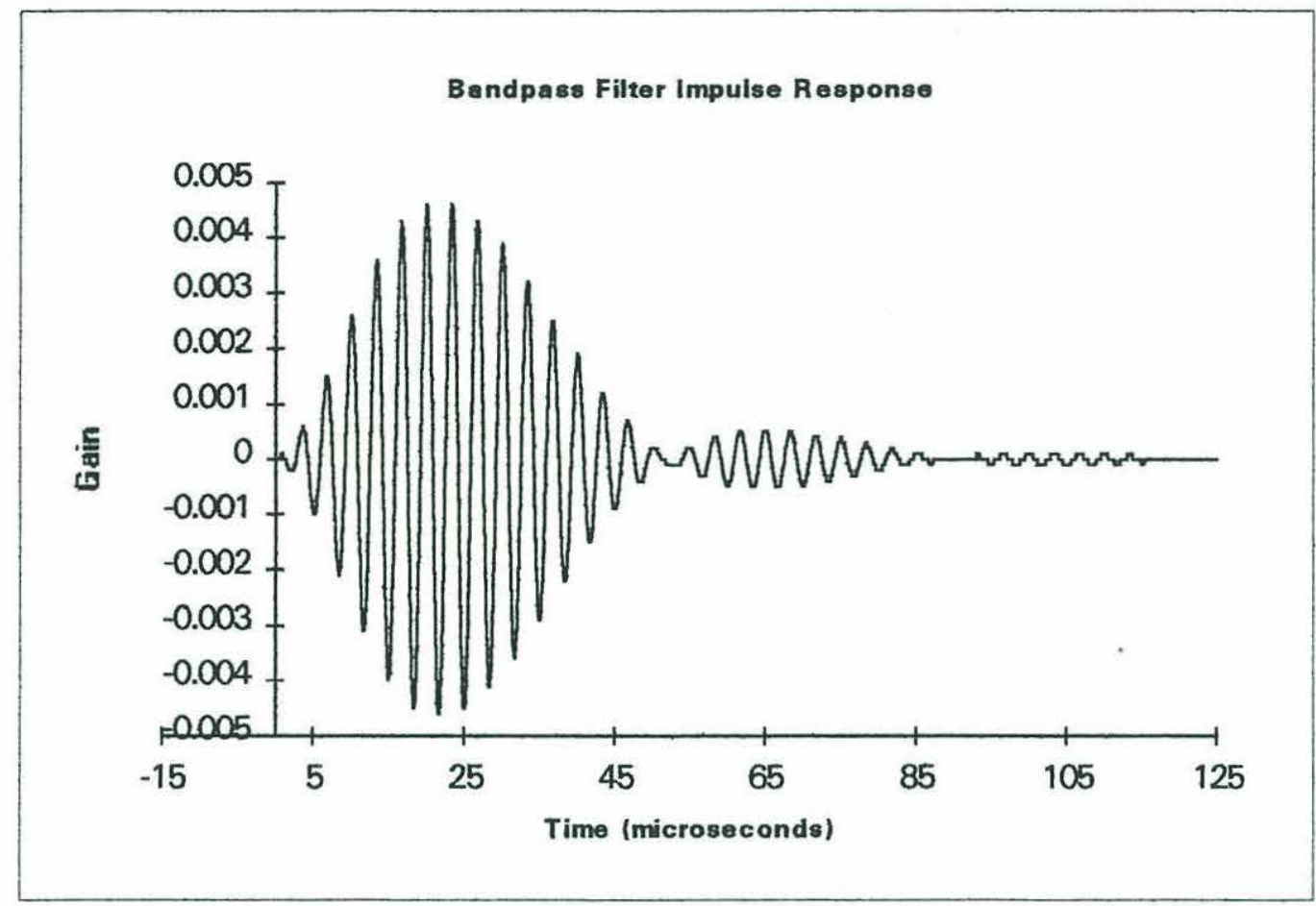

Fig 5.33 Impulse response of the bandpass filter.

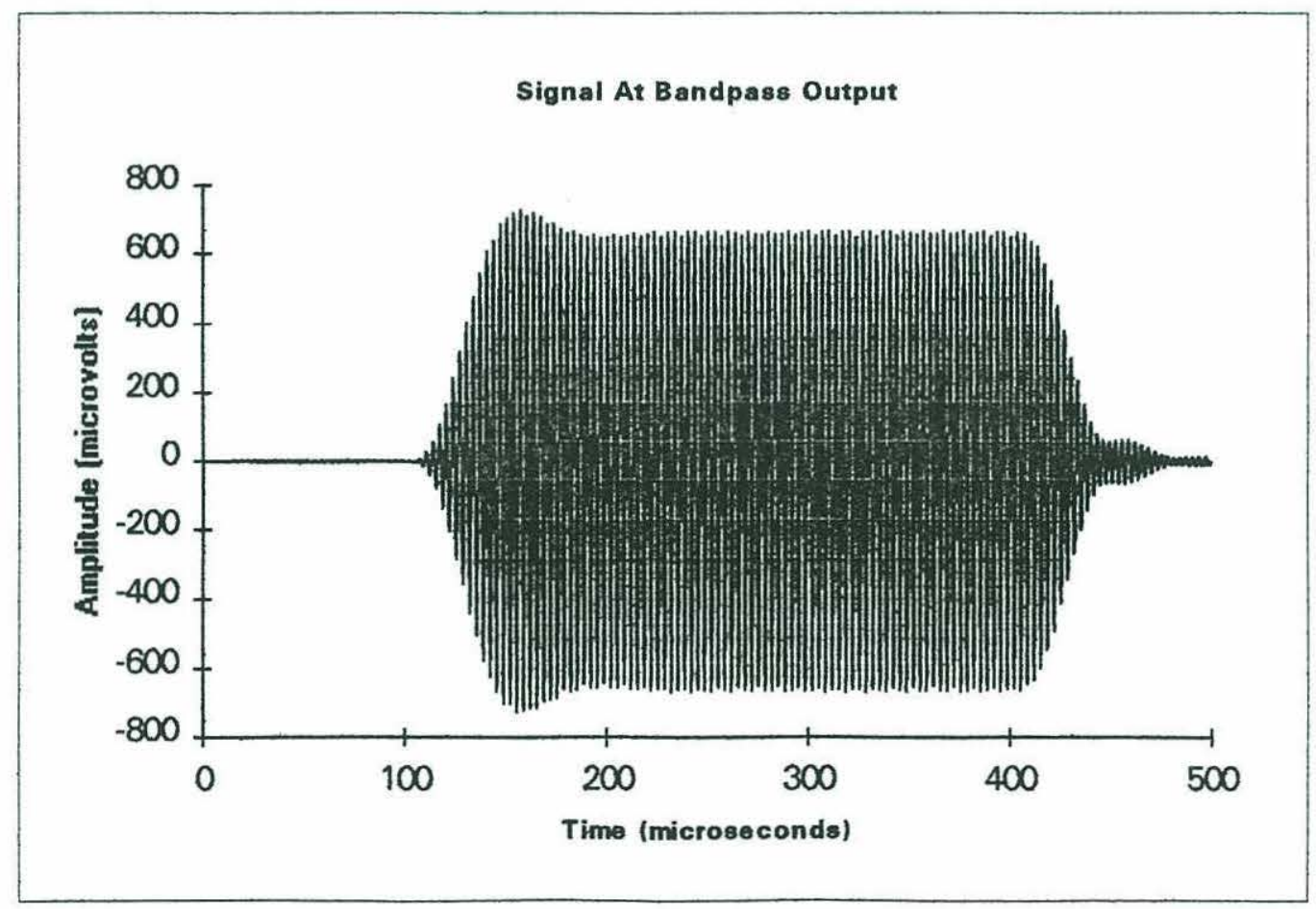

Fig 5.34 Received signal after bandpass filtering. 


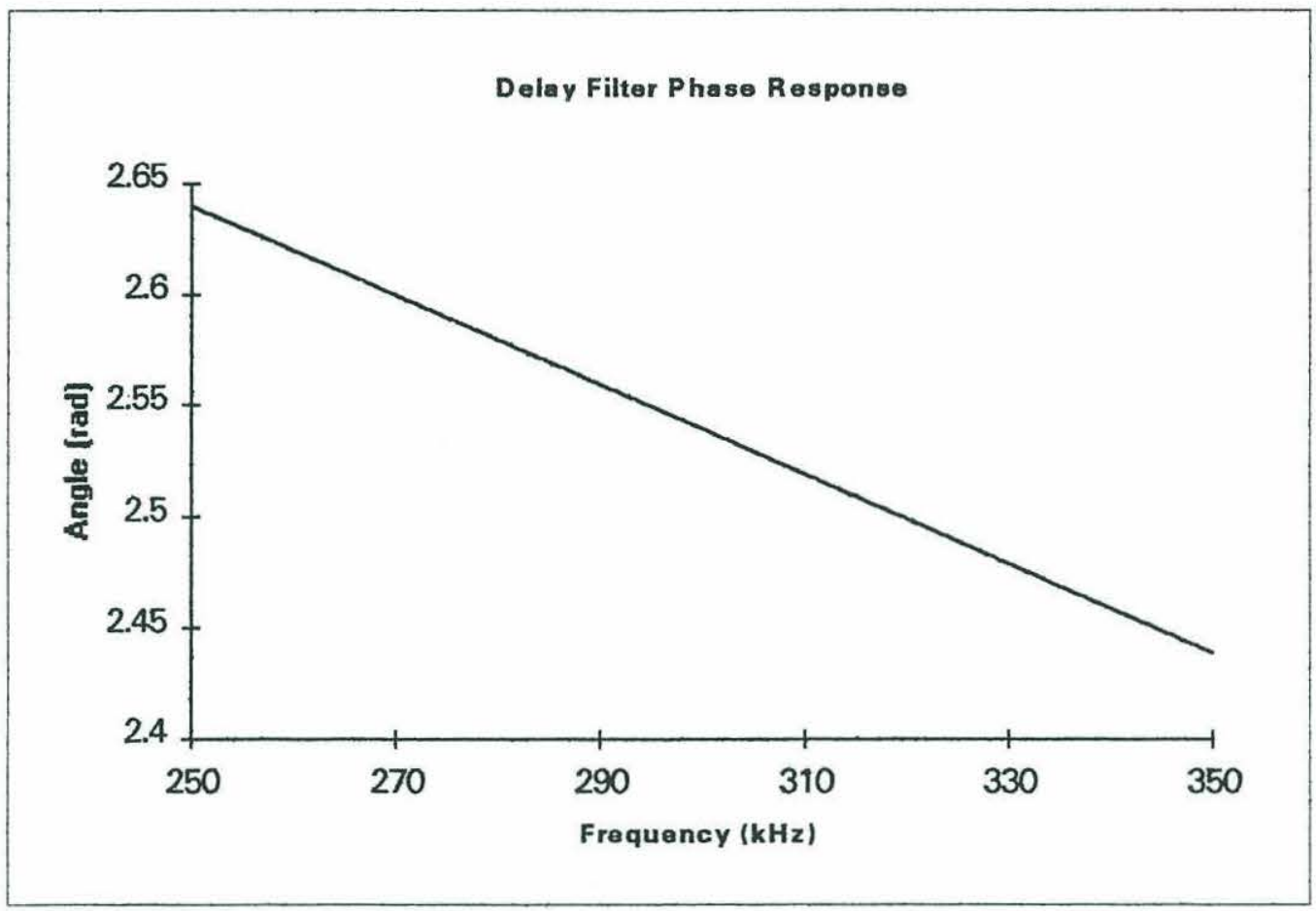

Fig 5.35 The delay elements are represented as allpass filters with linear phase.

as shown in Fig 5.35. The group delay of this filter is simply [18]:

$$
\mathrm{td}_{\mathrm{d}}=-(\mathrm{dH} \text { delayl}(\text { phase })(\omega) / \mathrm{d} \omega)
$$

The rest of the receiver circuitry consists of a network of operational amplifiers and phase compensating filters, as described in the previous chapter. The op-amps continue to be modeled as simple gain elements with a finite delay in each path. The compensating networks are represented by the equivalent circuit in Fig 5.36. Capacitor $\mathrm{C} 2$, in parallel with resistor $\mathrm{R} 2$, forms a voltage divider with resistor $\mathrm{R} 1$. This results in the following frequency response: 


$$
\mathrm{H}_{\mathrm{comp}}(\omega)=\frac{\frac{1}{j \omega \mathrm{C}+\frac{1}{\mathrm{R} 2}}}{\mathrm{R} 1+\frac{1}{j \omega \mathrm{C}+\frac{1}{\mathrm{R} 2}}}
$$

Recasting this in polar form yields:

$$
\begin{gathered}
H_{\text {comp(mag) }}(f)=\frac{\sqrt{\left(\mathrm{R} 1 \mathrm{R} 2+\mathrm{R} 2^{2}\right)^{2}+\left(2 \pi \mathrm{fCR} 1 \mathrm{R} 2^{2}\right)^{2}}}{(2 \pi \mathrm{fCR} 1 \mathrm{R} 2)^{2}+(\mathrm{R} 1+\mathrm{R} 2)^{2}} \\
H_{\text {comp(phase) }(\mathrm{f})}=\tan ^{-1}\left(\frac{-2 \pi \mathrm{fCR} 1 \mathrm{R} 2^{2}}{\mathrm{R} 1 \mathrm{R} 2+\mathrm{R} 2^{2}}\right)
\end{gathered}
$$

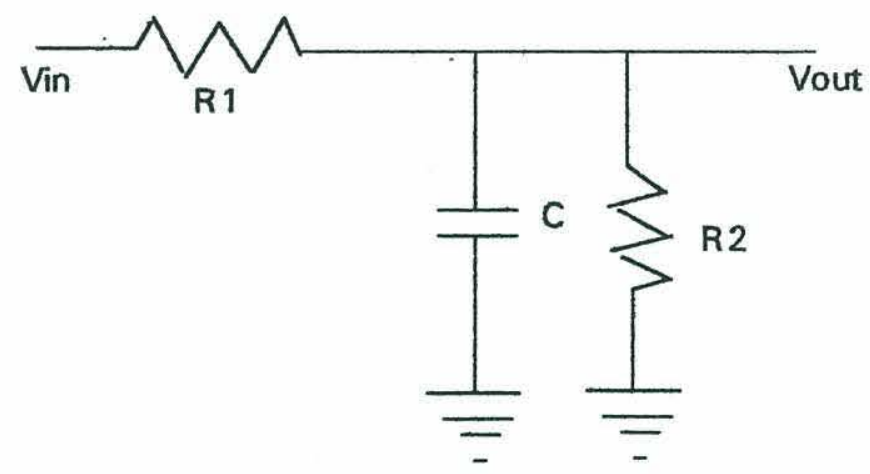

Fig 5.36 Equivalent circuit of the compensating filters. R2 represents the input resistance of the summing amplifier.

\begin{tabular}{|cccc|}
\hline & R1 & R2 & C \\
Comp 1 & 2000 ohms & 2000 ohms & $270 \mathrm{pF}$ \\
Comp 2 & 2000 ohms & 2000 ohms & $130 \mathrm{pF}$ \\
\hline
\end{tabular}

Table 5:4 Component values corresponding to Fig 5.34. 
Figs 5.37 and 5.38 illustrate the response for the $0 \mathrm{~dB}$ branch. Fig 5.39 illustrates the impulse response.

The final signal delivered to the sample and hold amplifier is given by:

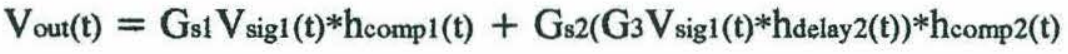

$$
\begin{aligned}
& +\mathrm{G}_{3} \mathrm{G}_{4} \mathrm{~V}_{\text {sig1 }}(\mathrm{t}) * \mathrm{~h}_{\text {delay3 }}(\mathrm{t})
\end{aligned}
$$

The meaning of the symbols in equation 5.35 is illustrated by Fig 5.40. Figs 5.41 and 5.42 show time and frequency plots of the final signal, respectively. Figs 5.43 and 5.44 illustrate the final result when the model transmitted pulse is used for an input signal.

A final problem concerns the sampling characteristics of the sample and hold amplifiers. Any real device will have some finite delay, known as aperture time, between the hold command and the actual freezing of the input signal [14]. There will also be a finite voltage droop rate due to current leakage in the holding capacitor. Although these errors are very small (on the order of $0.05 \%$ ) in comparison with signal amplitude, they can become significant when comparing the phase difference between two channels. This is especially true near peaks in the sinusoid, where the signal is changing very slowly. Although these errors can in theory be eliminated by proper adjustment of the amplifier offset input, they will not completely disappear in practice due to change in component values over time and temperature effects.

One way to determine the sample and hold errors is to average the signal amplitude 


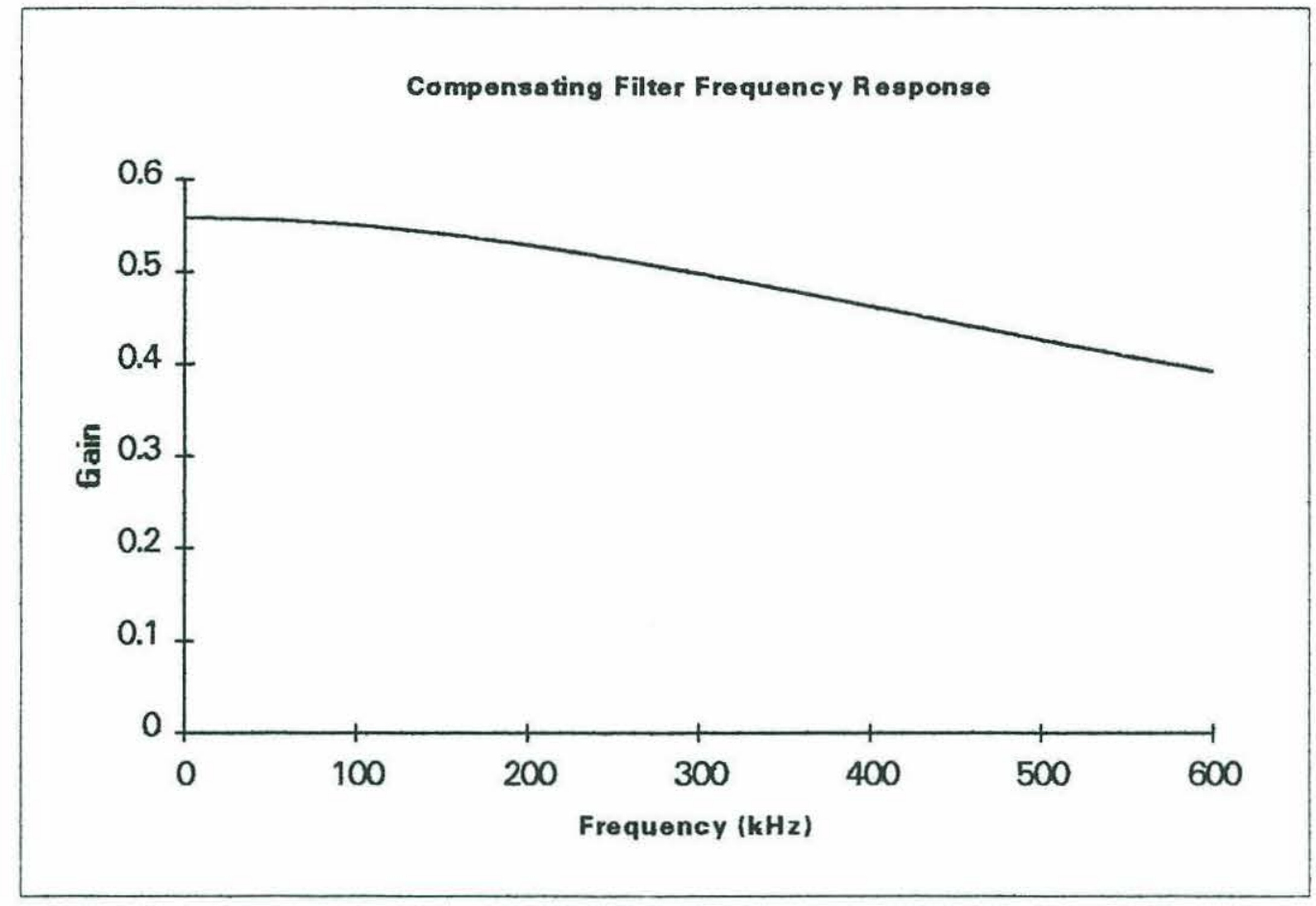

Fig 5.37 Frequency response of the $0 \mathrm{~dB}$ compensating filter.

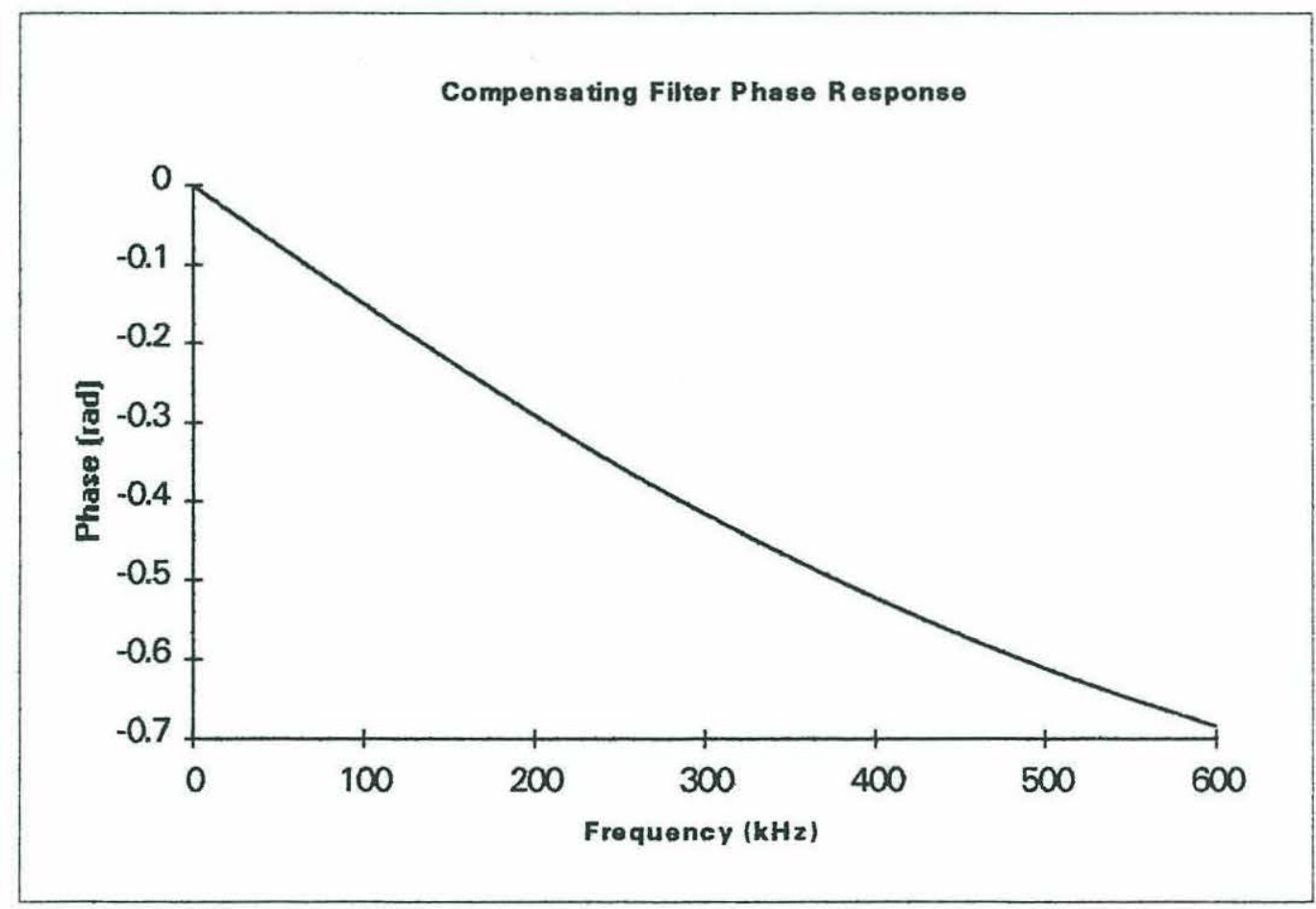

Fig 5.38 The nearly linear phase response of the compensating filter provides a time delay. 


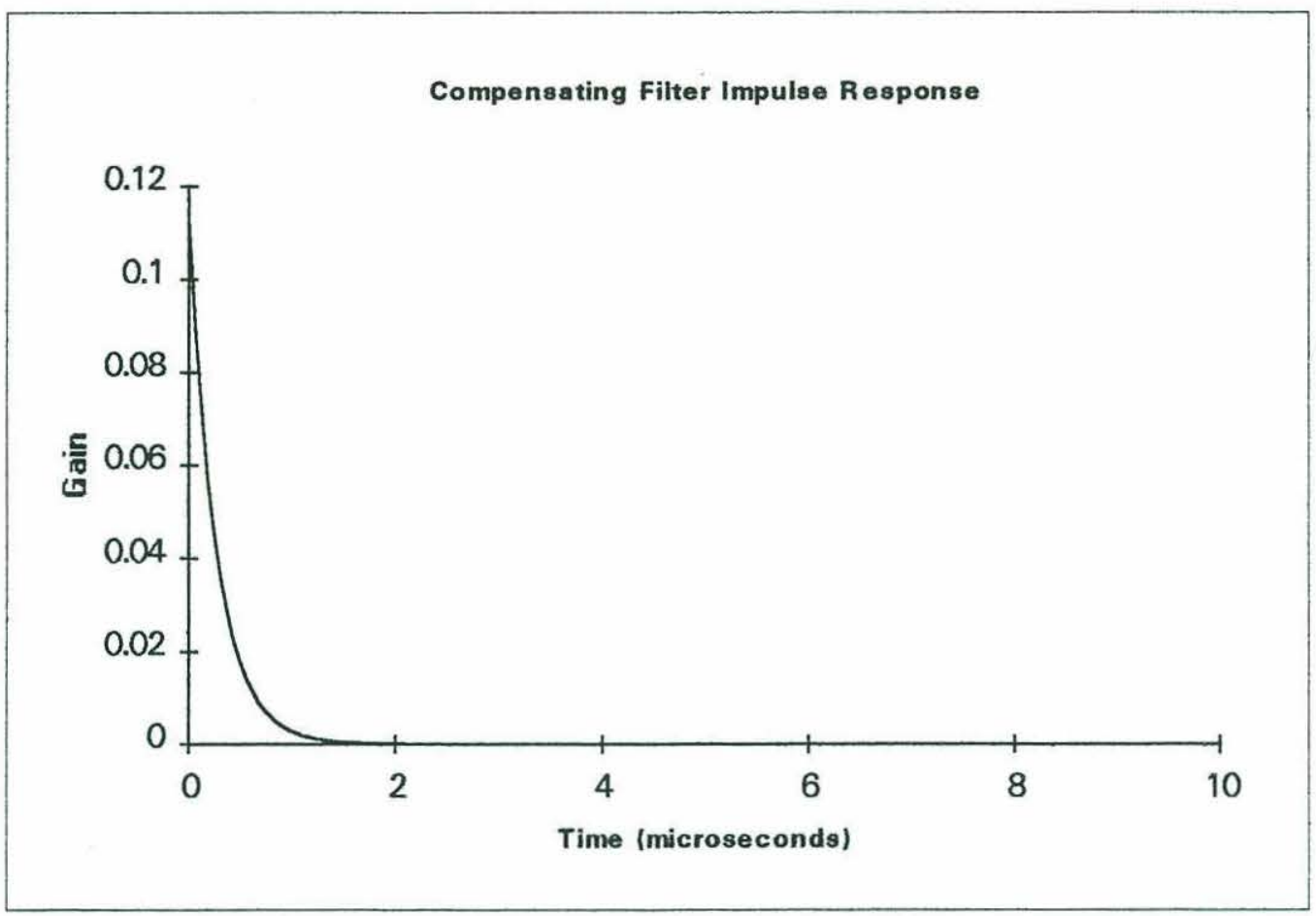

Fig 5.39 Compensating filter impulse response.

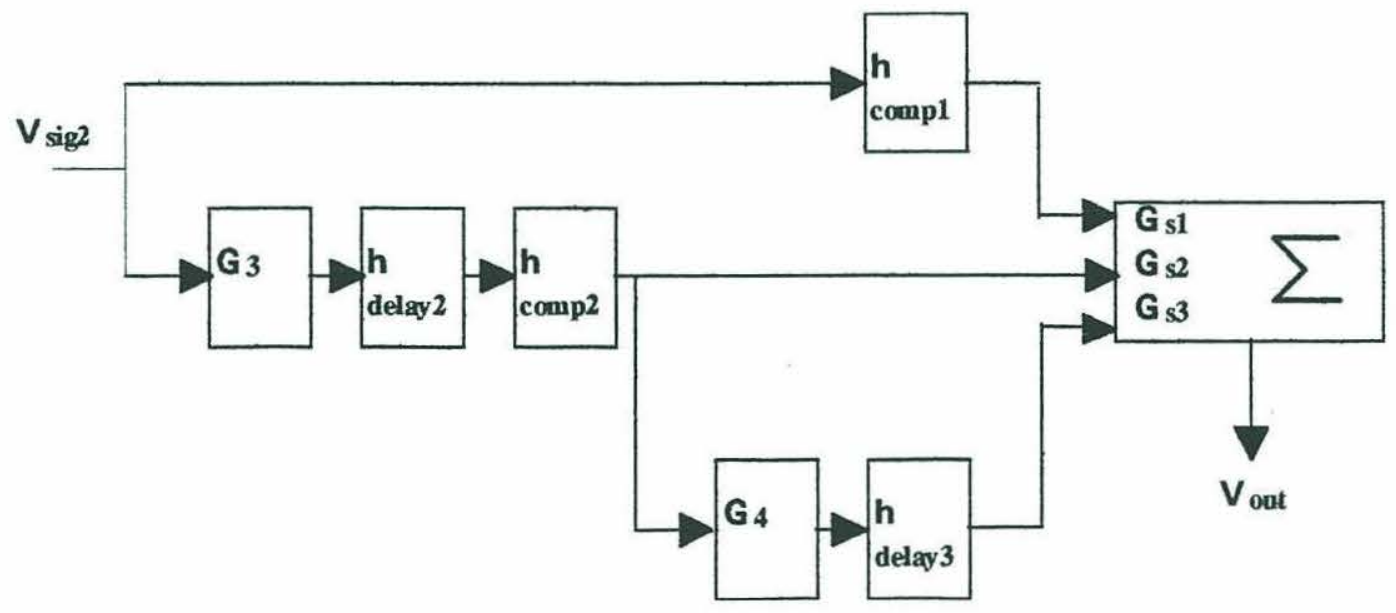

Fig 5.40 Schematic representation of the amplifier section of the receiver, illustrating the symbols used in equation 5.35 . 


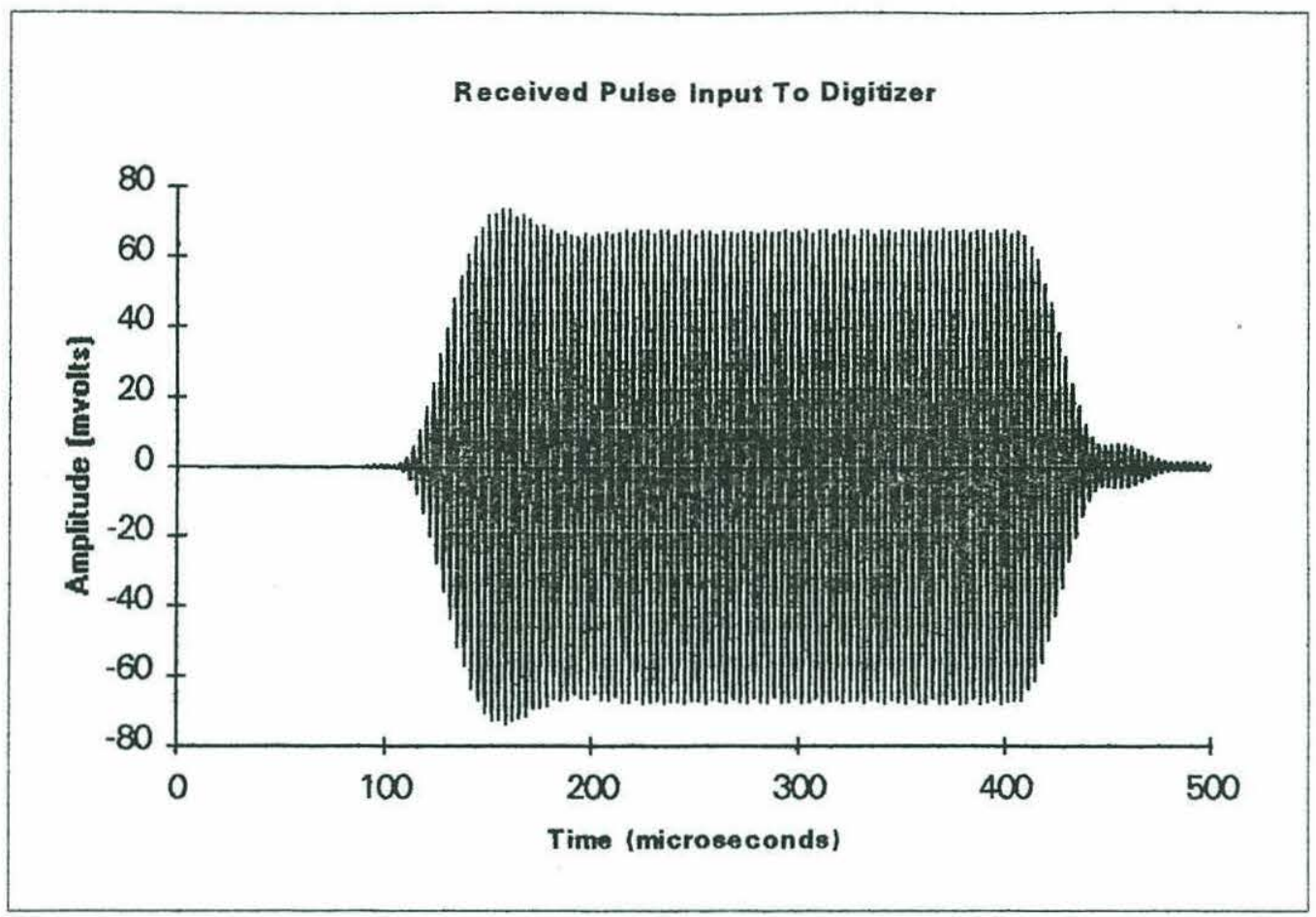

Fig 5.41 Final signal before sampling.

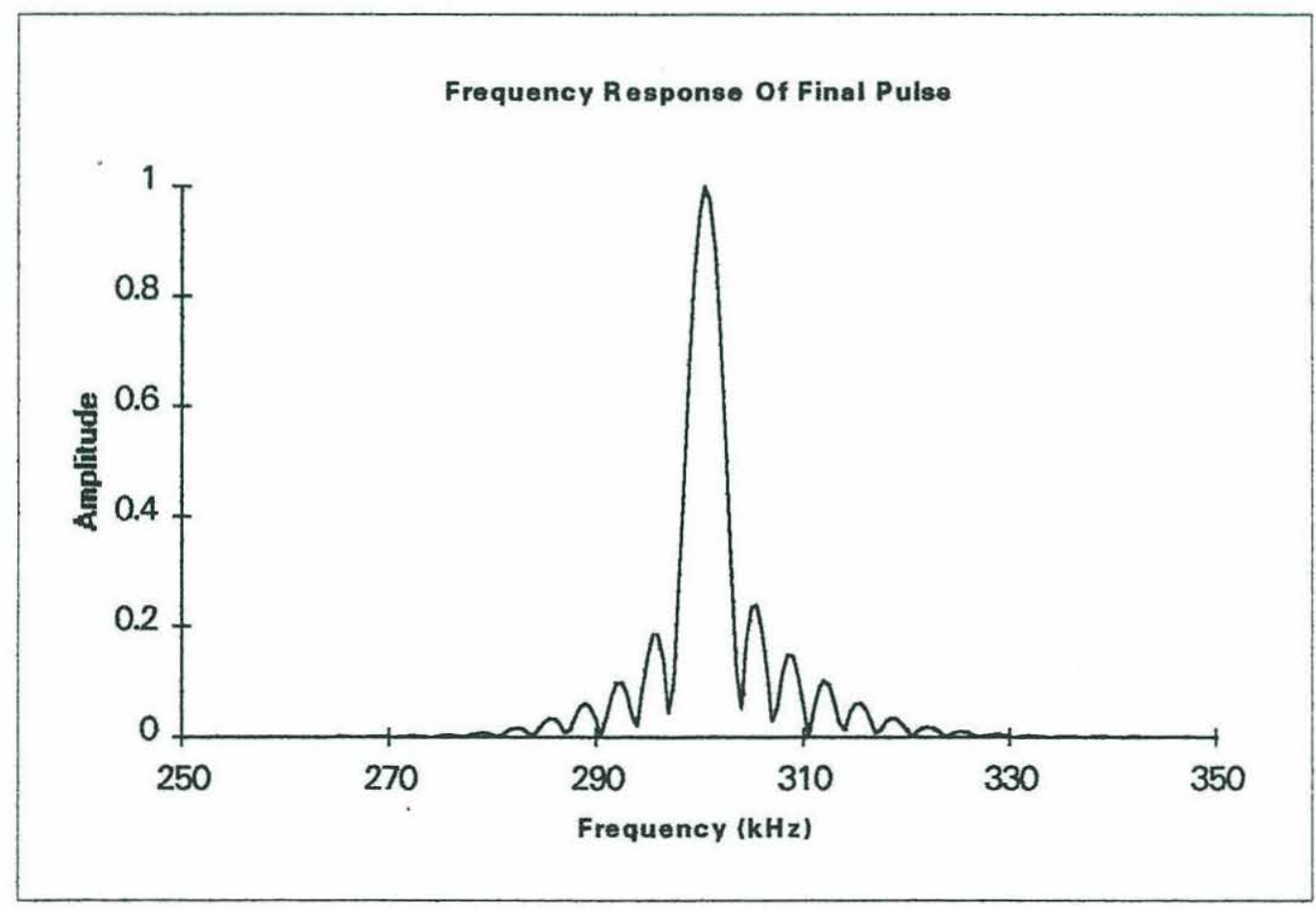

Fig 5.42 Final version of the returned pulse after analog processing. 


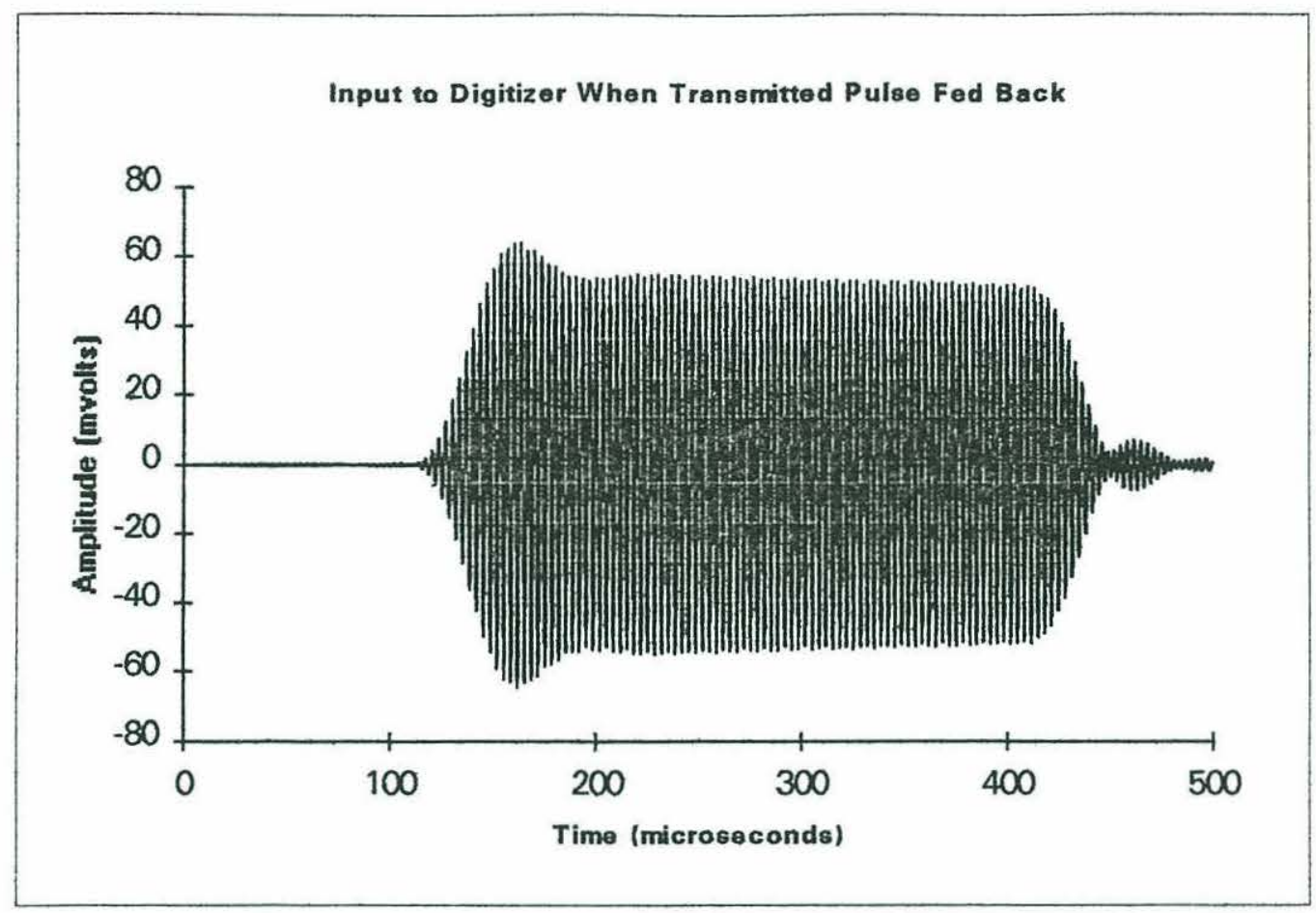

Fig 5.43 Final signal before sampling when a scaled version of the transmitted pulse is used as a test input.

received from the water column in a data set. Except for a small amount of reverberation from biologics and other sources, the expected return amplitude is zero. Thus the average return can be considered to be the sample and hold bias. As of this writing, a reliable data set was not yet available to perform this analysis.

\subsection{Summary}

The DSL-300 sonar can be analytically modeled as a set of cascaded filters. The model can be divided into two parts: a transmitter section and a receiver section. 
The transmitter section consists of a $300 \mathrm{kHz}$ carrier gated by a square pulse envelope. This signal is fed into a transducer modeled as an RLC oscillator, with component values chosen to match the measured resonant frequency and $\mathrm{Q}$. The resulting pulse is found to match the observed pulse, with the exception of a parasitic oscillation caused by the transformer leakage reactance and the transducer's shunt capacitance. This oscillation can be separately added to the model to produce the final result.

The receiver section consists of a network of flat gain and finite delay elements, which do not affect the final pulse shape, and a transducer, bandpass filter, and compensating filter which have important effects. The receiving transducer response can be modeled by using the component values determined from the transmitter along with the shunt capacitance and resistance determined from the measured impedance circle and discrete circuit elements. The bandpass filter is designed as a three-pole-pair butterworth filter with known parameters. The compensating filters are easily modeled as RC lowpass filters.

This completes the MATLAB model of the DSL $300 \mathrm{kHz}$ forward scanning sonar. In the next chapter, I discuss how this information can be used to remove some of the corrupting effects from the data. 


\section{Chapter 6}

\section{Inverse Modeling}

\subsection{Introduction}

It is desirable to use the results developed in Chapter 5 to remove the corrupting effects of the system from actual data. There are, of course, limits on our ability to do this, since data set to zero by a bandpass filter cannot be recovered. It is also impossible to achieve a perfectly ideal response in the passband due to the constraints of a finite sampling rate. It is still possible to improve the data in the passband by inverse filtering.

An inverse system function can normally be constructed by taking the reciprocal of the original system function. This process will be complicated by the presence of zeros in the original function, since they will become poles of the inverse function. To deal with this, the inverse function must be modified to ensure that it does not posses any singularities in its frequency response. Causality will not be a concern here, because the processing need not be done in real time.

Three elements in the system have the potential for causing a serious corrupting effect on the return: the transducer, the bandpass filter, and the phase compensating filters. Each of these elements is considered in the sections below. 


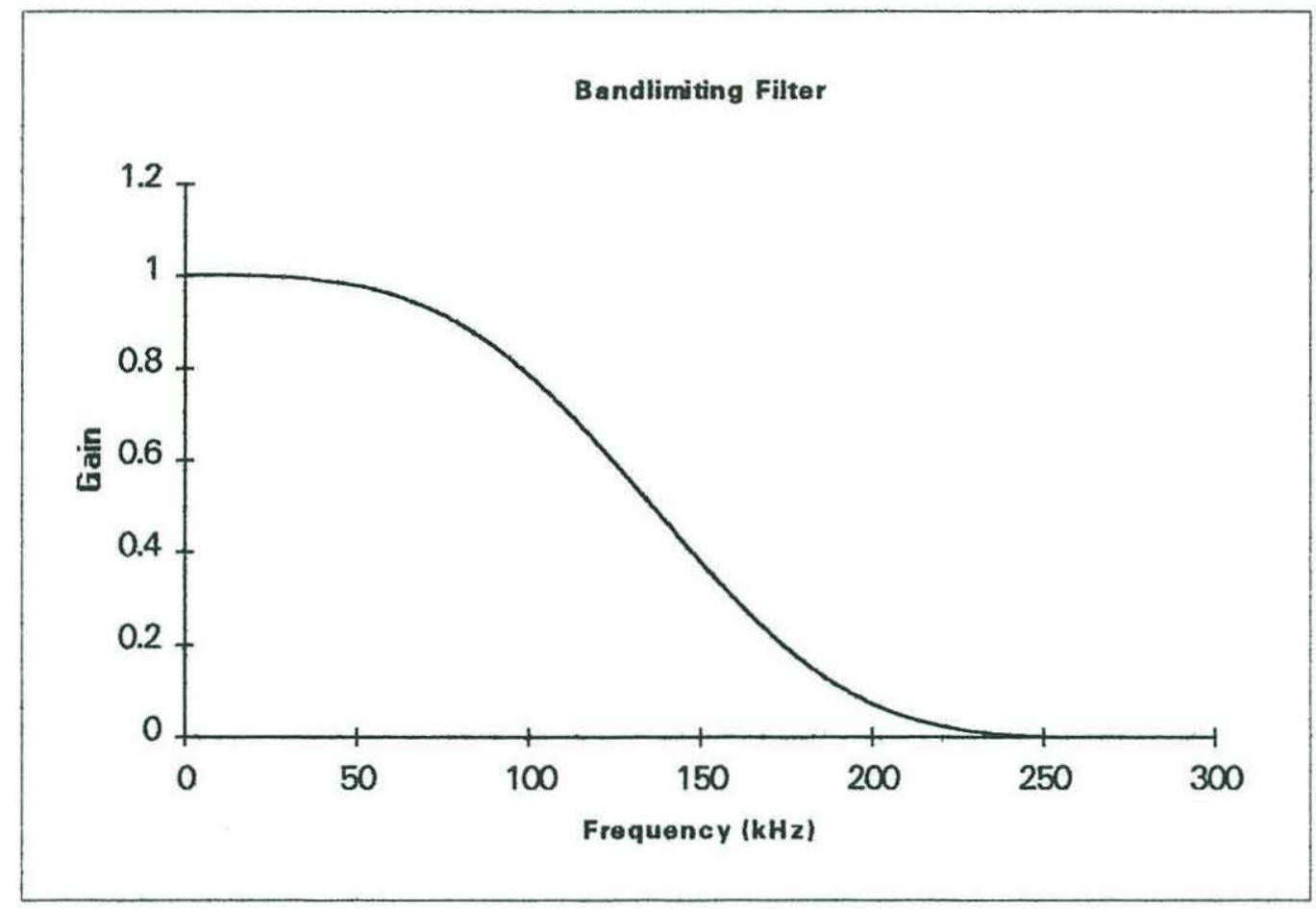

Fig 6.1 Magnitude of a band-limiting filter to ensure stability of the inverse response. Values near 300 $\mathrm{kHz}$ have been forced to zero to ensure pole cancelation. The gradual taper of this filter ensures that no spurious oscillations are introduced.

\subsection{The Transducer}

The receiving response of the transducer, Htr, is described by Equations 5.15 through 5.17. The reciprocal of $\mathrm{Htr}_{\mathrm{tr}} \mathrm{Gtr}$, expressed in terms of $s$ and shifted to baseband, is given by:

$$
\mathrm{Gtr}_{\mathrm{t}}=\frac{\mathrm{a} 4 \mathrm{~s}^{4}+\mathrm{a}_{3 s^{3}}+\mathrm{a}_{2} \mathrm{~s}^{2}+\mathrm{a}_{1} \mathrm{~s}+\mathrm{a} 0+j\left(\mathrm{~b}_{3} \mathrm{~s}^{3}+\mathrm{b}_{2} \mathrm{~s}^{2}+\mathrm{b}_{1} \mathrm{~s}+\mathrm{b}_{0}\right)}{\mathrm{c} 2 \mathrm{~s}^{2}+\mathrm{c} 0}
$$


Appropriate coefficients are given by Table 6.1. This function has a poles on the imaginary axis at $+/-300 \mathrm{kHz}$. It will be stable and well behaved as long as we stay far away from these points. If we limit our inverse processing to the $30-\mathrm{kHz}$ wide bandwidth of the bandpass filter this will not present a problem. To accomplish this, Equation 6.1 will be passed through a tapered filter with linear phase before being applied to the signal. This filter, Gwin, is shown if Fig 6.1. Equation 6.1 can be expressed in polar form:

$$
\begin{gathered}
\text { Gtr(mag) }=\quad\left(\frac{a f^{8}+a 7 f^{7}+a 6 f^{6}+a f^{5}+a 4 f^{4}+a 3 f^{3}+a 2 f^{2}+a 1 f+a 0}{c 4 f^{4}+c 2 f^{2}+c 0}\right) 1 / 2 \\
\text { Gtr(phase) }=\tan ^{-1}\left(\frac{a 3 f^{3}+a 2 f^{2}+a 1 f+a 0}{c 4 f^{4}+c 3 f^{3}+c 1 f+c 0}\right) \text { (b) }
\end{gathered}
$$

This response, multiplied by $\mathrm{G}_{\text {win, }}$, is illustrated in Figs 6.2 and 6.3. Fig 6.4 demonstrates the result of filtering a square pulse with the transducer model followed by inverse filtering by Equation 6.2 .

In order to make these results useful for processing the real data, the above results need to be translated into a set of discrete time filter coefficients at an appropriate sampling rate. This is most easily accomplished by using MATLAB to generate the reciprocal frequency response of the filter described by Equation 5.15, which is centered around $300 \mathrm{kHz}$. This function is passed through a square bandpass filter and transformed into the time domain, where it is sampled via the quadrature downsampling process. This will produce two sequences of numbers, gI and ge, which can be convolved with the in-phase and quadrature components of the data to produce the desired filtering effect: 


\section{Equation 6.1}

$$
\text { a0 }
$$

a1

a2

a3

a4

a5

a6

a7

a8

b0

b1

b2

b3

c0

c1

c2

c3

c4
$3.8 \times 10^{12}$

37.1

9.8

$2.9 \times 10^{-7}$

$2.5 \times 10^{-12}$
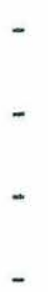

$-1.0 \times 10^{10}$

$3.3 \times 10^{7}$

0.6

$9.3 \times 10^{-6}$

$3.6 \times 10^{12}$

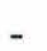

1.0

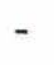

$-$
Equation 6.2a

$8.8 \times 10^{9}$

$-1.0 \times 10^{9}$

$2.7 \times 10^{7}$

$1.1 \times 10^{4}$

$5.8 \times 10^{2}$

1.0

$3.3 \times 10^{-3}$

$1.1 \times 10^{-5}$

$1.0 \times 10^{-8}$

-

-

$8.1 \times 10^{9}$

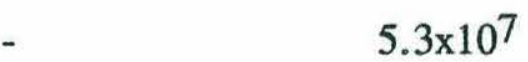

$-1.8 \times 10^{5}$

-

1.0
Equation 6.2b

$-2.6 \times 10^{6}$

59.8

$6.1 \times 10^{3}$

18.5
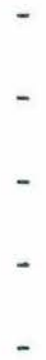

$9.8 \times 10^{8}$

$5.3 \times 10^{7}$

$5.9 \times 10^{2}$

1.0

Table 6.1 Coefficients for Equations 6.1 and 6.2. 


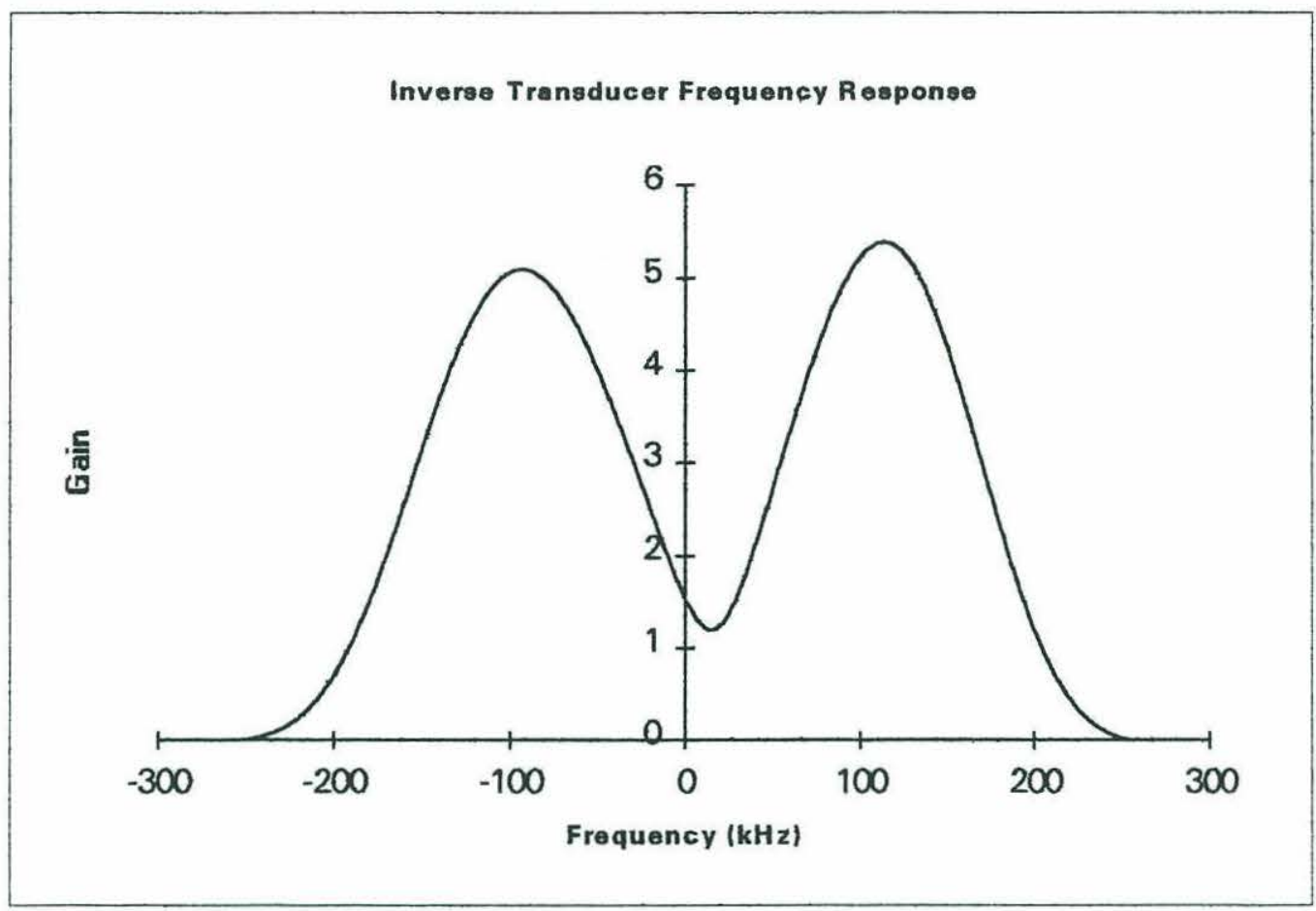

Fig 6.2 Frequency response of a filter to correct for the corrupting effects of the transducer.

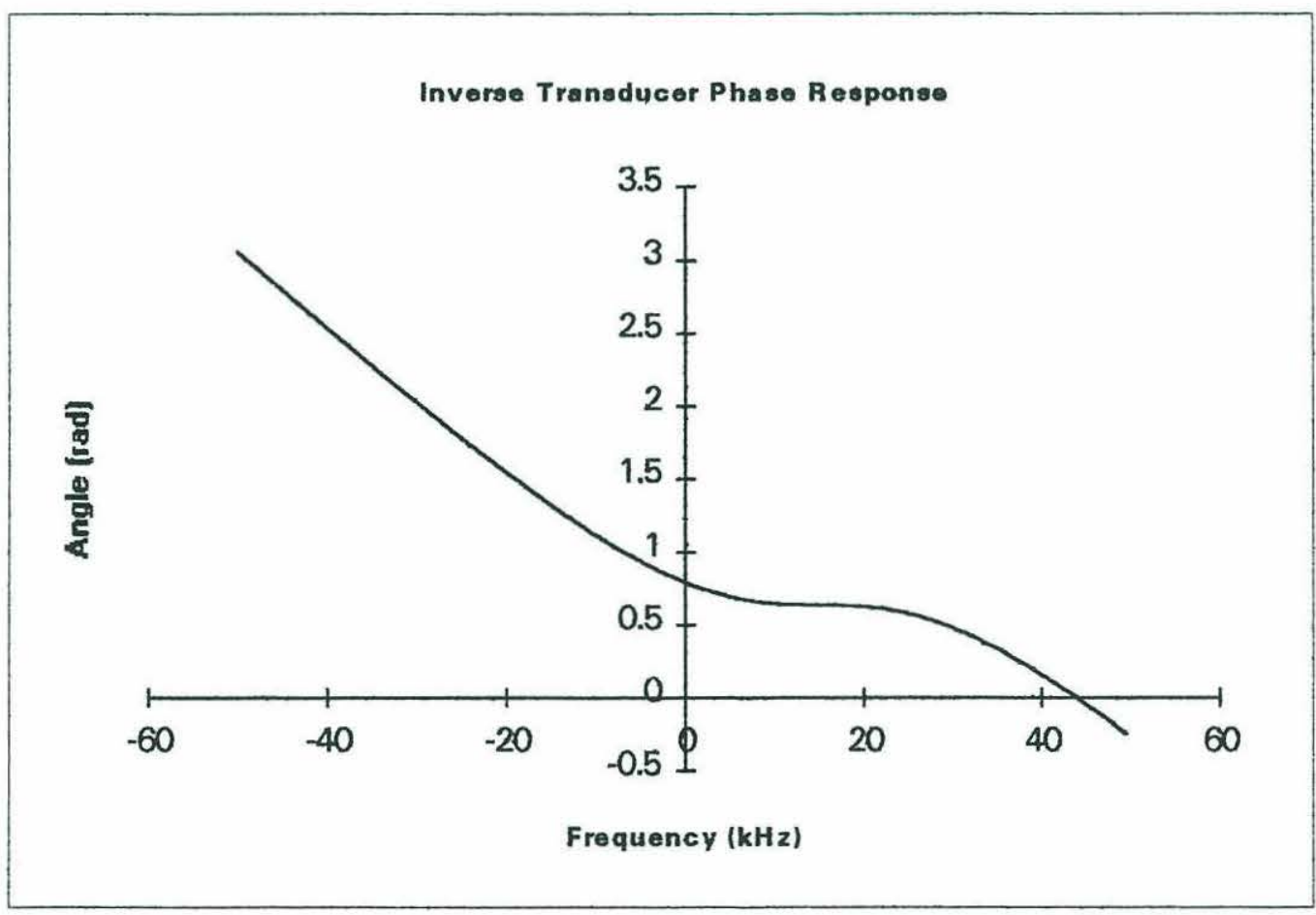

Fig 6.3 Phase response corresponding to Fig 6.2. 

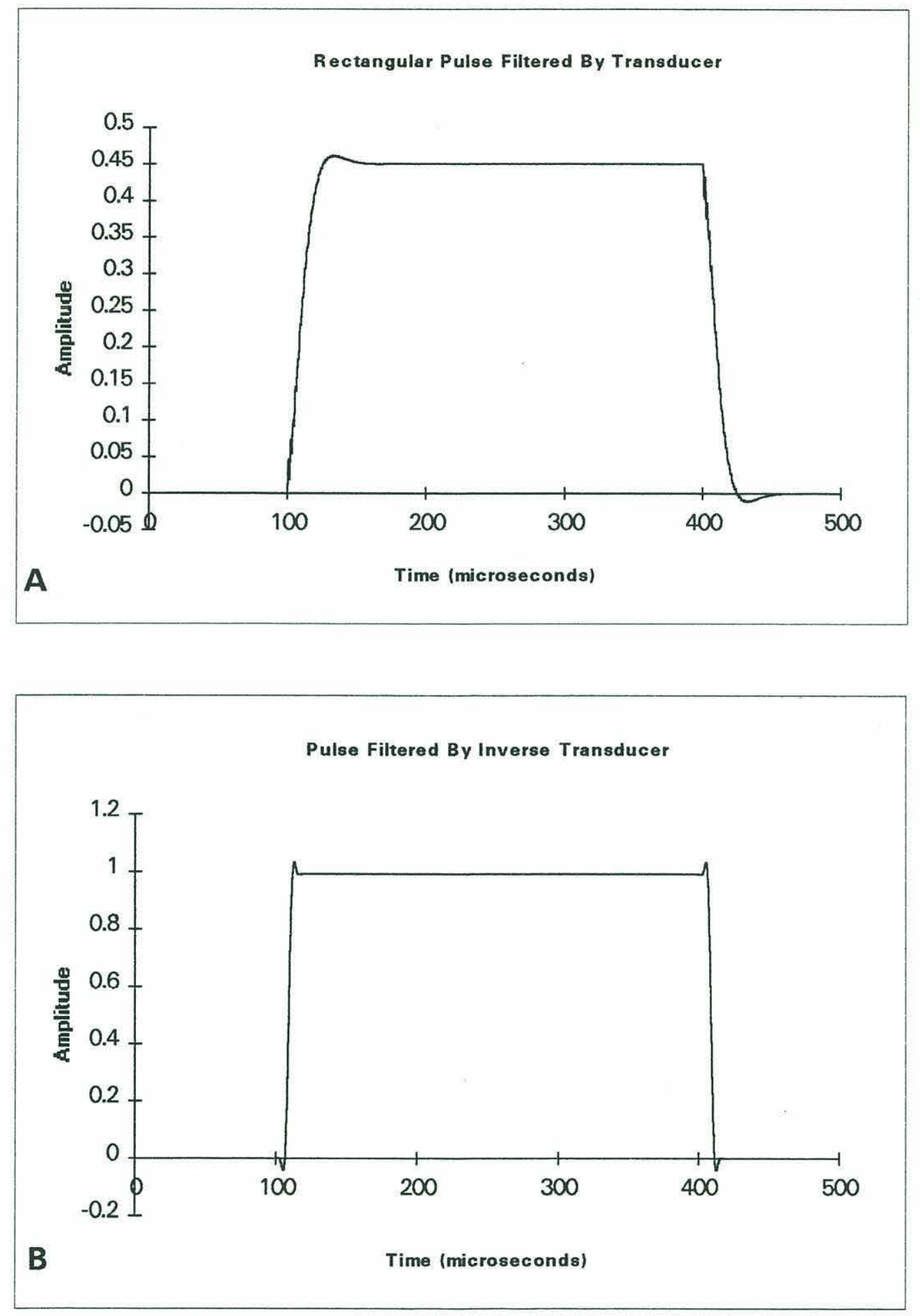

Fig 6.4 Result of filtering a rectangular pulse by the model transducer (A) followed by filtering by the inverse transducer (B). 


$$
\begin{aligned}
\mathrm{XIF}(\mathrm{t})+j \mathrm{XQF}(\mathrm{t}) & =(\mathrm{gI}(\mathrm{t})+j \mathrm{gQ}(\mathrm{t})) *\left(\mathrm{XI}_{\mathrm{I}}(\mathrm{t})+j \mathrm{XQ}(\mathrm{t})\right) \\
& =\left(\mathrm{gI}(\mathrm{t}) * \mathrm{XI}_{\mathrm{I}}(\mathrm{t})-\mathrm{gQ}(\mathrm{t}) * \mathrm{XQ}_{(\mathrm{t})}\right)+j\left(\mathrm{gI}(\mathrm{t}) * \mathrm{XQ}_{\mathrm{Q}}(\mathrm{t})+\mathrm{gQ}(\mathrm{t}) * \mathrm{XI}_{\mathrm{I}}(\mathrm{t})\right)
\end{aligned}
$$

$\mathrm{XI}_{\mathrm{I}}$ and $\mathrm{XQ}_{\mathrm{Q}}$ represent the in-phase and quadrature components of the actual data, while $\mathrm{X}_{\mathrm{IF}}$ and $\mathrm{X}_{\mathrm{QF}}$ are the components after filtering.

Table 6.2 gives the appropriate sequences to apply to the DSL 300 data to simulate the inverse transducer, assuming a $10 \mathrm{kHz}$ complex sampling rate.

Time (microsec)

-1000
-900
-800
-700
-600
-500
-400
-300
-200
-100
0
100
200
300
400
500
600
700
800
900

In-Phase

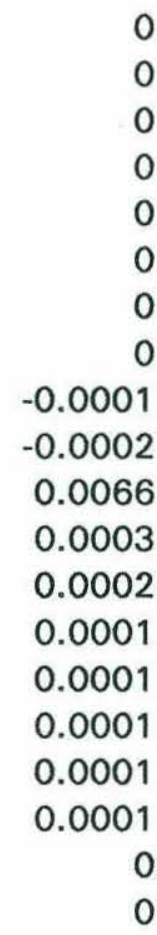

0

0

\section{Quadrature}

0

0

0

0

0.0002

$\begin{array}{ll}0 & 0.0002\end{array}$

0
0.0003

0.0005

0.0011

0.0188

$-0.0011$

$-0.0005$

$-0.0003$

$-0.0002$

$-0.0002$

$-0.0001$

$-0.0001$

\section{0}

0

0.0001

0.0001

0

0
0.0001

Table 6.2 Array to convolve with the raw sonar data to simulate the inverse transducer. 


\subsection{The Bandpass Filter}

Although it is impossible to recover the frequencies set to zero by the bandpass filter, it is possible to modify the passband characteristics of the Butterworth filter to approximate an ideal filter. The procedure is identical to that used for the transducer.

The equation for the Butterworth bandpass filter was given by Equation 5.26. This must be shifted to baseband, where it will become a lowpass filter, and inverted:

$$
\mathrm{Gbp}_{\mathrm{b}}=\mathrm{a}_{2} \mathrm{f}^{2}+\mathrm{alf}_{1}+\mathrm{a}_{0}+j\left(\mathrm{~b}_{3} \mathrm{f}^{3}+\mathrm{b}_{2} \mathrm{f}^{2}+\mathrm{b}_{1} \mathrm{f}+\mathrm{b}_{0}\right)
$$

In polar form:

$$
\begin{aligned}
& G b(\text { mag })=\left(a 6 f^{6}+a f^{5}+a 4 f^{4}+a 3 f^{3}+a 2 f^{2}+a 1 f+a 0\right)^{1 / 2} \\
& \text { Gbp(phase) }=\tan ^{-1}\left(\frac{a 3 f^{3}+a 2 f^{2}+a 1 f+a 0}{c 2 f^{2}+c 1 f+c 0}\right)
\end{aligned}
$$

Coefficients are given by Table 6.3 . These responses, once again band-limited by the square filter, are plotted in Figs 6.5 and 6.6. Fig 6.7 shows the result of forward and backward filtering of a square pulse.

Coefficients to convolve with the real data arrays are generated as before, with the results given in Table 6.4 . 


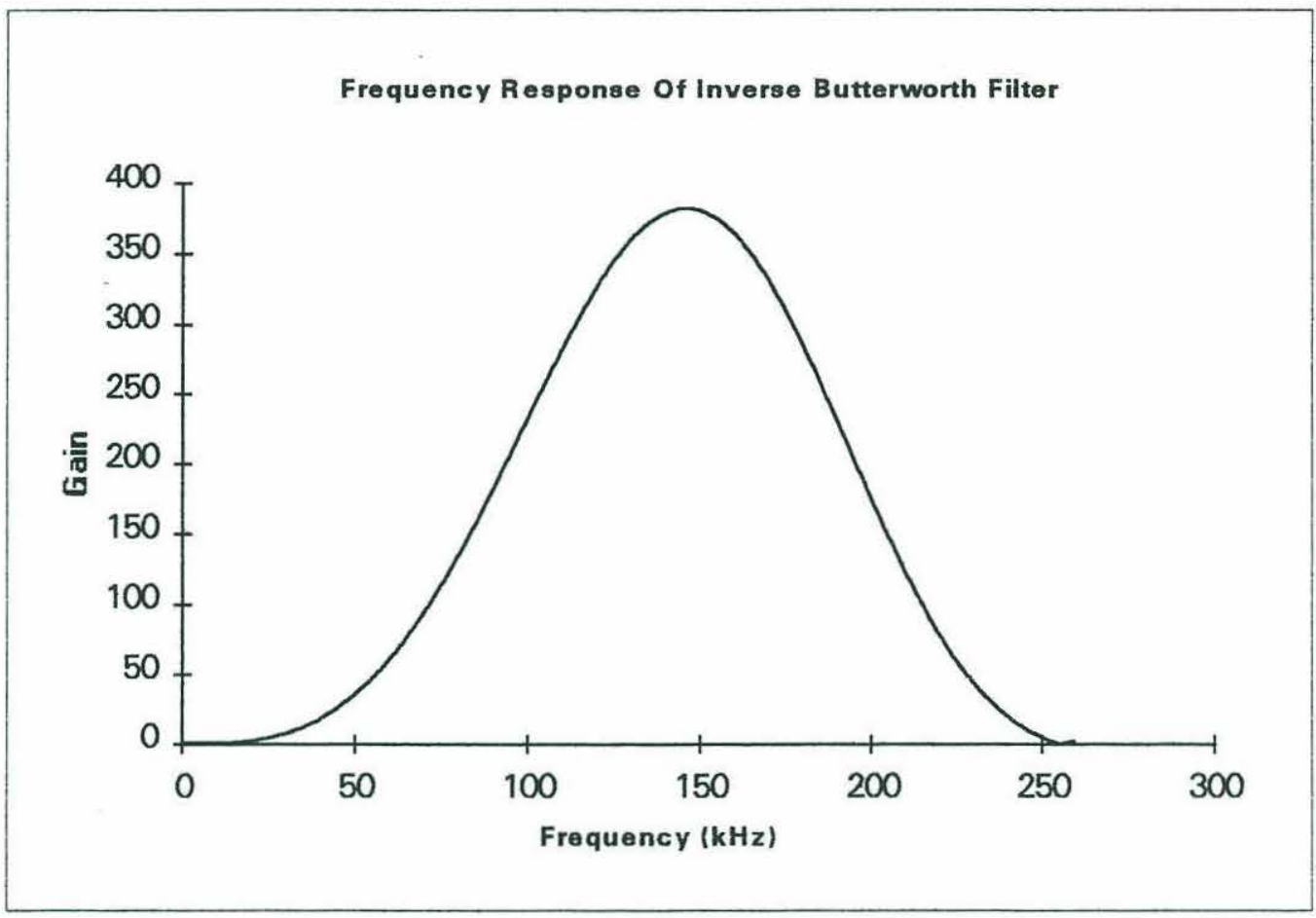

Fig 6.5 Frequency response of a filter to reverse the effects of the Butterworth filter in the passband. Negative half is symmetric.

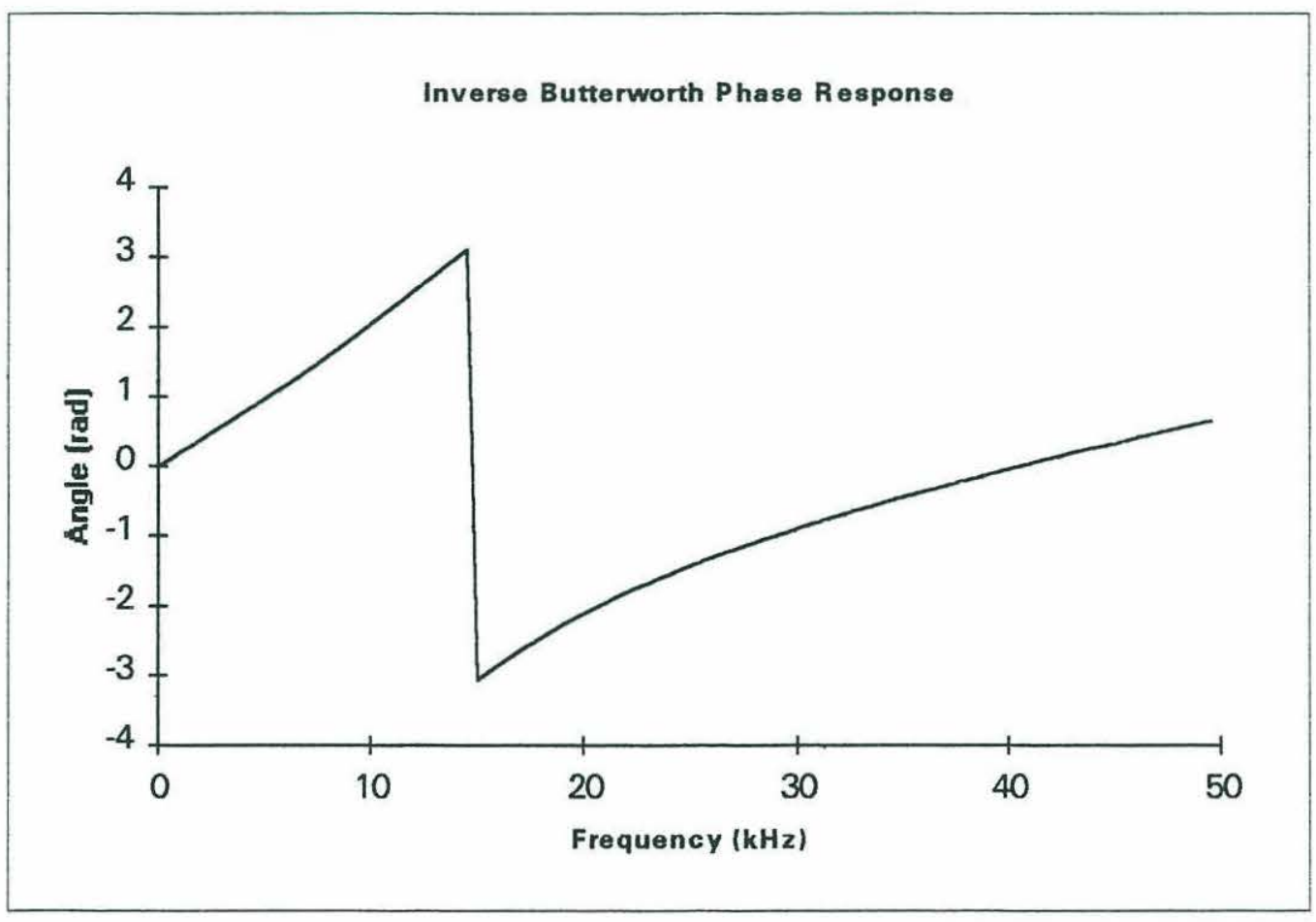

Fig 6.6 Phase response corresponding to Fig 6.5. Negative half is anti-symmetric. 

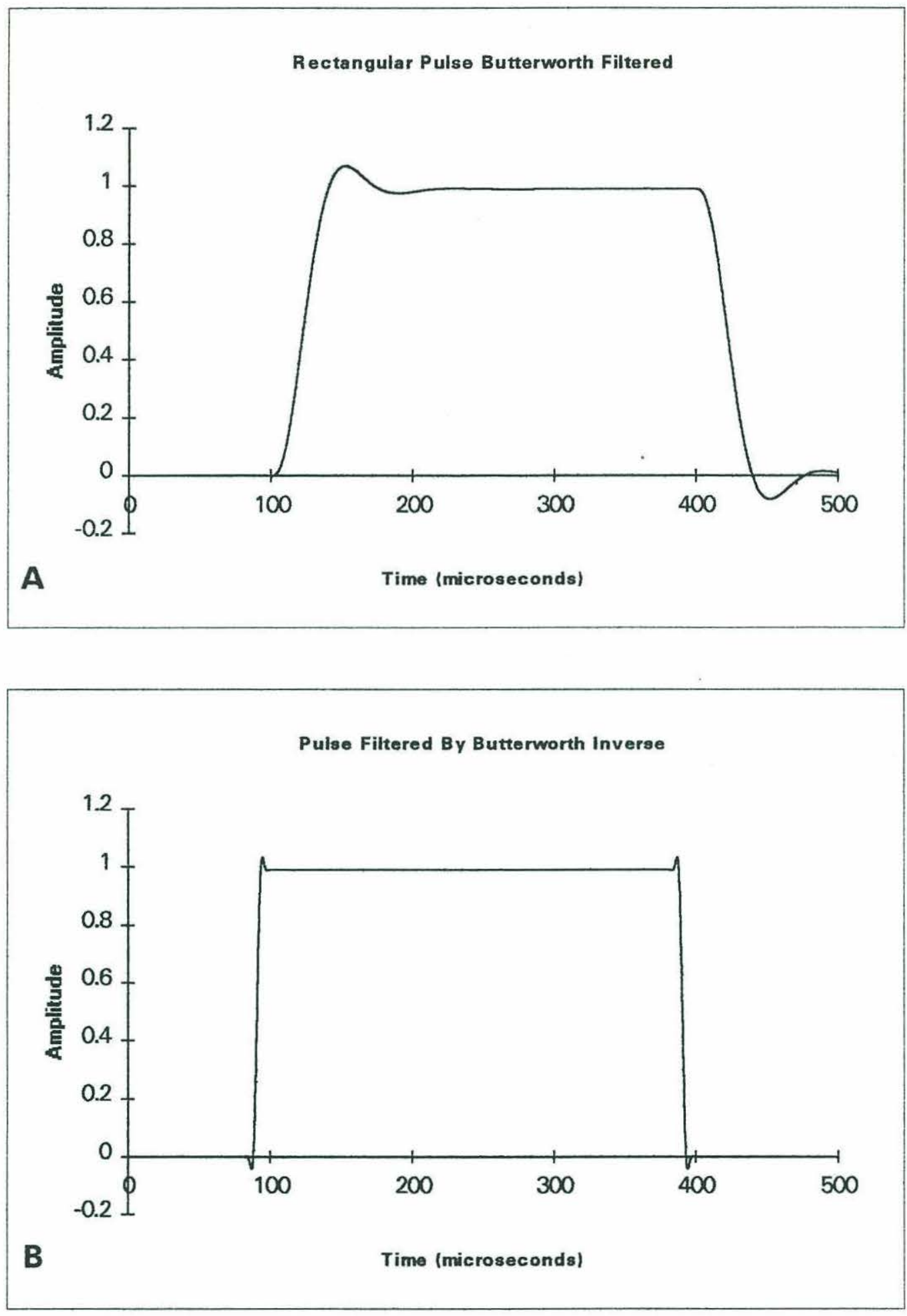

Fig 6.7 Result of filtering a rectangular pulse by the Butterworth filter (A) followed by inverse filtering (B). 


\section{Equation 6.4}

a0

a1

a2

a3

a4

a5

a6

b0

b1

b2

b3

c0

c1

c2

0.59

$-0.16$

$-1.8 \times 10^{-2}$
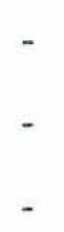

$-$

0.81

0.17

$-7.1 \times 10^{-3}$

$-5.9 \times 10^{-4}$
Equation 6.5a

1.0

$8.5 \times 10^{-2}$

$2.3 \times 10^{-2}$

$2.3 \times 10^{-3}$

$1.5 \times 10^{-4}$

$8.4 \times 10^{-6}$

$3.5 \times 10^{-7}$

Table 6.3 Coefficients for Equations 6.4 and 6.5.

\subsection{The Phase-Compensating Filters}

It is obviously not desirable to undo the delay characteristic of the compensating filters but it is possible to adjust their amplitude to unity in the passband. The filter response is given by Equations 5.30 and 5.31. Setting the phase angle to zero yields an 


\section{Time (microsec)}

$-1000$

$-900$

$-800$

$-700$

$-600$

$-500$

$-400$

$-300$

$-200$

$-100$

0

100

200

300

400

500

600

700

800

900
In-Phase

$-0.0221$

$-0.1441$

$-0.2454$

$-0.3104$

$-0.3248$

$-0.2645$

$-0.1294$

0.1129

0.5468

1.6147

1.0869

$-1.6699$

$-0.6014$

$-0.16$

0.0929

0.2401

0.3083

0.3113

0.2609

0.1699

\section{Quadrature}

$-0.1608$

$-0.2477$

$-0.2926$

$-0.2859$

$-0.221$

$-0.0782$

0.1217

0.4056

0.8382

1.781

$-18.9464$

$-1.1016$

$-0.1695$

0.1665

0.3174

0.3653

0.3417

0.2664

0.157

0.0308

Table 6.4 Arrays to simulate the inverse Butterworth filter.

\begin{tabular}{|llllll|}
\hline $\mathbf{a 1 2}$ & $\mathbf{a 1 1}$ & $\mathbf{a 1 0}$ & $\mathbf{a} 22$ & $\mathbf{a 2 1}$ & $\mathbf{a 2 0}$ \\
0.45 & $-2.7 \times 10^{-2}$ & $2.0 \times 10^{5}$ & 0.95 & $-5.7 \times 10^{2}$ & $1.5 \times 10^{6}$ \\
\hline $\mathbf{c 1 2}$ & $\mathbf{c 1 1}$ & $\mathbf{c 1 0}$ & $\mathbf{c 2 2}$ & $\mathbf{c 2 1}$ & $\mathbf{c 2 0}$ \\
$1.8 \times 10^{8}$ & $-1.1 \times 10^{11}$ & $8.1 \times 10^{13}$ & $4.2 \times 10^{7}$ & $-2.6 \times 10^{10}$ & $6.8 \times 10^{13}$ \\
\hline
\end{tabular}

Table 6.5 Coefficients for Equation 6.6.

entirely real filter. Inverting the magnitude from 5.31, substituting actual component values, and weighting in accordance with the gain of the branches yields: 


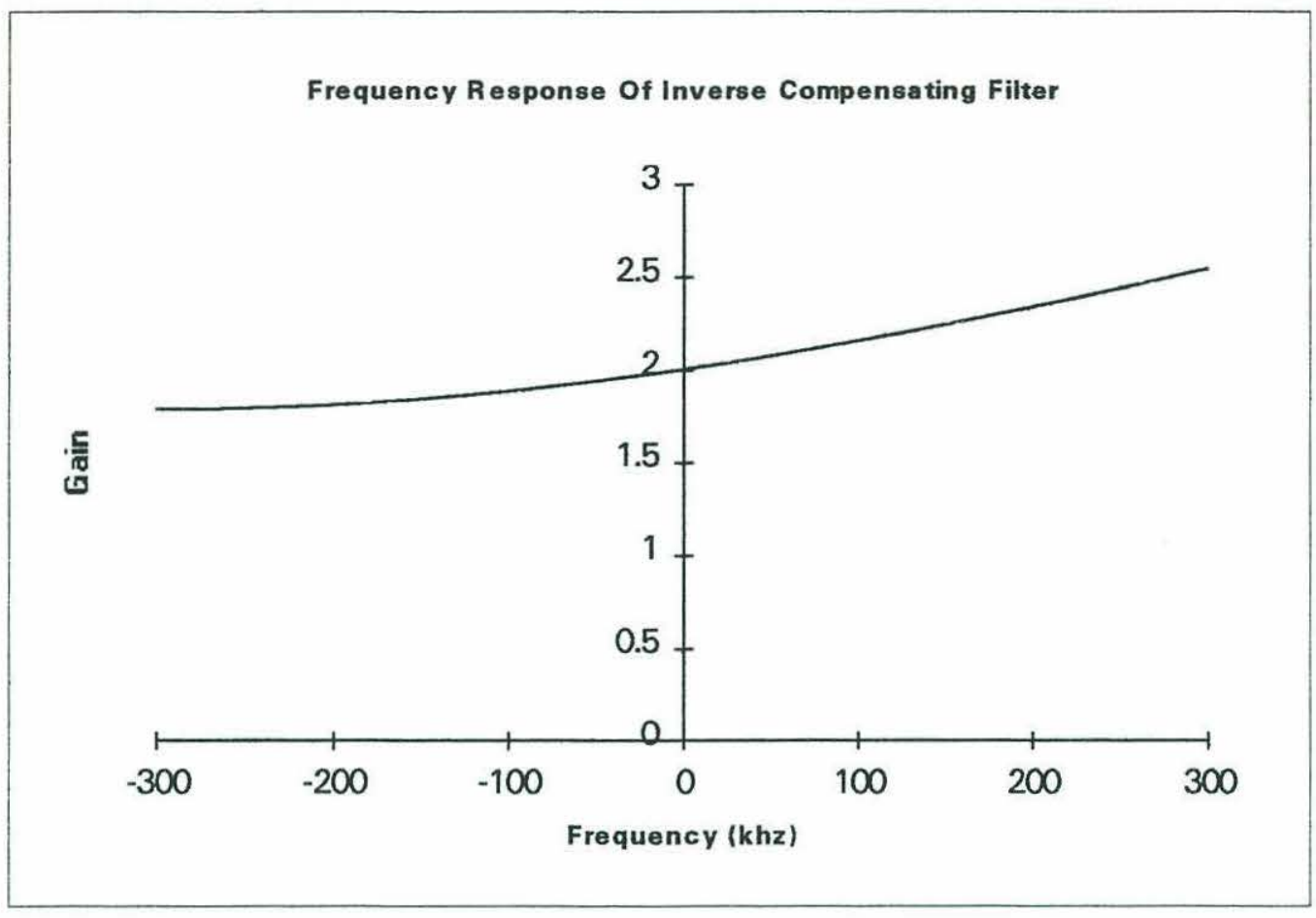

Fig 6.8 Zero-phase compensating-filter inverse function.

$$
\begin{aligned}
G_{p c} & =\frac{a_{12} f^{2}+a 11 f+a 10}{\left(c_{12} f^{2}+c 11 f+c_{10}\right)^{1 / 2}} \\
& +\frac{a 22 f^{2}+a 21 f+a 20}{\left(c_{22} f^{2}+c_{21} f+c_{20}\right)^{1 / 2}}
\end{aligned}
$$

Coefficients are given by Table 6.5. This equation is plotted in Fig 6.8. Once again, it is multiplied by the rectangular filter to limit the bandwidth. Due to it's broad and relatively flat characteristics, this filter will have no noticeable effect on the model post. In fact, the corresponding coefficient array reduces to an impulse at the origin for the $10 \mathrm{kHz}$ sampling rate. From this I conclude that the compensating filters should be ignored when implementing an inverse model. 


\subsection{Summary}

The most serious corrupting effects of the receiver are caused by the transducer, bandpass filter, and phase compensating filters. Each of these components can be represented by a filter which is specified mathematically in Chapter 5 as a rational function. An inverse filter can be constructed by taking the reciprocal of these functions and passing them through a suitable frequency window to eliminate any unstable poles. The process will not exactly reproduce the original pulse because frequency components originally set to zero cannot be recovered. It is possible to make substantial improvements, as the plots in this chapter illustrate.

The inverse filters can be applied to actual data by simulating the quadrature sampling process on MATLAB. This will generate a set of in-phase and quadrature coefficients which can be convolved with the data as specified in Equation 6.3.

To conclude this chapter, it should be noted that the sample/hold biases calculated in Chapter 5 can be subtracted directly from the raw data to improve the phase calculation's accuracy. 


\section{Chapter 7}

\section{Summary, Conclusions, and Recommendations}

The purpose of this thesis was to construct a mathematical model of the DSL 300 $\mathrm{kHz}$ sonar system. To achieve this goal, a generic sonar system was developed to determine what the essential components of any system are. Each of these components, both electrical and mechanical, were analyzed to determine how they could be simulated mathematically. The results were then made specific to the WHOI/APL system. Finally, a technique was developed to inverse filter the actual sonar data to remove some of the system-dependent effects.

It was possible to match the observed characteristics reasonably well, with some limitations. The most difficult component to model correctly was the transducer, because of the limited measurement accuracy (up to $+/-1.5 \mathrm{~dB}$ ) of the frequency response curve. The transmit response curve determined at APL was used to calculate equivalent values of inductance and capacitance for the model transducer. The effects of this error can be seen by comparing the receive value of $Q$ calculated from the model response (Fig 5.22) with that determined from the actual receiver plot (Fig 4.7). The model predicts a value of 9.5 , but measurements indicate 7.7 . The predicted value is within the range of possible values expected if measurement error is considered. It should be possible to obtain more accurate values by conducting a careful impedance verses frequency measurement of the transducer. 
A further limitation of the model is it's failure to predict the parasitic oscillation. This feature was added to separately to match the observed result. Since this phenomenon is associated with the leakage inductance of the coupling transformer, a detailed model of this component could be developed to produçe better results. A possible technique for this is discussed in Ref [19]. Such a model could also be applied to the isolation transformer at the receiver input, which was treated here as ideal.

The inverse modeling experiments demonstrate that it is possible to make corrections to a rectangular test pulse which had been corrupted by the system. The results were significant for the transducer and the band-limiting filter. Although a method was outlined for applying the corrections to real data, lack of an adequate data set has delayed demonstration of its actual utility. When such data is obtained, further refinements can be made to the technique. Calculation of the sample/hold bias will also become possible.

Despite its limitations, this model should prove useful for determining the actual shape, energy, and frequency content of both transmitted and received signals in the real world. Its results can be easily transported to other sonar systems by determining component parameters in the same fashion as demonstrated here. There will be some design differences between systems. Many transducers, for example, are deliberately connected to a shunt inductance to cancel the intrinsic shunt capacitance. It is important to examine the system carefully to determine if there are any other components which might alter the transducer response. For example, the $300 \mathrm{kHz}$ transducers considered here are very sensitive to the shunt capacitance, which is altered by the transmit/receive network. Others sonars may also use different types of transducers, such as magnetostrictive or electrostrictive, which require slightly different representations. Different types of band-limiting filters (such as elliptic or Chebyshev) 
are also common, along with different demodulation schemes. Some systems will include extra features, such as Time Varying Gain (TVG) and Automatic Gain Control (AGC). These will generally be easy to model because their response is given explicitly by the manufacturer.

When constructing inverse system models, care must be taken when selecting a stabilizing filter. As discussed in Chapter 6, these are required to eliminate poles outside the region of interest. Failure to properly control filter overshoot and sidelobes can lead to undesirable oscillations being imposed on the data. It may also be necessary to force the filter values to zero at a certain point, since even a very small gain can cause trouble in the vicinity of a pole.

It may be desirable to automate the inverse filtering process and allow it to proceed in real-time. If this is done, care must be taken to design causal filters. The inverse filters considered here can be approximated with a finite impulse response, allowing causality to be obtained by imposing a delay. 


\section{Appendix}

\section{MATLAB Macros For DSL-300 Model}

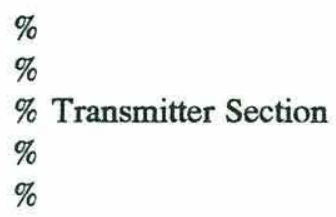

format long;

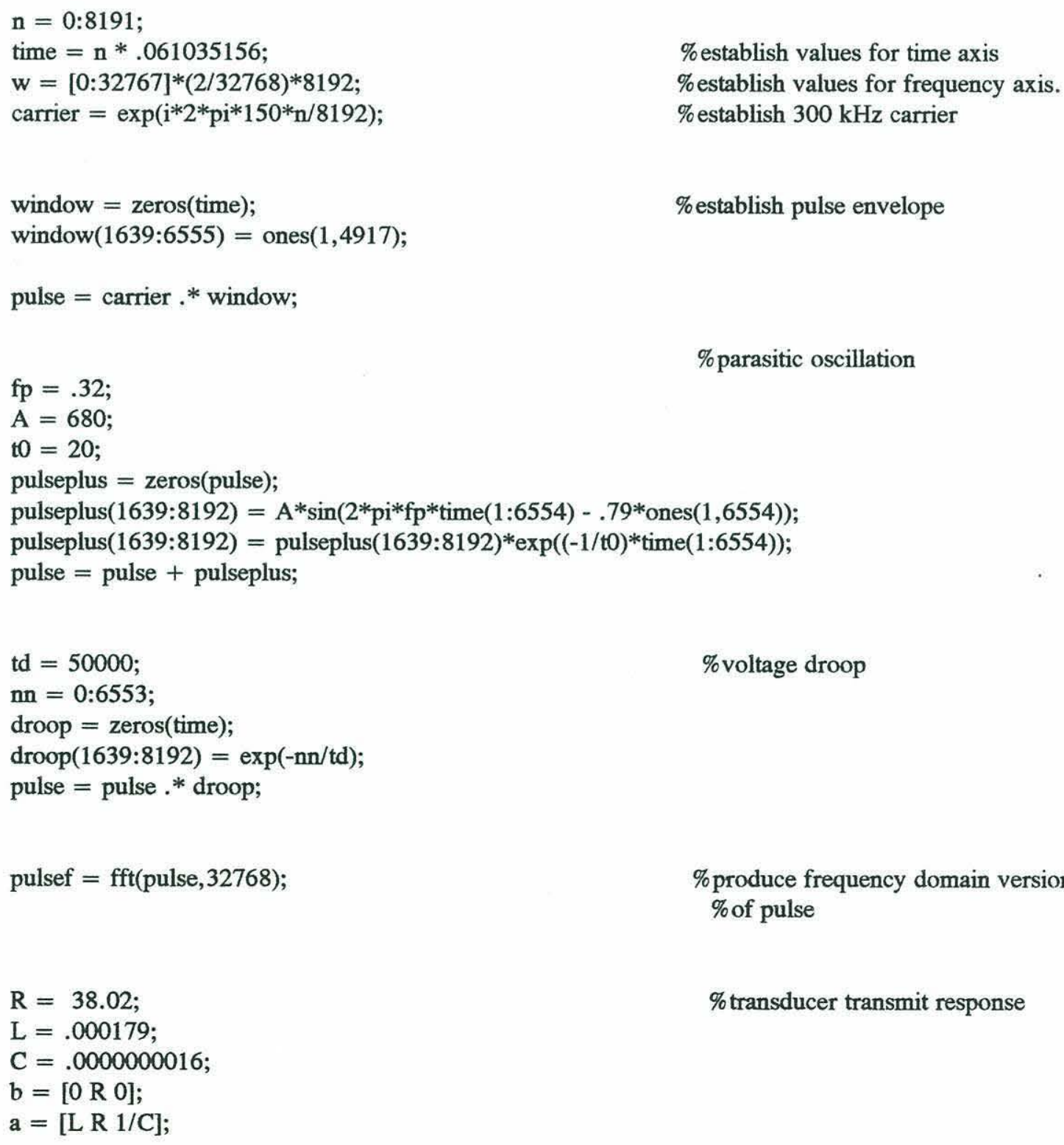

\%establish values for time axis

\%establish values for frequency axis. \% establish $300 \mathrm{kHz}$ carrier

\%establish pulse envelope

$\%$ parasitic oscillation 
$[\mathrm{r}, \mathrm{p}, \mathrm{k}]=$ residue $(\mathrm{b}, \mathrm{a})$;

$\mathrm{r}=.000000061 * \mathrm{r}$;

$\mathrm{p}=\exp (.000000061 * \mathrm{p})$;

$[\mathrm{b}, \mathrm{a}]=$ residuez $(\mathrm{r}, \mathrm{p}, \mathrm{k})$;

ducer $=$ freqz $(b, a, 32768$, 'whole' $)$;

ducer $=$ abs(ducer).$* \exp \left(-i^{*}\right.$ angle(ducer $\left.)\right) ; \quad$ \%make causal

ducer $=$ ducer';

ducerpulsef $=$ ducer.$^{*}$ pulsef;

ducerpulse $=$ ifft(ducerpulsef);

ducerpulse $=$ ducerpulse $(1: 8192)$;

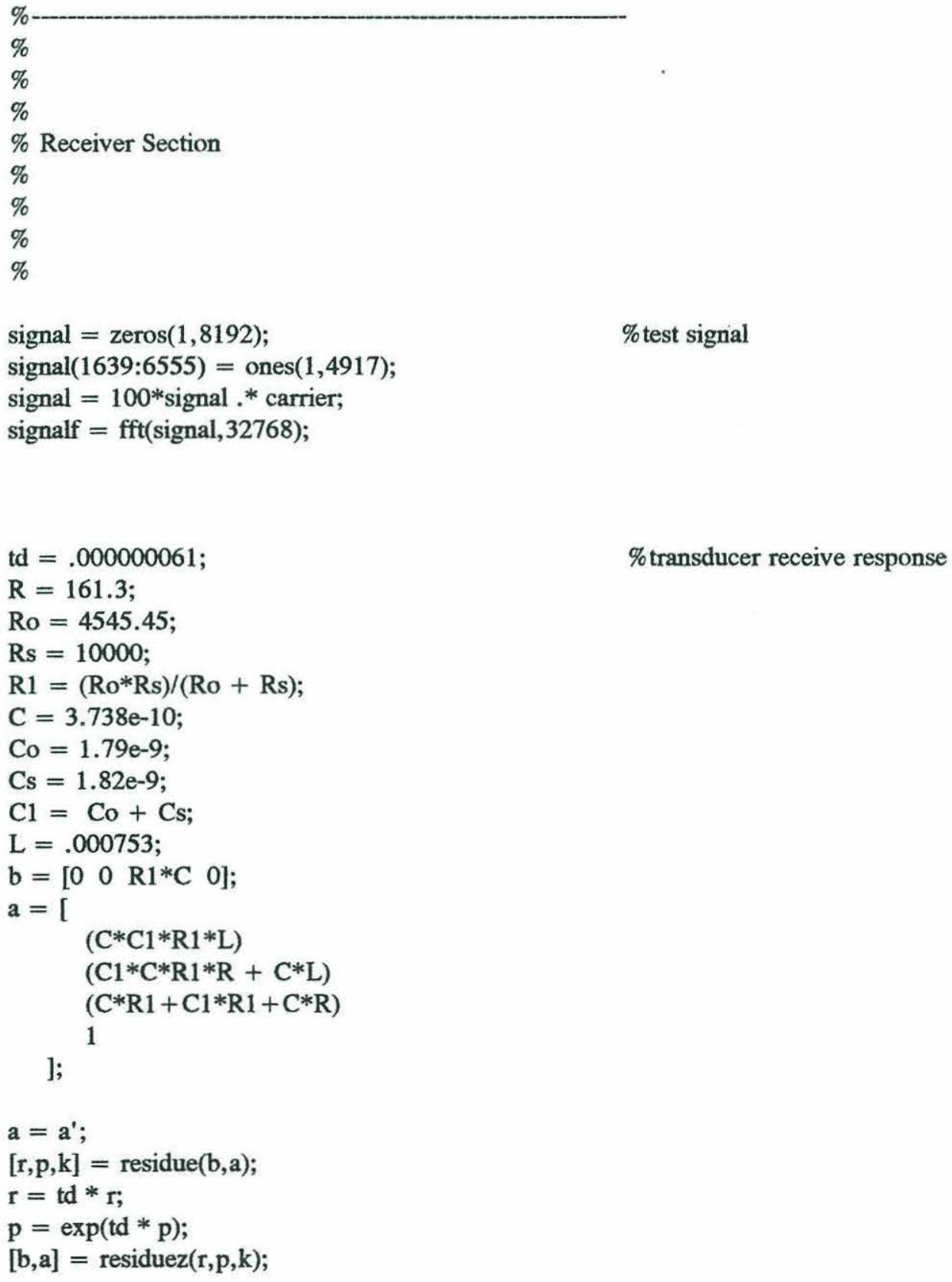




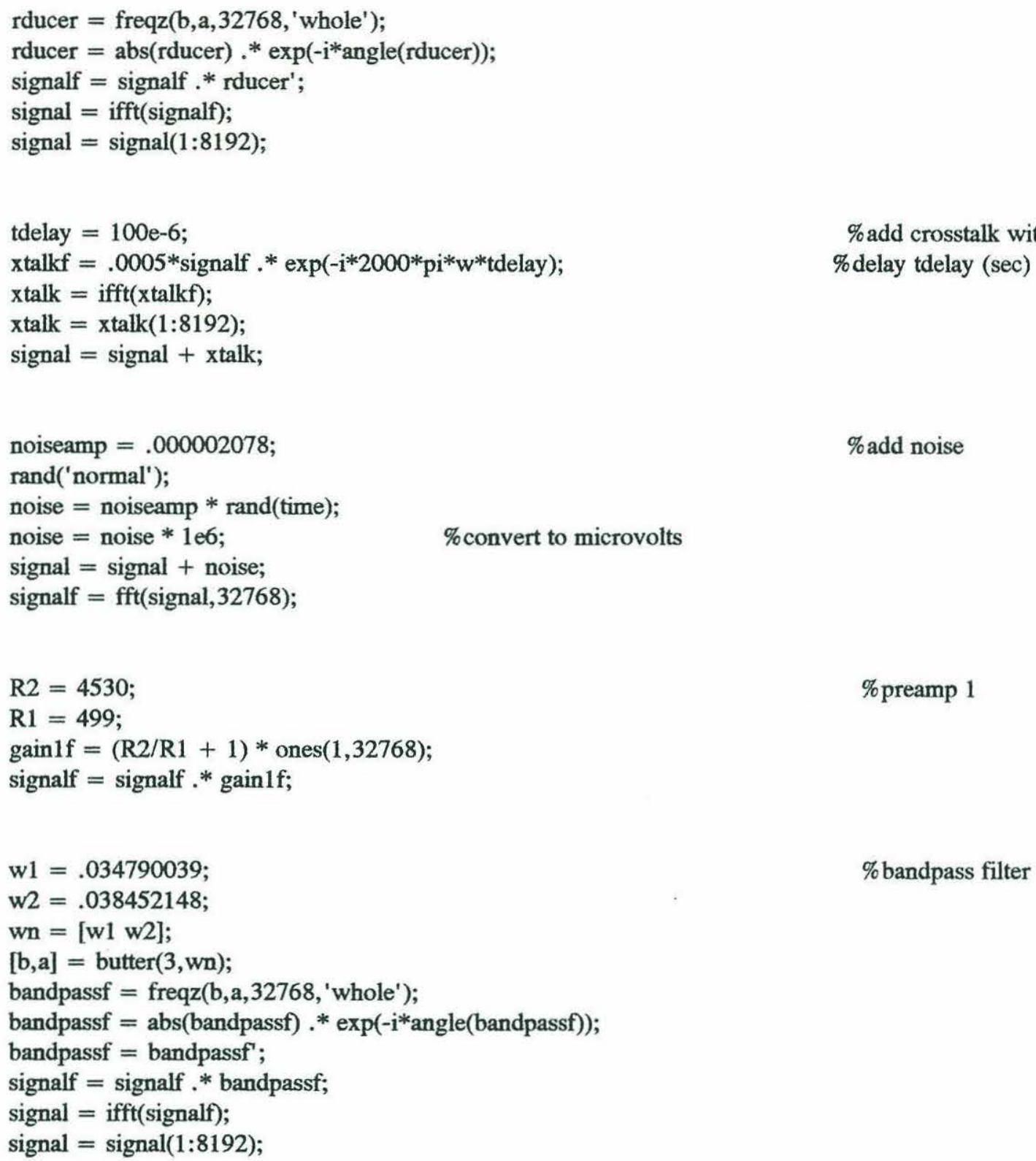
\%delay tdelay ( $\mathrm{sec})$

\%add noise

\% bandpass filter

\% add crosstalk with time 
delayf $=\exp \left(\mathrm{i}^{*} \mathrm{pi}^{*} \mathrm{w}^{*}\right.$ tdelay $)$

signalf $=$ signalf.$*$ delayf;

$\mathrm{R} 1=2000$

$\%$ comp $1(0 \mathrm{~dB})$

$\mathrm{R} 2=2000$

$\mathrm{C}=270 \mathrm{e}-12$;

$r=1 /(R 2 * C)$;

$\mathrm{p}=-2 * \mathrm{R} 1$

$\mathrm{p}=\exp (\mathrm{td} * \mathrm{p})$

$\mathrm{r}=\mathrm{td} * \mathrm{r}$;

$[\mathrm{b}, \mathrm{a}]=\operatorname{residuez}(\mathrm{r}, \mathrm{p}, \mathrm{k})$;

compof $=$ freqz $(b, a, 32768$, 'whole' $)$;

compOf $=$ abs(compOf $). * \exp \left(-\mathrm{i}^{*}\right.$ angle(comp0f $)$;

compOf $=$ compOf';

signalof $=$ signalf.$*$ compof;

signal0 $=$ ifft(signal0f);

signal0 $=\operatorname{signal0}(1: 8192)$;

$\mathrm{R} 2=4020$;

$\% \operatorname{amp} 1(20 \mathrm{~dB})$

$\mathrm{R} 1=499$;

gain20f $=(\mathrm{R} 2 / \mathrm{R} 1+1) *$ ones $(1,32768)$;

signal20f $=$ signalf.$*$ gain $20 \mathrm{f}$;

tdelay $=1.6 \mathrm{e}-7$

delay $20 \mathrm{f}=\exp (-\mathrm{i} * 2000 * \mathrm{pi} * \mathrm{w} *$ tdelay $)$;

signal20f $=$ signal2 $0 f . *$ delay $20 \mathrm{f}$;

$\%$ delay 2

$\mathrm{R} 1=2000$;

$\mathrm{R} 2=2000$;

$\mathrm{C}=130 \mathrm{e}-12$;

$r=1 /(R 2 * C)$;

$\mathrm{p}=-2 * \mathrm{R} 1$

$\mathrm{r}=\mathrm{td} * \mathrm{r}$

$\mathrm{p}=\exp (\mathrm{td} * \mathrm{p})$

$[\mathrm{b}, \mathrm{a}]=\operatorname{residuez}(\mathrm{r}, \mathrm{p}, \mathrm{k})$;

comp20f $=$ freqz(b, a, 32768, 'whole');

comp20f $=$ abs (comp20f).$* \exp \left(-\mathrm{i}^{*}\right.$ angle $($ comp20f $)$;

comp20f = comp20f';

signal20f $=$ signal2 $0 f . *$ comp $20 \mathrm{f}$;

signal20 = ifft(signal20f);

signal20 $=$ signal20(1:8192);

$\mathrm{R} 2=4530$;

R1 = 499;

gain40f = gain20f.$*((\mathrm{R} 2 / \mathrm{R} 1+1) *$ ones $(1,32768))$;

signal40f $=$ signalf.$*$ gain $40 \mathrm{f}$; 


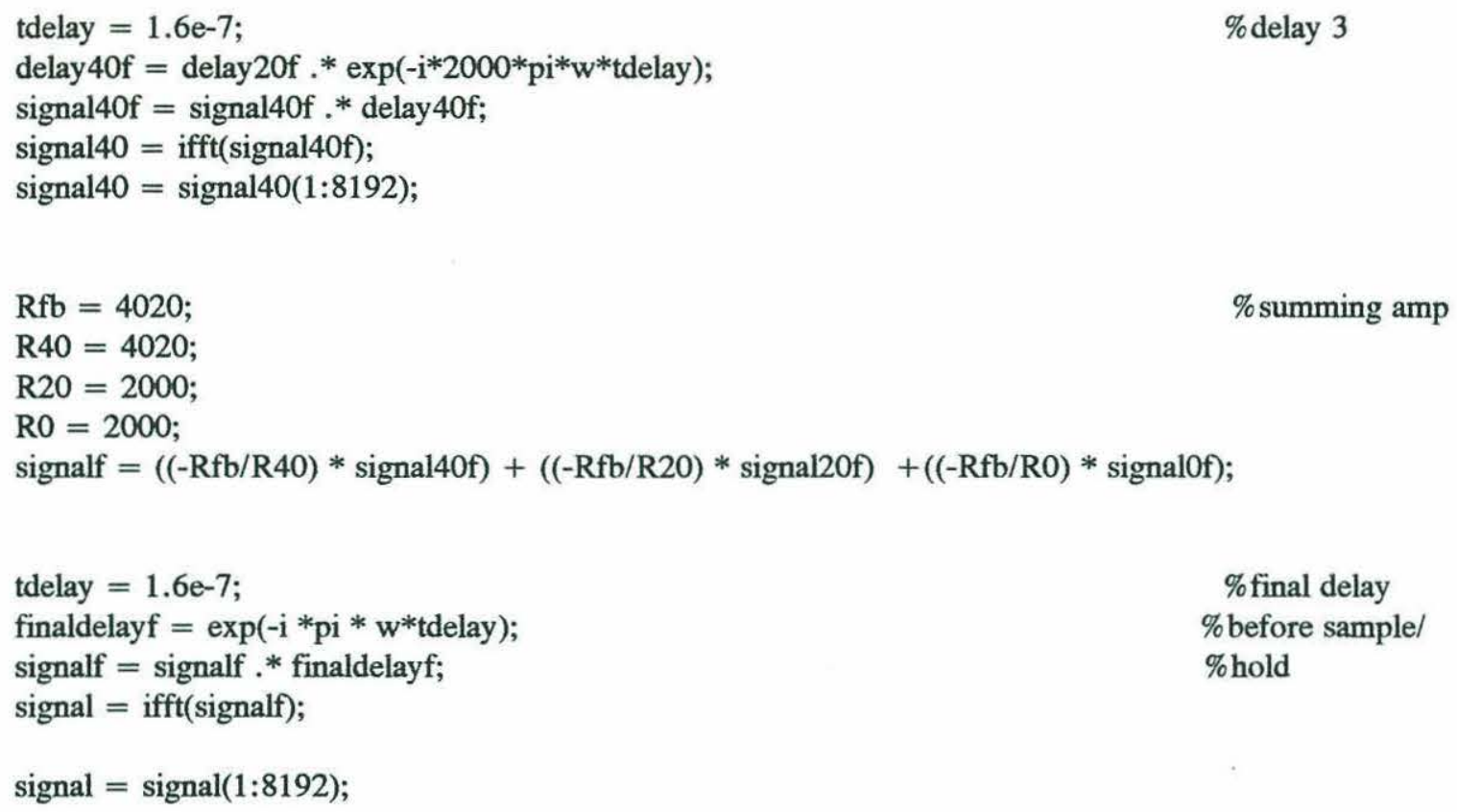

$\%$ final delay \% before sample/ $\%$ hold 


\section{References}

[1] Stewart, W.K., "Multisensor Modeling Underwater with Uncertain Information," Doctoral Dissertation, MIT/WHOI Joint Program in Oceanography and Oceanographic Engineering, Woods Hole, Massachusetts, September 1988.

[2] Denbigh, P.N., "Stereoscopic Side-Scan Sonar," Acoustic Letters, Vol 2, 1978, Pages $108-112$.

[3] Denbigh, P.N., "Swath Bathymetry: Principles of Operation and an Analysis of Errors," IEEE Journal of Oceanic Engineering, Vol 14, October 1989, Pages 289 298.

[4] Chesterman, W.D., J.M.P. St. Quinton, Y. Chan, and H.R. Matthews, "Acoustic Surveys of the Sea Floor Near Hong Kong", International Hydrographic Review, Vol 44, 1967, Pages 35 - 54.

[5] Heaton, M.J.P. and W.G. Haslett, "Interpretation of Lloyd Mirror in Side Scan Sonar," Proceedings of the Society for Underwater Technology, Vol 1, 1971, Pages 24 -38 .

[6] Stubbs, A.R., B. S. McCartney, and J.G. Legg, "Telesounding, A Method of Wide Swathe Depth Measurement," International Hydrographic Review, Vol 51, 1974, Pages 23 - 59. 
[7] Stewart, W. K., "Three-Dimensional Modeling of Seafloor Backscatter from Sidescan Sonar for Autonomous Classification and Navigation," Proc. Symposium on Unmanned Untethered Submersible Technology, June 1989.

[8] Knight, W.C., R.G. Pridham, and S.M. Kay, "Digital Signal Processing for Sonar," Proceedings of the IEEE, Vol 69, November 1981, Pages 1451 - 1505.

[9] Tucker, D.G. and B.K. Gazey, Applied Underwater Acoustics, Pergamon Press, Oxford, 1966.

[10] Sears, F.W., M.W. Zemansky, and H.D. Young, University Physics, AddisonWesley, Reading, Massachusetts, 1976.

[11] Dyer, I., 13.851 Lecture Notes. Massachusetts Institute of Technology, 1990.

[12] Edgerton, H.E., Sonar Images, Prentice - Hall, Englewood Cliffs, New Jersey, 1986.

[13] Siebert, W.M., Circuits, Signals, and Systems, MIT Press, Cambridge, Massachusetts, 1986.

[14] Horowitz, P. and W. Hill, The Art Of Electronics, Cambridge University Press, Cambridge, 1980.

[15] Applied Physics Laboratory, Transducer Group, Technical Data Sheet, University of Washington. 
[16] Klepsvik, J.O., "Topo-SSS: Real - Time Bathymetric Mapping With A Side-Scan Sonar", Proc. of HYDRO '84 - National Ocean Service Hydrographic Conference, April 1984, Pages 42 - 49.

[17] Kinsler, L.E. and A.R. Frey, Fundamentals of Acoustics, John Wiley \& Sons, New York, New York, 1962.

[18] Oppenheim, A.V. and R.W. Schafer, Discrete - Time Signal Processing, Prentice Hall, Englewood Cliffs, New Jersey, 1989.

[19] Smith, R.J. Circuits, Devices and Systems, John Wiley \& Sons, New York, New York, 1966. 